\title{
Occurrence, mating strategy, and pathogenicity of members of Nectriaceae in Central Appalachia
}

\author{
Cameron Michael Stauder \\ West Virginia University, cmstauder@mix.wvu.edu
}

Follow this and additional works at: https://researchrepository.wvu.edu/etd

Part of the Plant Pathology Commons

\section{Recommended Citation}

Stauder, Cameron Michael, "Occurrence, mating strategy, and pathogenicity of members of Nectriaceae in Central Appalachia" (2020). Graduate Theses, Dissertations, and Problem Reports. 7608.

https://researchrepository.wvu.edu/etd/7608

This Dissertation is protected by copyright and/or related rights. It has been brought to you by the The Research Repository @ WVU with permission from the rights-holder(s). You are free to use this Dissertation in any way that is permitted by the copyright and related rights legislation that applies to your use. For other uses you must obtain permission from the rights-holder(s) directly, unless additional rights are indicated by a Creative Commons license in the record and/ or on the work itself. This Dissertation has been accepted for inclusion in WVU Graduate Theses, Dissertations, and Problem Reports collection by an authorized administrator of The Research Repository @ WVU.

For more information, please contact researchrepository@mail.wvu.edu. 
Graduate Theses, Dissertations, and Problem Reports

2020

Occurrence, mating strategy, and pathogenicity of members of Nectriaceae in Central Appalachia

Cameron Michael Stauder

Follow this and additional works at: https://researchrepository.wvu.edu/etd

Part of the Plant Pathology Commons 
Occurrence, mating strategy, and pathogenicity of members of Nectriaceae in Central Appalachia

Cameron Michael Stauder

Dissertation submitted to the Davis College of Agriculture, Natural Resources and Design at West Virginia University in partial fulfillment of the requirements for the degree of

\author{
Doctor of Philosophy \\ in \\ Plant and Soil Sciences \\ Matthew Kasson, Ph.D., Chair \\ William MacDonald, Ph.D. \\ Daniel Panaccione, Ph.D. \\ Jeffrey Garnas, Ph.D.
}

Division of Plant and Soil Sciences

Morgantown, West Virginia
2020

Keywords: beech bark disease, target canker, Neonectria, Corinectria, mating type

Copyright 2020 Cameron Stauder 


\section{ABSTRACT \\ Occurrence, mating strategy, and pathogenicity of members of Nectriaceae in Central Appalachia}

\section{Cameron M. Stauder}

Members of the Nectriaceae occupy many ecological niches including dominant canker pathogens, such as Neonectria ditissima and $N$. faginata. These two pathogens contribute to the decline of American beech (Fagus grandifolia) forests across the Appalachian Mountains due to beech bark disease (BBD). Interestingly, N. ditissima represents a well-known canker pathogen many hardwood species, while $N$. faginata has not been observed outside of BBD. Additionally, N. faginata occurs at higher incidences than $N$. ditissima in BBD stands. Nectriaceae in Central Appalachia were surveyed as to further characterize the diversity and possibly identify a non-beech host of $N$. faginata. This resulted in the recovery of ten nectriaceous species from twelve tree species. Neonectria faginata only occurred on BBD trees. Neonectria ditissima was recovered eight tree species including Acer spicatum, Ilex mucronata, and Sorbus americana. Fusarium babinda was often recovered from BBD trees, but its role in BBD remains unclear. Corinectria gaudineerii sp. nov. was recovered from Picea rubens and Neonectria magnoliae comb. nov. from cankered Liriodendron tulipifera and Magnolia fraseri. The pathogenicity of $N$. magnoliae was confirmed, but the pathogenicity of $C$. gaudineerii was less apparent. Heterothallism for $N$. ditissima, $N$. faginata, and a number of other Nectriaceae was confirmed using molecular data and in vitro assays. This was important different mating strategies might explain differences in the ecology of $N$. faginata and $N$. ditissima. Together, these results demonstrate the diversity of 
Nectriaceae in eastern North America and their mating strategies as to further our understanding of dominant diseases affecting Appalachian forests. 


\section{ACKNOWLEDGEMENTS}

There are many people to acknowledge for the work completed within this dissertation.

First and foremost, I want to thank my wife Laura for being my constant companion and support through these years. Thank you for believing in me even when I didn't believe in myself. Second, l'd like to thank my daughter Hazel for reminding me of what is important every single morning with as little as a smile. Dr. Matt Kasson, I could never thank you enough. We've accomplished a lot together over these years, and I couldn't be more thankful for the support and friendship you've given me along the way. I hope we continue to stay in touch and even continue to collaborate on projects that employ our well-trained "peritheci-eyes". I hope we do eventually find that elusive non-beech host of N. faginata. I would like to also thank Dr. Bill MacDonald for placing his trust in me from the start and for all his support. Mark Double, thank you for always having an open door and being willing to support, not just me, but all graduate students of SAS. Dr. Dan Panaccione thank you for also always having an open door and for teaching me pretty much everything I know about fungi. A big thanks to all of the Kasson lab for their friendships and support over the years. I never forget the memories we've shared. l'd like to thank Jeff Garnas for agreeing to serve on my committee from afar and for his support of my research along the way. Finally, l'd like to thank Nicole Utano, Braley Burke and Amy Metheny for their many hours helping me to process endless numbers of perithecia. This work would not have happened without them. 


\section{TABLE OF CONTENTS}

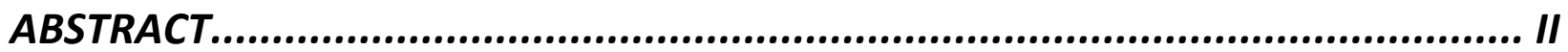

ACKNOWLEDGEMENTS .................................................................... IV

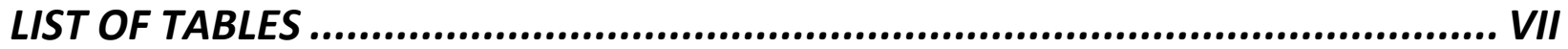

LIST OF FIGURES ................................................................................ VII

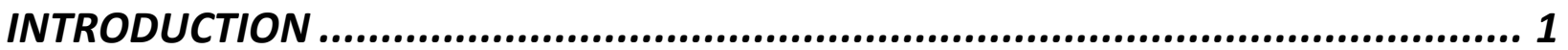

Literature Cited .......................................................................................................................... 5

CHAPTER 1: Resolving host and species boundaries for perithecia-producing nectriaceous fungi across the central Appalachian Mountains......................... 7

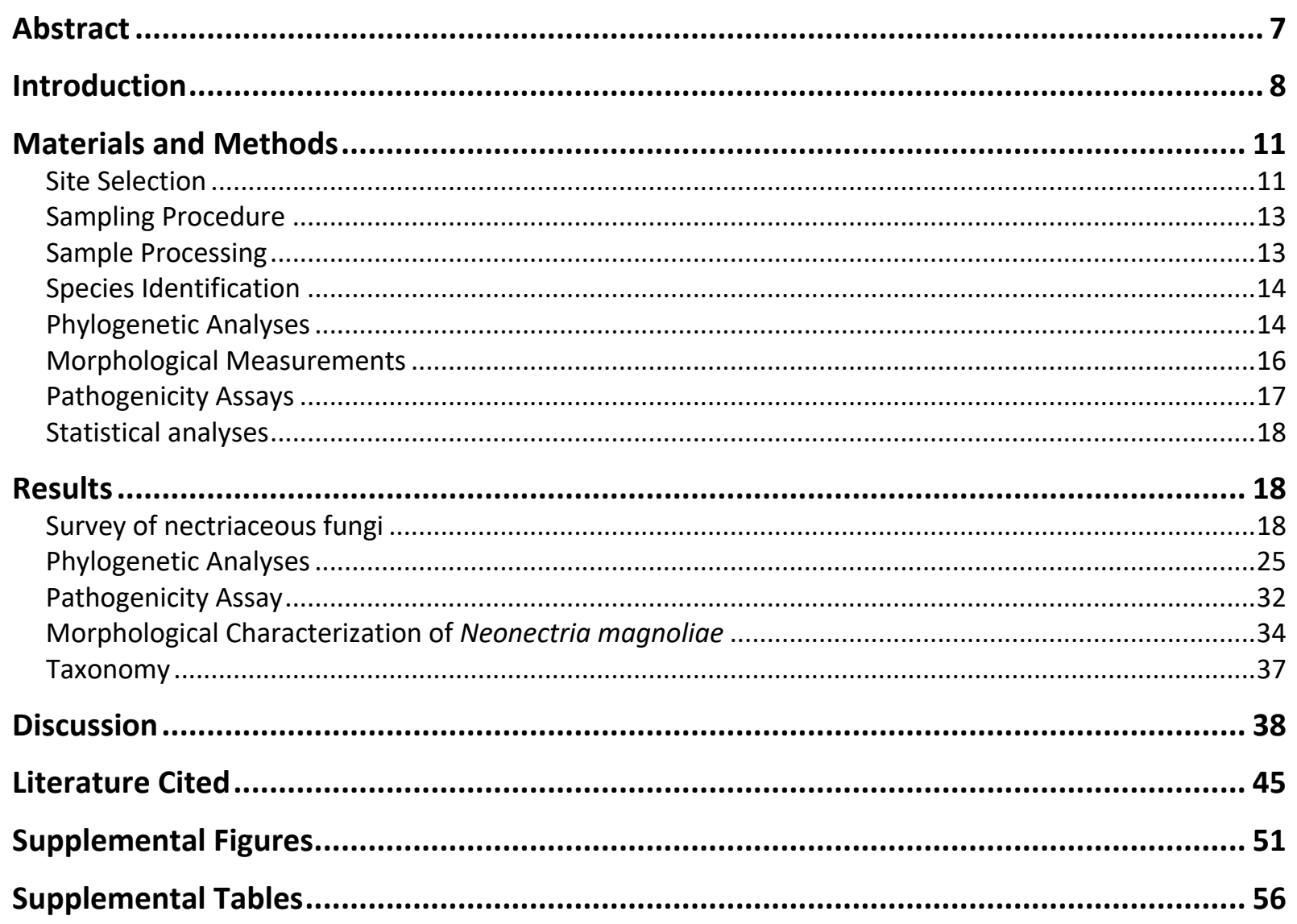

CHAPTER 2: Characterization of mating type genes in heterothallic Neonectria species with emphasis on $N$. coccinea, $N$. ditissima, and $N$. faginata .................61

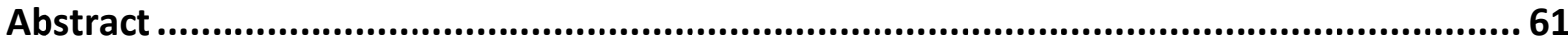

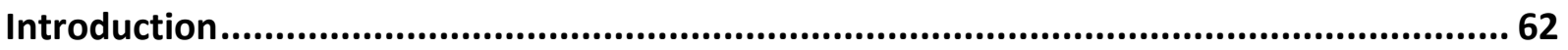

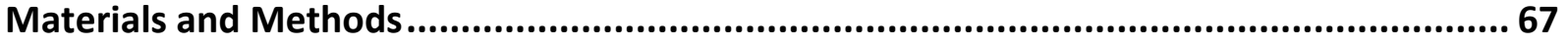

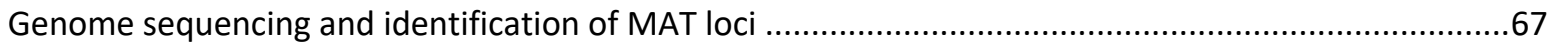


Characterizing the structure of MAT loci.

Species-specific MAT 1-1-1 and MAT 1-2-1 primer design and PCR amplification...............................69

Genus-level MAT 1-1-1 and MAT 1-2-1 primer design and PCR amplification .....................................74

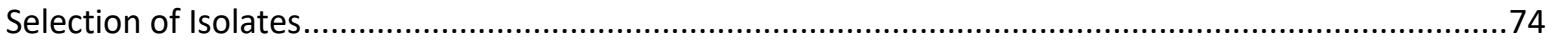

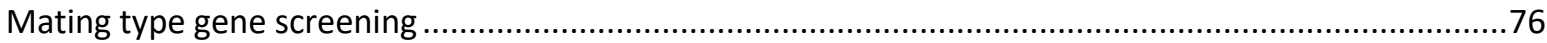

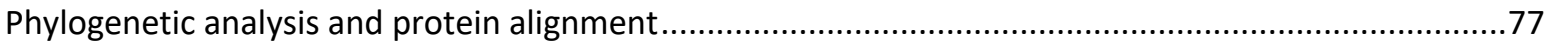

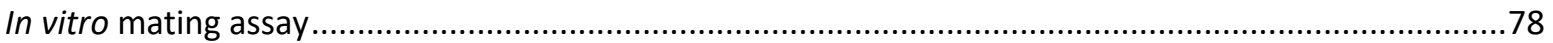

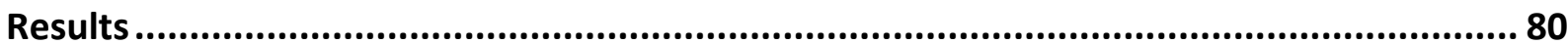

Identification and structure of MAT loci in $N$. ditissima and $N$. faginata ............................................80

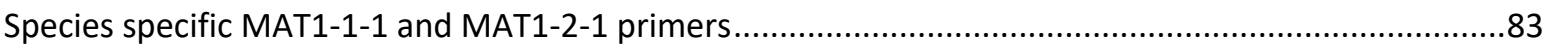

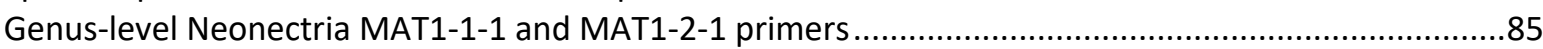

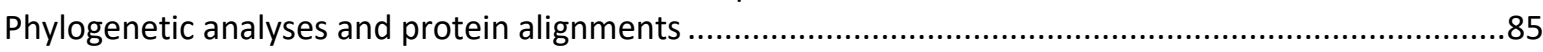

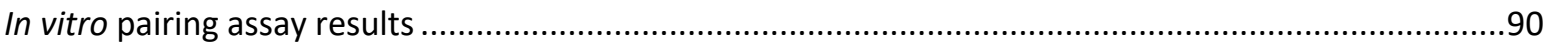

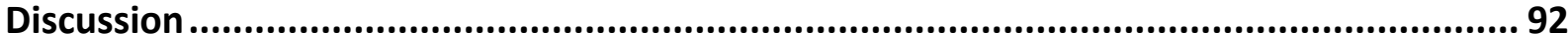

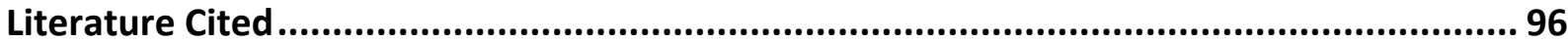

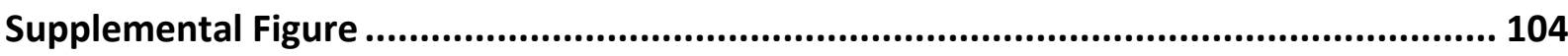

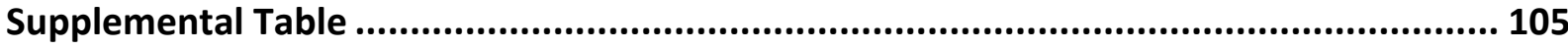

CHAPTER 3: Corinectria gaudineerii sp. nov., a novel nectriaceous fungus associated with high elevation Picea rubens in the Appalachian Mountains ...106

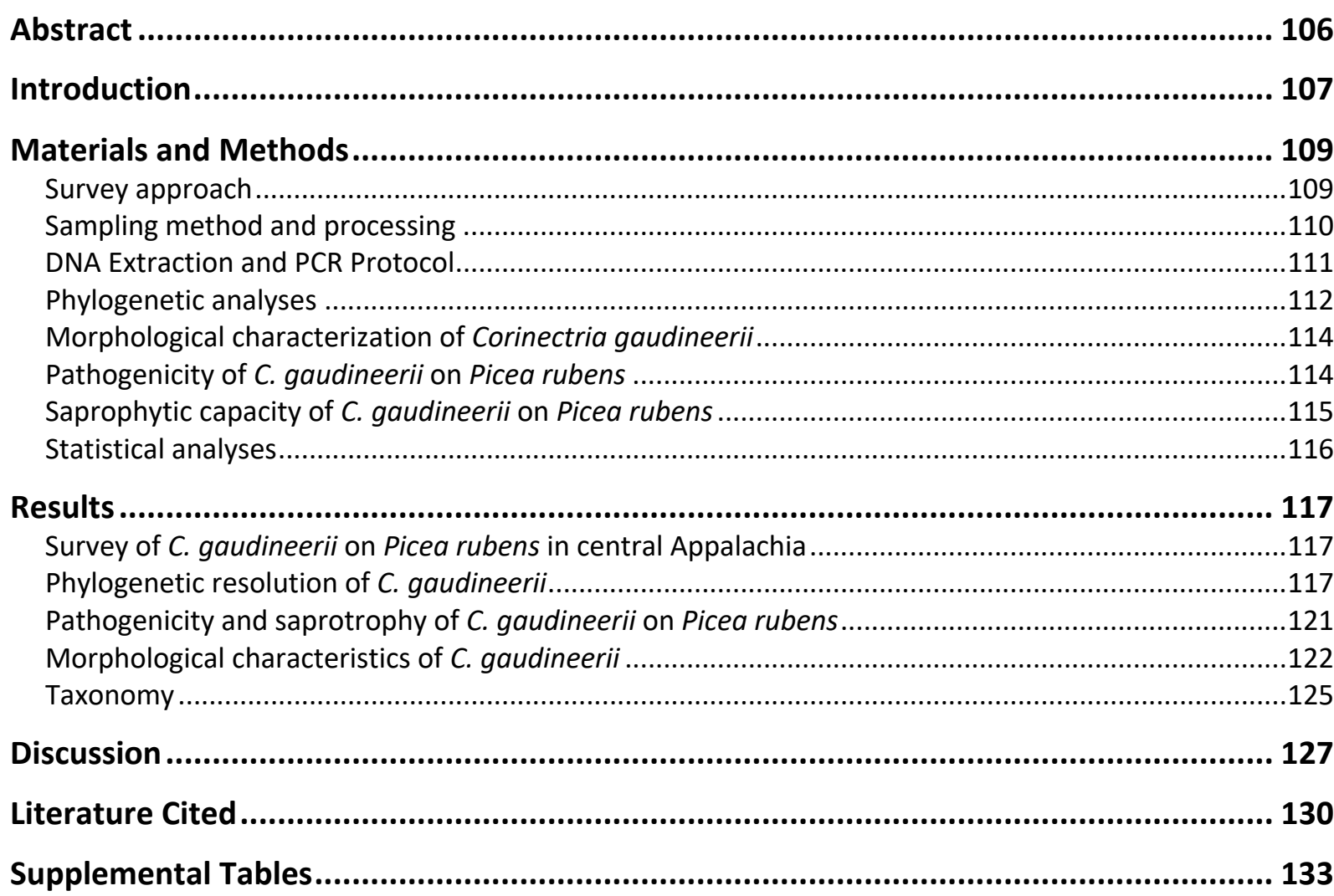




\section{LIST OF FIGURES}

\section{CHAPTER 1}

Figure 1: Sampling locations for members of Nectriaceae in the central Appalachian Mountains. Sites with confirmed beech bark disease (BBD) are designated by filled circles, sites lacking BBD are open circles, and counties where BBD has been previously reported are filled. BBD was first discovered in WV at site GK in 1981 (Mielke et al. 1982, Mielke and Houston, 1983). BBD distribution is based on a county level database (Blackburn, 2018).

Figure 2: Example Neonectria ditissima canker photos for the following host species: A) mountain holly (Ilex mucronate at SK), B) American beech (Fagus grandifolia at GK), C) mountain maple (Acer spicatum at MM), D) black birch (B. lenta at UF), E) mountain ash (Sorbus Americana at WT), F) striped maple ( $A$. pensylvanicum at SK)

Figure 3: Diversity of Nectriaceae recovered across the central Appalachian Mountains on natural substrate and in culture: A) Neonectria ditissima (shown on striped maple at SK), B) N. faginata (on American beech at BG), C) N. neomacrospora (on Fraser fir at MR), D) Corinectria sp. (on red spruce at GK), E) Thryonectria balsamea (on balsam fir at MON), F) Thelonectria veuillotiana (on mountain maple at MM), G) Bionectria ochroleuca (on American beech at SM), and H) Cosmospora obscura (on mountain ash at MM).

Figure 4: Four-gene (ITS, BTUB, ACTIN, EF1) concatenated phylogeny tree of Neonectria spp., Corinectria spp. and outgroups. Topology and branch lengths are from the $\mathrm{ML}$ analysis. For each node supported in the ML analysis, bootstrap support and posterior probabilities are indicated $(\mathrm{ML} / \mathrm{BI})$.

Figure 5: Pathogenicity results and representative photos for each host species tested: $A$ ) striped maple (Acer pensylvanicum), B) yellow birch (Betula alleghaniensis), C) black birch (B. lenta), D) American beech (Fagus grandifolia), and E) tulip poplar (Liriodendron tulipifera). Letters designate significant differences at $p<0.05$. All sample sizes are 6 trees, except for American beech

Figure 6: Neonectria magnoliae: A) Example canker on Liriodendron tulipifera with perennial target canker morphology; B) Example canker on L. tulipifera exhibiting less distinct canker morphology; C) Example canker on Magnolia fraseri; D) N. magnoliae perithecia erupting from L. tulipfera bark tissues; E) Asci containing eight ascospores each; F) Ascospores; G) Ornamented surface of ascospores; H) Top and bottom of 7-day old culture photos on PDA; I) Creamy microconidial sporodochia and aerial conidiophores; J) Macroconidia with four septations; K) Microconidia of varying lengths. Scale bars $=250 \mu \mathrm{m}$ for panel D; $20 \mu \mathrm{m}$ for panels $\mathrm{E}, \mathrm{K} ; 10 \mu \mathrm{m}$ for panels $\mathrm{F}, \mathrm{G}, \mathrm{J}$;

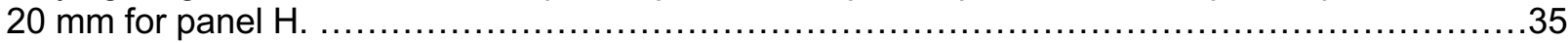

Figure 7: Neonectria magnoliae holotype (BPI 552527 [No. 64184]) submitted by Lohman and Heptig in 1934. Sample consists of N. magnoliae fruiting on bark of tulip poplar (Liriodendron tulipifera) collected in Richwood, West Virginia, USA. Photos provided by Lisa A Castlebury, Ph.D., Acting Research Leader and Collections Director, USDA ARS MNGDBL. ........................36

Supplemental Figure 1: Morphological characteristics of Fusarium babinda: A) F. babinda sporulation on Neonectria sp. perithecia; B) 7-day old culture on PDA; B) sporodochia bearing macro- and micro-conidia; C) macroconidia; D) microconidia.

Supplemental Figures 2 - 5: Single-gene (BTUB, EF1, ITS, LSU) phylogenetic tree of Neonectria spp., Corinectria spp. and outgroups. Topology and branch lengths are from the ML analysis. For 
each node supported in the ML analysis, bootstrap support and posterior probabilities are indicated

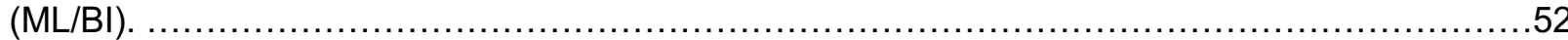

\section{CHAPTER 2}

Figure 1: In vitro mating assay for Neonectria spp. and related fungi: A) Approximately $5 \mathrm{~mm} \times 5 \mathrm{~mm}$ colonized plugs of confirmed MAT1-1 and/or MAT1-2 strains placed on either side of a sterile wooden toothpick placed upon GYE media; B) Perithecia exuding ascospores on toothpick after approximately 12 weeks; C) Perithecia squash mount showing ascospores within asci. Neonectria magnoliae shown in figure. Individual scale bars are included in each panel.

Figure 2: Structure of the MAT1-1 and MAT1-2 loci of the heterothallic fungi: Neonectria ditissima, Neonectria faginata, and N. coccinea (MAT1-2 only). Arrows above the genes indicate the 5' - 3' orientation. Coding sequence lengths are included below each gene identifier. For MAT1-1-1 and MAT1-2-1 genes, introns are represented by vertical black lines, and approximate primer binding locations are illustrated for each primer pair below the gene illustration. The approximate amplicon size for each primer pair is shown below the primer binding locations and is shaded based on each primer pair. All distances and sizes are estimations and not drawn to scale.

Figure 3: Phylogenetic relationships of Neonectria spp. and related fungi based on MAT1-1-1 gene sequence data. The phylogeny was inferred using a Maximum Likelihood analysis based on the Kimura 2-parameter model with gamma distribution $(K 2+G)$ and 1000 bootstrap replicates. Bootstrap values $>70 \%$ are given at the nodes. Branch lengths represent the number of substitutions per site. Outgroup includes Ophiocordyceps xeufengensis and Ustilaginoidea virens. .86

Figure 4: Phylogenetic relationships of Neonectria spp. and related fungi based on MAT1-2-1 gene sequence data. The phylogeny was inferred using a Maximum Likelihood analysis based on the Kimura 2-parameter model with gamma distribution $(K 2+G)$ and 1000 bootstrap replicates. Bootstrap values $>70 \%$ are given at the nodes. Branch lengths represent the number of substitutions per site. Outgroup includes Ophiocordyceps xeufengensis and Ustilaginoidea virens. .87

Figure 5: MAT1-1-1 and MAT1-2-1 amino acid alignments including a selection of study fungal species. Name abbreviations are as follows: $\mathrm{Nef}=$ Neonectria faginata; Nec = Neonectria coccinea; Nep = Neonectria punicea; Necm = Nectria magnoliae; Ned = Neonectria ditissima; Nen = Neonectria neomacrospora; Neh = Neonectria hederae; Cof = Corinectria fuckeliana; Coaf = Corinectria aff. fuckeliana; Fub = Fusarium babinda; Fuo = Fusarium oxysporum; Usv = Ustilaginoidea virens; Opx = Ophiocordyceps xuefengensis; Cons = consensus. Uppercase letters within the consensus sequence represent positions with identical amino acids. Lower case letters represent positions with similar amino acids. Black shading represents conserved amino acids among $50 \%$ or more species. Grey shading represents shared amino acids with shared characteristics/properties among species.

Supplemental Figure 1: Electrophoresis gel photo demonstrating specific amplification of MAT1-1-1 and MAT1-2-1 by the genus-level primer pair (NeoM1f/r and NeoM2f/r) for both $N$. ditissima and N. faginata. 104

\section{CHAPTER 3}

Figure 1: Sampling locations for members of Nectriaceae on red spruce in WV and VA. These include Mount Rogers (MR) and Whitetop Mountain (WT) of the Jefferson National Forest in VA and areas near Cranberry Wilderness (CR) and Gaudineer Knob (GK) of the Monongahela National Forest in WV. Shaded areas represent the natural range of red spruce 
Figure 2: Concatenated sequence (ACT, BTUB, EF1, ITS, LSU) phylogenetic tree of Corinectria spp. and Neonectria spp. outgroups. Topology and branch lengths are from the ML analysis. For each node supported in the ML analysis, bootstrap support and posterior probabilities are indicated $(\mathrm{ML} / \mathrm{BI})$

Figure 3: Single-gene (ACT, BTUB, EF1, ITS, LSU) phylogenetic trees of Corinectria spp. and Neonectria spp. outgroups. Topology and branch lengths are from the $\mathrm{ML}$ analysis. For each node supported in the ML analysis, bootstrap support and posterior probabilities are indicated ....

Figure 4: A: Live red spruce stem assay results for inoculations of Corinectria gaudineerii from two separate locations (WV and VA) and a negative control. B: Excised red spruce stem assay for inoculations with $C$. gaudineerii, Neonectria ditissima, $N$. faginata, and negative control. Letters designate significant differences at $p<0.05$.

Figure 5: Corinectria gaudineerii: A) Example of abundant fruiting on Picea rubens; B) C. gaudineerii perithecia erupting from $P$. rubens bark tissues; C) Ascus containing eight ascospores; D) Ascospore; E) Ornamented surface of ascospore; F) Top of 10-day old culture photos on PDA; G) Bottom of culture; H) Microconidial sporodochia underneath aerial conidiophores; I) Microconidia of varying lengths; J) Close-up of microconidia; Scale bars $=250 \mu \mathrm{m}$ for panel D; $20 \mu \mathrm{m}$ for panels $\mathrm{E}$, $\mathrm{K} ; 10 \mu \mathrm{m}$ for panels $\mathrm{F}, \mathrm{G}, \mathrm{J} ; 20 \mathrm{~mm}$ for panel $\mathrm{H}$. .124

\section{LIST OF TABLES}

\section{CHAPTER 1}

Table 1: Survey sample collection summary by host tree species. Values under each fungal species represent the number of sampled perithecia yielding an isolate of that fungus. In total, 1,605 perithecia yielded nine fungal species from ten host tree species at 17 geographically separated sites.

Table 2: Isolate identification by BLASTn searches using ITS sequences derived from selected representatives of dominant morphologies. Percent identity values represent the lowest identity value among the sequences for each fungal species.

Table 3: Accession table for sequences used in the phylogenetic analyses of this study. Sequences were either acquired from NCBI or generated as part of this study. Host species and location are also provided where available.

Supplemental Table 1: Locations of sampling sites (including areas within these sites if applicable). The presence of BBD in each site is noted. Additionally, the general coordinates of the sampling locations are provided.

Supplemental Table 2: Primer sequences, PCR parameters, and sources for each primer pair used to generate sequence data.

Supplemental Table 3: All BLASTn results from representative isolates recovered in this survey. The accession number, query coverage, and percent identity are provided for the top hit for each sequence. 
Supplemental Table 4: Recovery of Fusarium babinda isolates from bark tissue samples collected from asymptomatic American beech trees (shaded grey) or American beech trees with signs or symptoms associated with beech bark disease. Values represent the number of bark samples yielding Fusarium babinda at the designated sampling location.

\section{CHAPTER 2}

Table 1: Identity, source location, and host substrate of fungal isolates used in this study. GenBank accession numbers for derived and submitted sequences are included.

Table 2: MAT1-1-1 and MAT1-2-1 primers designed in this study, including the corresponding amplicon sizes and PCR protocols.

Table 3: Summary PCR results for each included species and primer pair with '+' denoting successful amplification of the target sequence and "*" indicating non-target amplification when primer pair is applied to an isolate of the opposite mating type.

Table 4: In vitro pairing assay results for $N$. ditissima and $N$. faginata. PCR confirmed MAT identities are included for each isolate. Three pairings were completed for each isolate set. Result format $=$ number of positive results out of the three total pairings. Results categories include the following: 1. Production of perithecia; 2. Presence of ascospores within perithecia 3. Viability of ascospores from ascospore suspension plates; 4 . Production of perithecia on ascospore suspension plates used for viability confirmation.

Supplemental Table 1: Non-target amplification identities for primer $x$ species pairings: MAT identities are given with the species names. The approximate product sized expected for specific amplification of the target MAT gene are provided. For instances of multiple non-target amplicons among tested species, the lowest sequence similarity value is given. Non-target protein blastx results with the greatest percent sequence coverage are provided, including query coverage, percent identity, and $\mathrm{NCBI}$ accession numbers.

\section{CHAPTER 3}

Table 1: Accession table for sequences used in this study. Sequences were either generated here or acquired from NCBI. Host species and location are also provided where available.

Supplemental Table 1: Locations of survey sites. The presence of Corinectria gaudineerii is noted for each site. Additionally, the general coordinates of the sampling locations are provided. Samples were only collected within permitted areas.

Supplemental Table 2: Primer sequences, PCR protocols, and sources for each primer pair used to generate sequence data within this study. 


\section{INTRODUCTION}

Members of the fungal family Nectriaceae occupy a number of diverse ecological niches, including mycoparasites, entomopathogens, and phytopathogens. Many species across multiple genera have been implicated in causing annual and perennial cankers on diverse woody plant hosts. Among these are generalist canker pathogens capable of infecting multiple host species, such as Neonectria ditissima ([Tul. \& C. Tul.] Samuels \& Rossman) (Lohman and Watson 1943; Spaulding et al. 1936; Booth 1967), and apparent specialists only known to infect a single host species, such as N. faginata ([Lohman, Watson, \& Ayres] Castl. \& Watson) (Castlebury et al., 2006). Interestingly, both of these are implicated in a disease known as beech bark disease (BBD).

$\mathrm{BBD}$ is a disease complex involving both insect and fungal agents of American beech (Fagus grandifolia) trees throughout eastern North America and European beech (Fagus sylvatica) in Europe (Hewitt, 1914; Erhlich, 1934; Cale et al., 2017). In both locations, the disease requires prior infestation by an exotic scale insect (Cryptococcus fagisuga) (Erhlich, 1934). Infestation by this scale insect predisposes the host tissues to invasion by one or more closely related canker fungi: Neonectria ditissima, $N$. faginata, and N. coccinea ([Pers.] Rossman and Samuels) (Houston 1994b; Thomsen et al. 1949). Neonectria ditissima (formerly $N$. galligena Bres.) infects beech in both North America and Europe, while $N$. faginata (formerly $N$. coccinea var. faginata) appears restricted to North America and N. coccinea to Europe (Thomsen et al. 1949; Castlebury et al. 2006).

The origin and ecological niche of Neonectria faginata outside of BBD is unknown. Castlebury et al. (2006) suggested $N$. faginata to be a native fungus based on 
an expected level of genetic diversity present among fungal strains isolated across the distribution of BBD. Additionally, surveys conducted throughout the distribution of BBD report a dominant presence of $N$. faginata in comparison to $N$. ditissima (Houston, 1994b; Kasson and Livingston, 2009). This dominance appears to be gained over time as $N$. ditissima appears dominant early in the progression of BBD and is supplanted by N. faginata as the dominant pathogen (Houston 1994b). The progression of $N$. faginata across the landscape following the spread of the predisposing scale insect (C. fagisuga) may explain this supplantation as the inoculum potential of the native $N$. ditissima would be initially greater if already established on co-occurring hardwood species. Kasson and Livingston (2009) provided evidence supporting this theory as higher densities of nonbeech, $N$. ditissima-susceptible species were correlated with higher abundances of $N$. ditissima associated with BBD trees. A number of potentially advantageous characteristics of $N$. faginata have yet to be properly investigated, including its virulence and fecundity on American beech in comparison to N. ditissima.

Prior to the advent of molecular phylogenetics, studies relied on the morphological identification of Neonectria fungi to species, and as such, the misidentification of $N$. faginata on another host substrate is plausible. $N$. faginata was first morphologically identified as a variety of $N$. coccinea (previously Nectria coccinea) as Nectria coccinea var. faginata (Booth, 1977), but later was determined to be a distinct species based on phylogenetic analyses (Castlebury et al., 2006). Other Neonectria species native to eastern North America exhibit a mosaic of morphological attributes, some of which overlap with N. faginata (Lohman and Watson, 1943). These factors may have led to an underrepresentation of the overall diversity of Nectriaceae 
through the misidentification of species when depending on morphological comparisons. Additionally, given the plausibility of a previous misidentification of N. faginata on a nonbeech host, additional studies are warranted as such a discovery could provide valuable insight into understanding the ecology of $N$. faginata, including its role in the BBD pathosystem.

Members of Nectriaceae, including $N$. ditissima and $N$ faginata, are often recognized by the production of brightly colored perithecia. These globose fruiting bodies bear ascospores, the products of sexual mating. While perithecia are often products of sexual reproduction between two distinct thalli (heterothallism), a self-fertile condition in fungi known as homothallism can also yield these structures through selfmating (Alexopolous et al. 1996). Both homothallism and heterothallism have been reported in N. ditissima (El-Gholl et. al, 1986; Krüger, 1973), and heterothallism has been reported for $N$. faginata (Cotter and Blanchard, 1978). While the role of ascospores in BBD is limited, there is evidence that ascospore production may affect disease progression. For example, ascospores of $N$. ditissima have been demonstrated as the dominant spore type disseminating from yellow birch infections (Lortie and Kuntz, 1963). BBD progression also may depend on ascospore production and dissemination, and thus, the observed supplantation of $N$. ditissima by $N$. faginata may be in part due to mating strategy as homothallic fungi may more readily produce and disseminate ascospores in nature.

The research presented in this dissertation is intended to provide additional insight into the ecology of $N$. faginata and $N$. ditissima, but these findings are not limited to these two fungi and their associated diseases. In the first chapter, perithecia-forming 
nectriaceous fungi occurring on beech and non-beech trees present in forested stands with BBD are surveyed to identify possible alternate substrates of $N$. faginata. This is critical to understanding the ecology of $N$. faginata within and outside of the BBD complex. The second chapter molecularly characterizes the MAT loci of $N$. ditissima and $N$. faginata as to determine the mating strategy. This is important as the progression of BBD may be dependent on the production and dissemination of ascospores and mating strategy could inherently influence this progression. Within Chapters 2 and 3 previously undescribed Nectriaceae or plant $\mathrm{x}$ host interactions occurring in eastern North America are investigated. Together these investigations may provide a greater understanding of the ecology of nectriaceous fungi within and outside of BBD. 


\section{Literature Cited}

Alexopolous CJ, Mims CW, Blackwell M. 1996. Introductory Mycology, 4th ed.

John Wiley \& Sons, Inc, New York.

Booth C. 1967. Nectria galligena. CMI Descriptions of Pathogenic Fungi and Bacteria $147: 1-2$

Booth, C., 1977. Nectria coccinea var. faginata. C.M.I. Descriptions of Pathogenic Fungi and Bacteria, No. 533 ref.8.

Cale JA, Garrison-Johnston MT, Teale SA, Castello JD. 2017.Beech bark disease in North America: Over a century of research revisited. Forest Ecology and Management 394: 86-103.

Castlebury LA, Rossman AY, Hyten AS. 2006. Phylogenetic relationships of Neonectria/Cylindrocarpon on Fagus in North America. Canadian Journal of Botany 84: 1417-1433.

Cotter HVT, Blanchard RO. 1978. Heterothallism in Nectria coccinea var. Faginata. Mycologia 70:697-700.

Ehrlich, J. 1934. The beech bark disease, a Nectria disease of Fagus, following Cryptococcus fagi (Baer.). Canadian Journal of Research 10: 593-692.

EI-Gholl NE, Barnard EL, Schroeder RA. 1986. Homothallism in Nectria galligena. Canadian Journal of Botany. 64: 902-903.

Hewitt CG. 1914. Note on the occurrence of the felted beech coccus Cryptococcus fagi (Baerens) Dougl. in Nova Scotia. The Canadian Entomologist 46:15-16. Houston DR. 1994a. Temporal and spatial shift within the Nectria pathogen complex 
associated with beech bark disease of Fagus grandifolia. Canadian Journal of Forest Research 24:960-968.

Houston DR. 1994b. Major new tree disease epidemics: beech bark disease. Annual Review of Phytopathology 32:75-87.

Kasson MT, Livingston WH. 2009. Spatial distribution of Neonectria species associated with beech bark disease in northern Maine. Mycologia 101:190-195.

Krüger J. 1973. Zur genetik von Nectria galligena Bres. Journal of Phytopathology 79: 320-342.

Lohman ML, Watson AJ. 1943. Identity and host relations of Nectria species associated with diseases of hardwoods in the Eastern States. Lloydia 6: 77-108.

Lortie, M., Kuntz, J. E. 1963. Ascospore discharge and conidium release by Nectria galligena Bres. under field and laboratory conditions. Canadian Journal of Botany. 41: 1203-1210.

Spaulding P, Grant TJ, Ayers TT. 1936. Investigations of Nectria diseases in hardwoods of New England. Journal of Forestry 34:169-179.

Thomsen M, Buchwald NF, Hauberg, P. 1949. Angreb af Cryptococcus fagi, Nectria galligena og andre parasiter paa bog i Danmark 1939-43. Det Forstlige Forsogsvaesen, 18: 97-326. 


\title{
CHAPTER 1: Resolving host and species boundaries for perithecia- producing nectriaceous fungi across the central Appalachian Mountains
}

\begin{abstract}
The Nectriaceae contains numerous canker pathogens. Due to scarcity of ascomata on many hosts, comprehensive surveys are lacking. Here the diversity of peritheciaproducing nectriaceous fungi across the central Appalachians is characterized. Ten species from twelve hosts were recovered including a novel Corinectria sp. from Picea rubens. Neonectria ditissima and $N$. faginata were most abundant and associated with Fagus grandifolia with beech bark disease (BBD). N. ditissima also was recovered from additional cankered hardwoods, including previously unreported Acer spicatum, Ilex mucronata, and Sorbus americana. Cross-pathogenicity inoculations of $N$. ditissima confirmed susceptibility of Acer and Betula spp. Neonectria magnoliae was recovered from cankered Liriodendron tulipifera and Magnolia fraseri and pathogenicity on $L$. tulipifera was confirmed. Fusarium babinda was consistently recovered from beech with $\mathrm{BBD}$, although its role remains unclear. This survey provides a contemporary snapshot of Nectriaceae diversity across the Appalachian Mountains. The following nomenclatural changes are proposed: Neonectria magnoliae comb. nov.
\end{abstract}




\section{Introduction}

Members of the Nectriaceae occupy diverse ecological niches from mycoparasites to phytopathogens, with numerous genera and species implicated in causing annual and perennial cankers on diverse woody plant hosts with varying degrees of host specificity. One such canker disease, beech bark disease (BBD), is a disease complex occurring across the range of American beech (Fagus grandifolia Ehrh.) in North America and European beech (Fagus sylvatica L.) in Europe. The disease requires prior infestation by an exotic scale insect (Cryptococcus fagisuga Lind.), which predisposes the host bark tissues to subsequent invasion by predominantly one of two canker fungi: Neonectria ditissima ([Tul. \& C. Tul.] Samuels \& Rossman) and either N. faginata ([Lohman, Watson, \& Ayres] Castl. \& Watson) on American beech or N. coccinea ([Pers.] Rossman and Samuels) (Houston, 1994b; Thomsen et al., 1949). In North America, BBD was first observed in Halifax, Nova Scotia, Canada around 1890 and presently continues to spread throughout the continuous range of American beech (Hewitt, 1914; Erhlich, 1934; Cale et al., 2017). Other members of Nectriaceae have occasionally been associated with BBD, including Bionectria ochroleuca ([Schwein.] Schroers \& Samuels) and Fusarium spp., but their roles, if any, in BBD are not well understood (Cotter and Blanchard, 1982; Houston et al., 1987; Kasson and Livingston, 2009).

In addition to infecting scale-infested beech, Neonectria ditissima (formerly $N$. galligena) is well-known perennial target canker pathogen on many hardwood tree species often co-occurring in beech forests impacted by BBD (Lohman and Watson, 1943; Spaulding et al., 1936; Booth, 1967). Unlike BBD, which requires predisposition of 
host tissues by beech scale or possibly Xylococculus betulae (Cale et al., 2015), no insect partner has yet been established as a causal factor for $N$. ditissima infection on non-beech hosts. Based on previous pathogenicity studies, $N$. ditissima strains occurring in eastern North America does not exhibit host specificity (Plante and Bernier, 1997), allowing unfettered interactions between inoculum produced from cankers on beech and non-beech hosts. Although this has not been confirmed among all dominant host species co-occurring with beech in BBD areas, namely black birch (Betula lenta L.) and striped maple (Acer pensylvanicum L.), mating barriers do not exist among these strains. This indicates there is likely no host specificity given an outcrossing population of strains occurring on varying host species (Chapter 2). Further investigations are warranted as unknown host specificity and mating barriers could theoretically influence the population dynamics of $N$. ditissima strains participating in the BBD complex by limiting or disrupting gene flow.

In contrast to $N$. ditissima, $N$. faginata (formerly N. coccinea var. faginata) has only been observed causing cankers following $C$. fagisuga infestation on American beech trees (Castlebury et al., 2006), leaving questions regarding its origin and ecological niche, if any, outside of BBD. Other closely related Neonectria species such as N. punicea also occur in eastern North America within northern hardwood forests and exhibit morphological attributes that overlap with $N$. faginata including ascospore size (Booth, 1959; Castlebury et al., 2006), which has historically served as a diagnostic measure in previous studies (Houston, 1994a; Kasson and Livingston, 2009; Lohman and Watson, 1943). As such, previous misidentifications of Neonectria spp. on beech and non-beech hosts are plausible when relying on morphological attributes, possibly 
underestimating diversity (for example see Fig 2 in Kasson and Livingston, 2009). Likewise, the close relationships among Neonectria spp., coupled with the lack of sequence data in repositories such as $\mathrm{NCBI}$, has also presented species identification challenges, especially for rarer species, which further complicates identification. For example, our recent phylogenetic analyses of mating type gene sequences for Nectria magnoliae from Liriodendron tulipifera L. revealed this species formed a genealogically exclusive clade among other Neonectria species that was closely allied with N. faginata (Chapter 2). This supports previous findings by Gräfenhan and colleagues (2011), who showed but did not discuss that $N$. ditissima and $N$. magnoliae were genealogically exclusive. However, earlier work by Castlebury et al. (2006) concluded N. magnoliae was a synonym of $N$. ditissima. These findings emphasize the need for enhanced surveys to recover and phylogenetically resolve cryptic and understudied members of Nectriaceae as well as investigate the potential for cryptic reservoirs of known species including the possibility of $N$. faginata occurring on a non-beech host.

This study sought to provide a greater understanding of the ecology and genetic relationships among $N$. ditissima, $N$. faginata, and allied fungi. The first objective of this study was to survey perithecia-producing members of Nectriaceae in American beech stands to identify possible native reservoirs of $N$. faginata. This is important given the uncertainty surrounding the origin of $N$. faginata and the potential for previous misidentifications of nectriaceous fungi recovered from non-beech hosts. The second objective was to resolve phylogenetic relationships among described and possibly undescribed members of Nectriaceae recovered from forests across the central Appalachian Mountains. This was critical given both the significant undersampling of 
nectriaceous fungi in these regions, the resulting the lack of sequence data, and/or contradictory evidence regarding certain known members of Nectriaceae, such as Nectria magnoliae. A third objective was to test host specificity of $N$. ditissima, $N$. faginata, and N. magnoliae strains isolated from American beech trees and tulip poplar. Together these aims sought to provide contemporary insights into the true diversity, phylogenetic relationships, and ecological niches of perithecia-producing nectriaceous fungi across the central Appalachian Mountains.

\section{Materials and Methods}

\section{Site Selection}

Sampling locations were selected based on previous reports of cankers and/or perithecia production on woody hosts, accessibility, and ability to secure sampling permits. In total, 13 beech bark disease (BBD) and eight non-BBD sampling locations were selected across WV, MD, VA, PA, TN and NC (Figure 1; Supplemental Table 1). Four of the eight non-BBD sites served for sampling nectriaceous fungi from non-beech hosts, while the other four served as sites where beech tissue samples were collected from stands with no history of BBD. No defined sampling areas (i.e. plots) were established, but instead, trees were sampled opportunistically until sampling needs were met based on the sampling procedure described below. Sites GK, MM, and SM had five, two, and four geographically separated discrete sampling areas (Supplemental Table 1). All other sites are represented by a single sampling area.

Three WV sites (BM, GK, FR) were originally confirmed as having BBD at least 35 years prior to the initiation of this study (Mielke et al., 1982, Houston, 1994a). Sites designated BG, DM, and PSF represented more recent BBD outbreaks ( 15 years) as 
determined by various natural resource agency surveys in WV and MD. All other sites were determined to have likely become afflicted with BBD between 5 -to-25 years prior (Morin et al., 2007). Four of seven sites lacking BBD (NB, PM, AR, UW) were selected for asymptomatic beech sampling based on a lack of previous reports, and all trees were visually confirmed to be absent of BBD-associated signs and symptoms prior to sampling.

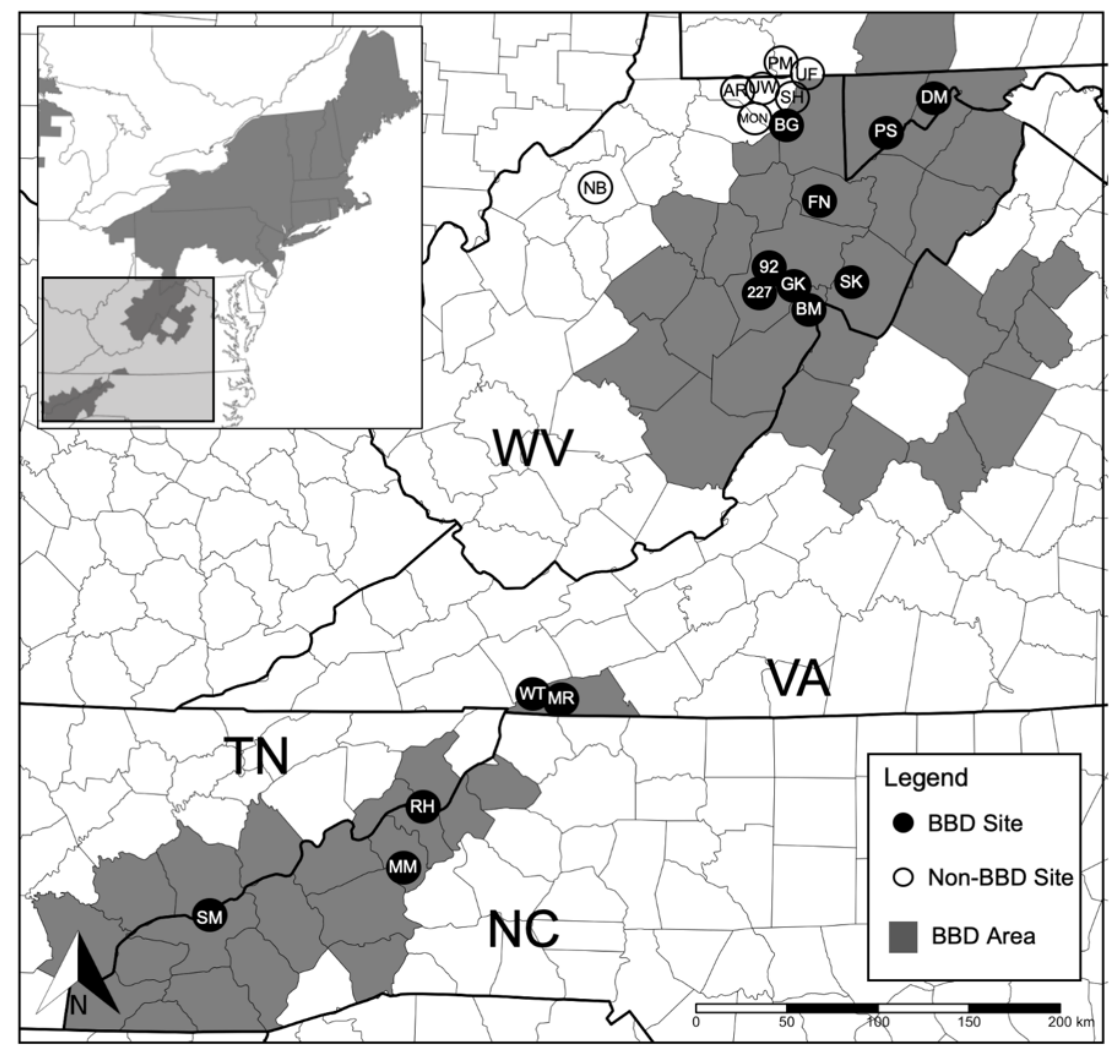

Figure 1: Sampling locations for members of Nectriaceae in the central Appalachian Mountains. Sites with confirmed beech bark disease (BBD) are designated by filled circles, sites lacking BBD are open circles, and counties where BBD has been previously reported are filled. BBD was first discovered in WV at site GK in 1981 (Mielke et al. 1982, Mielke and Houston, 1983). BBD distribution is based on a county level database (Blackburn, 2018). 


\section{Sampling Procedure}

At each BBD site, American beech trees symptomatic for BBD and non-beech trees with perennial target cankers were identified and sampled. For approximately five trees of each species per site, a maximum of four bark disks harboring fresh perithecia were excised with a 1-cm diameter steel punch and stored in a microtiter plates. Additionally, bark tissue samples ( $1 \mathrm{~mm}$ diameter plugs; $\sim 8$ samples/tree; up to 7 beech trees/site) were taken from symptomatic and asymptomatic trees at BBD and non-BBD sites, respectively. All samples were stored on ice until arriving at the laboratory where samples were stored at $-20^{\circ} \mathrm{C}$ until processing.

\section{Sample Processing}

To process each bark disk, up to five perithecia were removed with a sterile scalpel and cleaned by gently pushing each perithecium through sterile agar to remove debris. Each perithecium was squashed in a $1.5 \mathrm{ml}$ microcentrifuge tube containing $1 \mathrm{ml}$ of sterile $\mathrm{H}_{2} \mathrm{O}$ with a micropestle, vortexed for 15 seconds, and then $300 \mu \mathrm{l}$ of the spore suspension was spread with a cell spreader on glucose-yeast extract agar plates amended with streptomycin sulfate $(10 \mathrm{mg} / 1000 \mathrm{ml})$ and tetracycline hydrochloride (100 $\mathrm{mg} / 1000 \mathrm{ml}$ ) antibiotics (GYE/A). Within 48 hours of plating, five germinating ascospores were subcultured to a new plate, and one isolate was selected for storage at approximately $-20{ }^{\circ} \mathrm{C}$ on glass filter paper. All micro-sampled bark plugs were surface disinfested by soaking for 14 minutes in a 1:10 commercial bleach-water solution then up to four samples were placed onto each GYE/A agar plate. Resulting fungi with morphologies reminiscent of the Nectriaceae were subcultured individually to new plates then stored as previously described. 


\section{Species Identification}

Recovered isolates were grouped and tentatively identified based on colony and macroconidia morphology and a subset of isolates were selected to be confirmed by DNA sequencing. Genomic DNA was extracted from isolates using a Wizard® kit (Promega, Madison, WI, USA) and suspended in $75 \mu \mathrm{l}$ Tris-EDTA (TE) buffer (Amresco, Solon, $\mathrm{OH}, \mathrm{USA})$. PCR reactions were performed for the fungal barcoding genes internal transcribed spacer region (ITS), translation elongation factor 1-alpha (EF-1 $\alpha$ ), $28 \mathrm{~S}$ rDNA (LSU), and $\beta$-tubulin (BTUB). PCR products were generated in $25 \mu \mathrm{l}$ reactions containing $12.5 \mu \mathrm{l}$ Bioline PCR Master Mix (Bioline USA Inc, Taunton, MA), $10.0 \mu \mathrm{l} \mathrm{H} \mathrm{H}_{2} \mathrm{O}, 1.5 \mu \mathrm{l}$ purified DNA, and $1.0 \mu \mathrm{l}$ each of forward and reverse primers (Integrated DNA Technologies, Coralville, IA, USA). All primers, protocols, and their sources are listed in Supplemental Table 2. Positive reactions identified via gel electrophoresis were prepared for sequencing using ExoSAP-IT (Affymetrix, Santa Clara, CA, USA) according to the manufacturer's recommendations and Sanger sequenced using forward PCR primers (Eurofins, Huntsville, AL, USA). Resultant sequences were used to identify species using BLASTn searches, and the best match in the NCBI database was selected for the isolate's identity.

\section{Phylogenetic Analyses}

For all sequences included in the phylogenetic analyses, chromatograms were assessed and clipped using CodonCode Aligner v. 5.1.5. Sequences were then manually corrected for nucleotide misreads by referencing their respective chromatograms. To examine phylogenetic relationships among collected members of Nectriaceae, single-gene and concatenated phylogenetic trees were constructed for all 
Corinectria, Fusarium, and Neonectria species observed in this survey along with additional reference sequences for selected members of Nectriaceae available from NCBI Genbank (Table 3). The other members of the Nectriaceae recovered either lacked sampling depth or adequate reference sequences for the loci of interest to permit meaningful phylogenetic analysis. As such, only BLASTn searches of individual loci were conducted for these species with representative sequences deposited into $\mathrm{NCBI}$ Genbank. For Corinectria, Fusarium, and Neonectria species, each gene was aligned using MAFFT (Katoh and Stanley, 2013) on the Guidance 2.0 server (Landan and Graur, 2008; Sela et al., 2015). Individual residues with Guidance scores $<0.5$ were masked (Macias et al., 2020). A concatenated sequence was generated from single gene alignments using the web tool FaBox (Villesen, 2007).

For single gene and concatenated sequences, maximum-likelihood analyses were completed using MEGA v10.1.7 (Stecher et al., 2020), and Bayesian inference (BI) analyses were completed using MrBayes v. 3.2.7 (Ronquist et al., 2012). For ML analyses, the best-fit nucleotide substitution model was chosen using Model Test AICc scores in MEGA and 1000 bootstrap replicates were used. For BI analyses, MrBayes selected the best fit nucleotide selection model, but the rate of substitution was selected from the Model test AICc scores. The BI runs were stopped once the standard deviation of split frequencies fell below 0.01 . These were then checked for convergence in Tracer v. 1.7.1 (Rambaut et al., 2018). Trees were prepared for publication using FigTree v. 1.4.4 (Rambaut, 2017) and Adobe Illustrator v. 24.1. 


\section{Morphological Measurements}

Over the course of this survey, a species molecularly identified as Nectria magnoliae was recovered from tulip poplar (Liriodendron tulipifera L.) and Fraser magnolia (Magnolia fraseri Walter). Based on a recent study in which isolates from both of these hosts were included, this species was shown to form an independent clade with other members of Neonectria (Chapter 2). A previously published study also supported these relationships (Gräfenhan et al. 2011). Given these phylogenetic relationships, ascospore and conidia measurements were conducted to further characterize this species to permit comparisons with original descriptions provided by Lohman and Watson (1943).

For ascospore measurements, a total of 15 perithecia were processed. Three perithecia were sampled from each of five bark disks representing two geographically separated sites ( $\mathrm{FN}=3$ disks; $\mathrm{SH}=2$ disks). A single perithecium was extracted from the bark disk using a sterile scalpel and squash-mounted on a slide with lactophenol plus cotton blue mountant. Length and width measurements were collected for 25 ascospores per perithecium. All measurements were taken with a Nikon Eclipse E600 compound microscope (Nikon Instruments, Melville, NY, USA) equipped with a Nikon Digital Sight DS-Ri1 microscope camera and Nikon NIS-Elements BR3.2 imaging software.

Conidia measurements were similarly conducted for both micro- and macroconidia. Here, sporodochial masses were harvested from pure four-to six-week-old cultures using a sterile scalpel and mounted onto slides as described above. Both length and width measurements were taken for 50 microconidia were harvested from 
two isolates from each of three geographically separated locations (GK, SH, FN). Samples from SH and FN were collected from L. tulipifera, and samples from GK were collected from $M$. fraseri. Macroconidia were only found associated with one isolate collected from $M$. fraseri at GK and measured as described.

\section{Pathogenicity Assays}

Field inoculations were conducted to further investigate pathogenicity of $N$. magnoliae strains from $L$. tulipifera as well as $N$. faginata and $N$. ditissima strains from beech on birch, striped maple, and beech. For N. magnoliae, a single isolate (NmLt001) recovered from a natural infection on $L$. tulipifera was selected. One isolate of both $N$. ditissima (NdFg002) and $\mathrm{N}$. faginata (NfFg005) recovered from American beech also were included for cross-pathogenicity testing. All study isolates were grown in pure culture on GYE for two-weeks at room temperature. Prior to inoculations, a sterile 1-cm steel punch was used to cut inoculation plugs along the growing edge of the colony. Negative control plugs were cut from sterile GYE plates.

Six each of tulip poplar (L. tulipifera), black birch (Betula lenta), yellow birch (Betula alleghaniensis Britt.), and striped maple (Acer pensylvanicum) trees, located on WVU University Forest were selected for inoculations. Additionally, five American beech trees were selected at this same location. Each tree received an inoculation with $N$. ditissima, N. faginata, and N. magnoliae. In addition to these fungal inoculations, a negative control inoculation with a sterile GYE agar plug also was performed on each study tree.

For each inoculation, a sterile 1-cm leather punch was used to excise bark tissue and create an inoculum reservoir. A colonized or sterile agar plug was then placed into 
the reservoir, and masking tape was applied over the wound to limit inoculum desiccation prior to infection. After six-months, bark tissue was excised from canker margins using a bone-marrow biopsy tool and placed in a 96-well microtiter dish. To ensure accurate canker measurements, a knife was used to remove bark tissue and reveal any underlying necrosis. Length and width measurements were then taken for each canker resulting from inoculation. Recovery of inoculant was achieved as previously described for bark tissue sample processing. All isolate identities were confirmed morphologically.

\section{Statistical analyses}

For pathogenicity measures, a one-way ANOVA was completed to check for differences in canker size and a Tukey-HSD post-hoc test was completed identify significant pairwise differences using the stats $v 3.6 .2$ package within $R \vee 3.6 .3$ statistical software ( $R$ Core Team, 2020). All p-values $<0.05$ were considered significant.

\section{Results}

\section{Survey of nectriaceous fungi}

Perithecia of putative nectriaceous fungi were sampled from 180 trees across 17 sites in WV, MD, VA, NC, and TN (Table 1). Sampling included 12 tree species with black birch, red spruce, and mountain ash being the most abundantly sampled species besides America beech. A total of 1,605 sampled perithecia yielded nine fungal species spanning six genera. The majority of these samples were collected from American beech trees ( $n=1,257$ perithecia). The remaining 348 perithecia were sampled from one of the other 11 host species listed in Table 1. All isolates were grouped initially by 
morphology and a subset was identified with NCBI BLASTn searches using ITS barcoding sequences (Table 2; Supplemental Table 3). For NCBI blast hits, a >98\% sequence identity was used as a cutoff threshold to confirm identification of previously described species.

Of samples collected from American beech trees, approximately $4.2 \%$ (53 out of $1,257)$ of sampled perithecia yielded isolates of $N$. ditissima, while $93.7 \%(1,178$ out of $1,257$ ) yielded $N$. faginata. The remaining $2.1 \%$ (26 out of 1,257$)$ yielded Bionectria ochroleuca. Out of the 13 sites where American beech perithecia were sampled, $N$. ditissima was only recovered from locations FR92 and GK and represented 2.8\% (5 out of 177 ) and $10.8 \%$ (48 out of 443 ) of the viable perithecia collected from those sites, respectively. In one instance, both $N$. ditissima and $N$. faginata were isolated from perithecia occurring on the same bark disk. Bionectria ochroleuca was recovered from 2 of 13 sites, GK and SM.

N. ditissima also was isolated from conspicuous cankers on a number of additional hardwood hosts including yellow birch (Betula allegheniensis), mountain maple (Acer spicatum Lam.) (Figure 2C), black birch (B. lenta) (Figure 2D), and mountain ash (Sorbus americana Marshall) (Figure 2E) (Table 1). Additionally, N. ditissima also was recovered from the main stems of striped maple (A. pensylvanicum) and mountain holly (Ilex mucronata (L.) M.Powell, Savol., \& S.Andrews), though the cankers were inconspicuous and mostly lacking callous ridges (Figure 2A and 2F) (Table 1; Figure 2; Figure 3A). Bionectria ochroleuca was recovered from American beech and American basswood (Tilia americanum L.) (Figure 3G). Three additional members of Nectriaceae also were recovered, albeit less frequently, during this survey 
on tree species found co-occurring with beech in BBD areas or nearby. These included N. magnoliae on tulip poplar (L. tulipifera) and Fraser magnolia (Magnolia fraseri) (Figure 6), Thelonectria veuillotiana ([Roum. \& Sacc.] P. Chaverri \& Salgado) on mountain maple (A. spitacum) and mountain ash (S. americana) (Figure 3F), and Cosmospora obscura (Rossman \& Samuels) on mountain ash (Figure 3H).

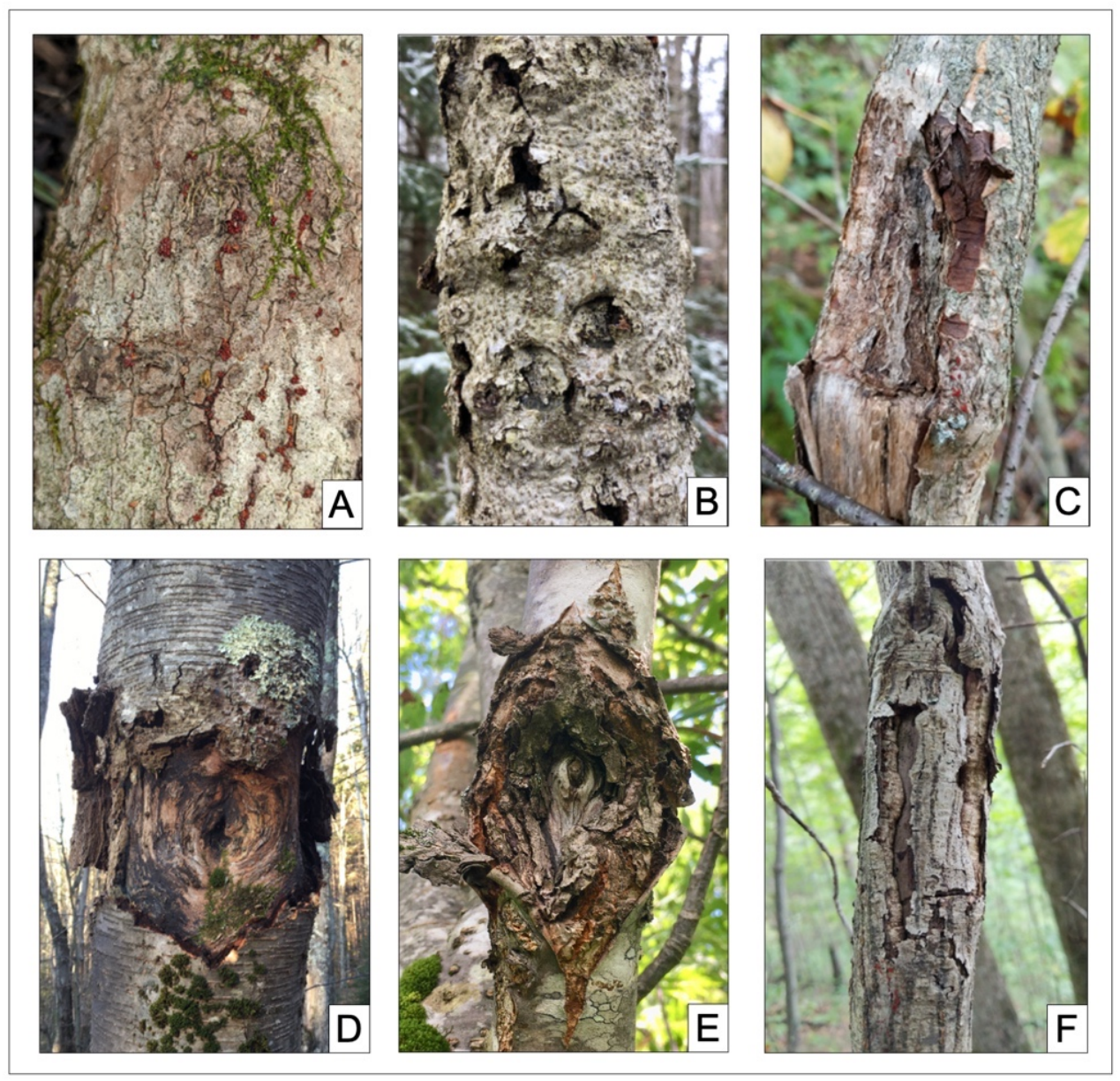

Figure 2: Example Neonectria ditissima canker photos for the following host species: A) mountain holly (Ilex mucronate at SK), B) American beech (Fagus grandifolia at GK), C) mountain maple (Acer spicatum at MM), D) black birch (B. lenta at UF), E) mountain ash (Sorbus Americana at WT), F) striped maple (A. pensylvanicum at SK) 


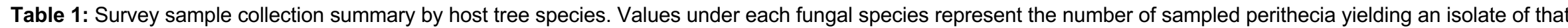
fungus. In total, 1,605 perithecia yielded nine fungal species from ten host tree species at 17 geographically separated sites.

\begin{tabular}{|c|c|c|c|c|c|c|c|c|c|c|c|c|c|}
\hline Host Species & Site & General Location & Trees & Peri & $\begin{array}{c}\text { Neonectr } \\
\text { ia } \\
\text { ditissima }\end{array}$ & $\begin{array}{c}\text { Neonectr } \\
\text { ia } \\
\text { faginata }\end{array}$ & $\begin{array}{c}\text { Neonectr } \\
\text { ia } \\
\text { magnolia } \\
e\end{array}$ & $\begin{array}{c}\text { Neonectr } \\
\text { ia } \\
\text { neomacr } \\
\text { ospora }\end{array}$ & $\begin{array}{c}\text { Bionectri } \\
\text { a } \\
\text { ochroleu } \\
\text { ca }\end{array}$ & $\begin{array}{l}\text { Corinectr } \\
\text { ia sp. }\end{array}$ & $\begin{array}{c}\text { Thyrone } \\
\text { ctria } \\
\text { balsame } \\
\text { a }\end{array}$ & $\begin{array}{c}\text { Cosmos } \\
\text { pora } \\
\text { obscura }\end{array}$ & $\begin{array}{c}\text { Thelonec } \\
\text { tria } \\
\text { veuillotia } \\
\text { na }\end{array}$ \\
\hline \multirow[t]{2}{*}{ Abies balsamea } & MON & $\begin{array}{c}\text { Monongalia County, } \\
\text { WV }\end{array}$ & 1 & 5 & 0 & 0 & 0 & 0 & 0 & 0 & 5 & 0 & 0 \\
\hline & MR & Grayson County, VA & 1 & 5 & 0 & 0 & 0 & 5 & 0 & 0 & 0 & 0 & 0 \\
\hline \multirow{3}{*}{$\begin{array}{c}\text { Acer } \\
\text { pensylvanicum }\end{array}$} & GK & $\begin{array}{c}\text { Randolph County, } \\
\text { WV }\end{array}$ & 3 & 29 & 29 & 0 & 0 & 0 & 0 & 0 & 0 & 0 & 0 \\
\hline & SM & $\begin{array}{c}\text { Cherokee County, } \\
\text { NC }\end{array}$ & 2 & 10 & 10 & 0 & 0 & 0 & 0 & 0 & 0 & 0 & 0 \\
\hline & PSF & Allegany County, MD & 3 & 8 & 8 & 0 & 0 & 0 & 0 & 0 & 0 & 0 & 0 \\
\hline Acer saccharum & GK & $\begin{array}{c}\text { Randolph County, } \\
\text { WV }\end{array}$ & 1 & 5 & 5 & 0 & 0 & 0 & 0 & 0 & 0 & 0 & 0 \\
\hline \multirow{2}{*}{ Acer spicatum } & SM & $\begin{array}{c}\text { Cherokee County, } \\
\text { NC }\end{array}$ & 2 & 8 & 8 & 0 & 0 & 0 & 0 & 0 & 0 & 0 & 0 \\
\hline & MM & Yancey County, WV & 4 & 20 & 9 & 0 & 0 & 0 & 0 & 0 & 0 & 0 & 11 \\
\hline $\begin{array}{c}\text { Betula } \\
\text { alleghaniensis }\end{array}$ & UF & $\begin{array}{c}\text { Monongalia County, } \\
\text { WV }\end{array}$ & 2 & 9 & 9 & 0 & 0 & 0 & 0 & 0 & 0 & 0 & 0 \\
\hline \multirow{4}{*}{ Betula lenta } & $\mathrm{BM}$ & $\begin{array}{c}\text { Randolph County, } \\
\text { WV }\end{array}$ & 1 & 5 & 5 & 0 & 0 & 0 & 0 & 0 & 0 & 0 & 0 \\
\hline & DM & Allegany County, MD & 5 & 12 & 12 & 0 & 0 & 0 & 0 & 0 & 0 & 0 & 0 \\
\hline & $\mathrm{SH}$ & $\begin{array}{c}\text { Monongalia County, } \\
\text { WV }\end{array}$ & 1 & 1 & 1 & 0 & 0 & 0 & 0 & 0 & 0 & 0 & 0 \\
\hline & UF & $\begin{array}{c}\text { Monongalia County, } \\
\text { WV }\end{array}$ & 1 & 5 & 5 & 0 & 0 & 0 & 0 & 0 & 0 & 0 & 0 \\
\hline \multirow{9}{*}{ Fagus grandifolia } & BM & $\begin{array}{c}\text { Randolph County, } \\
\text { WV }\end{array}$ & 7 & 107 & 0 & 107 & 0 & 0 & 0 & 0 & 0 & 0 & 0 \\
\hline & DM & Allegany County, MD & 6 & 80 & 0 & 80 & 0 & 0 & 0 & 0 & 0 & 0 & 0 \\
\hline & $\begin{array}{c}\mathrm{FR} 22 \\
3\end{array}$ & $\begin{array}{c}\text { Randolph County, } \\
\text { WV }\end{array}$ & 5 & 19 & 0 & 19 & 0 & 0 & 0 & 0 & 0 & 0 & 0 \\
\hline & FR92 & $\begin{array}{c}\text { Randolph County, } \\
\text { WV }\end{array}$ & 12 & 177 & 5 & 172 & 0 & 0 & 0 & 0 & 0 & 0 & 0 \\
\hline & GK & $\begin{array}{l}\text { Randolph County, } \\
\text { WV }\end{array}$ & 35 & 467 & 48 & 395 & 0 & 0 & 24 & 0 & 0 & 0 & 0 \\
\hline & SM & $\begin{array}{c}\text { Cherokee County, } \\
\text { NC }\end{array}$ & 16 & 40 & 0 & 38 & 0 & 0 & 2 & 0 & 0 & 0 & 0 \\
\hline & MM & Yancey County, WV & 4 & 10 & 0 & 10 & 0 & 0 & 0 & 0 & 0 & 0 & 0 \\
\hline & MR & Grayson County, VA & 2 & 3 & 0 & 3 & 0 & 0 & 0 & 0 & 0 & 0 & 0 \\
\hline & PSF & Allegany County, MD & 16 & 251 & 0 & 251 & 0 & 0 & 0 & 0 & 0 & 0 & 0 \\
\hline
\end{tabular}




\begin{tabular}{|c|c|c|c|c|c|c|c|c|c|c|c|c|c|}
\hline & $\mathrm{RH}$ & Mitchell County, NC & 5 & 8 & 0 & 8 & 0 & 0 & 0 & 0 & 0 & 0 & 0 \\
\hline & SK & $\begin{array}{c}\text { Pendleton County, W } \\
\text { V }\end{array}$ & 5 & 36 & 0 & 36 & 0 & 0 & 0 & 0 & 0 & 0 & 0 \\
\hline & WT & Grayson County, VA & 6 & 16 & 0 & 16 & 0 & 0 & 0 & 0 & 0 & 0 & 0 \\
\hline & $\begin{array}{l}\text { WVB } \\
\text { G }\end{array}$ & $\begin{array}{c}\text { Monongalia County, } \\
\text { WV }\end{array}$ & 3 & 43 & 0 & 43 & 0 & 0 & 0 & 0 & 0 & 0 & 0 \\
\hline Ilex mucronata & SK & $\begin{array}{c}\text { Pendleton County, W } \\
\text { V }\end{array}$ & 3 & 15 & 15 & 0 & 0 & 0 & 0 & 0 & 0 & 0 & 0 \\
\hline \multirow{2}{*}{$\begin{array}{l}\text { Liriodendron } \\
\text { tulipifera }\end{array}$} & FERN & \multirow{2}{*}{$\begin{array}{c}\text { Tucker County, WV } \\
\text { Monongalia County, } \\
\text { WV }\end{array}$} & 6 & 89 & 0 & 0 & 89 & 0 & 0 & 0 & 0 & 0 & 0 \\
\hline & $\mathrm{SH}$ & & 3 & 15 & 0 & 0 & 15 & 0 & 0 & 0 & 0 & 0 & 0 \\
\hline \multirow{3}{*}{ Picea rubens } & GK & $\begin{array}{c}\text { Randolph County, } \\
\text { WV }\end{array}$ & 4 & 27 & 0 & 0 & 0 & 0 & 0 & 27 & 0 & 0 & 0 \\
\hline & WT & Grayson County, VA & 5 & 38 & 0 & 0 & 0 & 0 & 0 & 38 & 0 & 0 & 0 \\
\hline & MR & Grayson County, VA & 3 & 13 & 0 & 0 & 0 & 0 & 0 & 13 & 0 & 0 & 0 \\
\hline \multirow{3}{*}{$\begin{array}{c}\text { Sorbus } \\
\text { americana }\end{array}$} & MM & Yancey County, WV & 3 & 12 & 7 & 0 & 0 & 0 & 0 & 0 & 0 & 5 & 0 \\
\hline & $\mathrm{RH}$ & Mitchell County, NC & 2 & 7 & 7 & 0 & 0 & 0 & 0 & 0 & 0 & 0 & 0 \\
\hline & WT & Grayson County, VA & 1 & 5 & 5 & 0 & 0 & 0 & 0 & 0 & 0 & 0 & 0 \\
\hline Tilia americana & SM & $\begin{array}{c}\text { Cherokee County, } \\
\text { NC }\end{array}$ & 1 & 5 & 0 & 0 & 0 & 0 & 5 & 0 & 0 & 0 & 0 \\
\hline
\end{tabular}


Table 2: Isolate identification by BLASTn searches using ITS sequences derived from selected representatives of dominant morphologies. Percent identity values represent the lowest identity value among the sequences for each fungal species.

\begin{tabular}{cccc}
\hline Species & Isolate Count & Closest tblastn match & $\%$ identity \\
\hline Neonectria ditissima & $17^{*}$ & Neonectria ditissima & $\geq 98.29 \%$ \\
Neonectria faginata & 5 & Neonectria faginata & $\geq 99.02 \%$ \\
Neonectria magnoliae & 11 & Nectria magnoliae & $\geq 98.38 \%$ \\
Neonectria neomacrospora & 2 & Neonectria & $99.2 \%$ \\
Bionectria ochroleuca & 2 & Bionectria ochroleuca & $\geq 98.5 \%$ \\
Corinectria sp. & 5 & Corinectria fuckeliana & $\geq 94.8 \%$ \\
Thyronectria balsamea & 2 & Thyronectria balsamea & $\geq 99.44 \%$ \\
Cosmospora obscura & 2 & Cosmospora obscura & $\geq 99.04 \%$ \\
Thelonectria veuillotiana & 4 & Thelonectria veuillotiana & $\geq 99.64 \%$ \\
Fusarium babinda & 4 & Fusarium babinda & $\geq 99.8 \%$ \\
\hline
\end{tabular}

*Additional $\mathrm{N}$. ditissima samples sequenced to represent isolates from varied host species

Three members of Nectriaceae also were confirmed on conifer hosts. These included Thyronectria balsamea ([Cooke \& Peck] Seeler) on balsam fir (Abies balsamea (L.) Mill.) and Neonectria neomacrospora ([C. Booth \& Samuels] Mantiri \& Samuels) on Fraser fir (Abies fraseri (Pursh) Poir.) (Figure 3E and 3C, respectively). Additionally, a novel Corinectria sp. on red spruce (Picea rubens Sarg.) was recovered in this survey across high elevation spruce forests in two states (Figure 3D; Figure 4). 


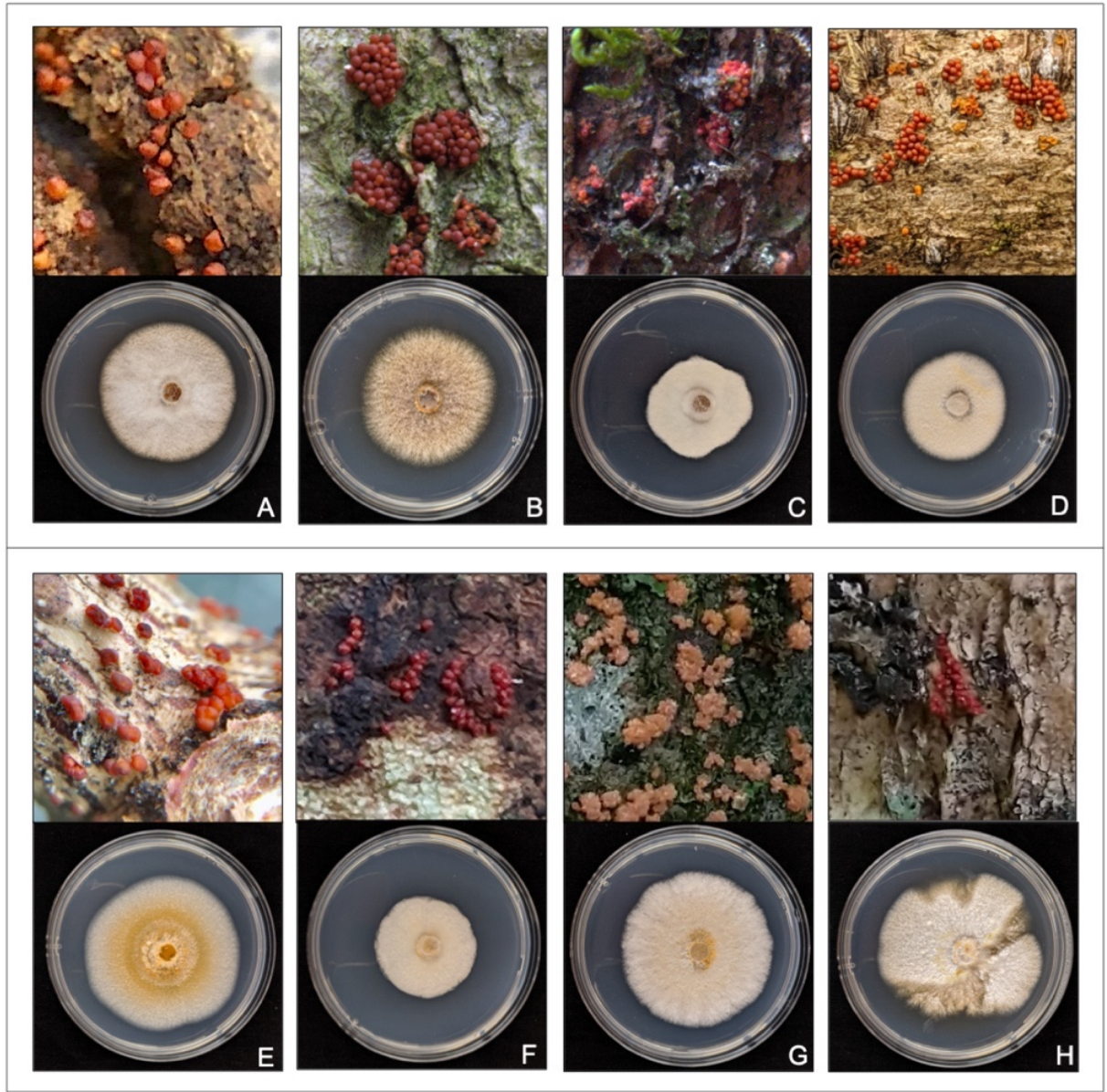

Figure 3: Diversity of Nectriaceae recovered across the central Appalachian Mountains on natural substrate and in culture: A) Neonectria ditissima (shown on striped maple at SK), B) N. faginata (on American beech at BG), C) N. neomacrospora (on Fraser fir at MR), D) Corinectria sp. (on red spruce at GK), E) Thryonectria balsamea (on balsam fir at MON), F) Thelonectria veuillotiana (on mountain maple at MM), G) Bionectria ochroleuca (on American beech at SM), and H) Cosmospora obscura (on mountain ash at $\mathrm{MM})$.

In addition to the previously mentioned nectriaceous fungi recovered from perithecia on symptomatic beech trees and nearby co-occurring trees, a member of the Fusarium babinda species complex (FBSC) was commonly isolated from wood tissues 
of BBD-confirmed American beech trees (31.8\% of bark samples), but it was lacking fruiting bodies (Supplemental Figure 1; Supplemental Table 4). To further investigate this finding, five sites without BBD were identified, and American beech bark tissues were sampled and processed. FBSC was recovered from all confirmed BBD sites but was never recovered from non-BBD sites. Aside from Fusarium babinda, N. faginata and $N$. ditissima were occasionally recovered from beech bark tissues collected but only within BBD sites (Supplemental Table 4).

\section{Phylogenetic Analyses}

Phylogenetic analyses were performed for single genes (ITS, TEF1, TUB, LSU) and for a four-gene concatenated sequence to infer relationships among Neonectria and Corinectria species recovered in the survey. These analyses were supported with the addition of sequence data from several reference strains for both genera (Table 3). Representatives of the Fusarium concolor and Fusarium babinda species complexes were chosen to serve as outgroup taxa that could confirm the identity of Fusarium babinda isolates that were frequently recovered in this study from collected bark tissue samples.

The following results are summarized for relationships with $>70 \%$ bootstrap support within the concatenated phylogeny (Figure 4). Neonectria and Corinectria were resolved as strongly supported (100\%/1.0) sister genera. All included Neonectria species were genealogically exclusive (>83\%/1.0). N. ditissima isolates recovered from diverse host species formed a monophyletic clade with strong support (98\%/1.0). Neonectria neomacrospora was sister to $N$. ditissima $(72 \% / 1.0)$ and isolates recovered in this survey were sister to selected NCBI reference sequences. 
Isolates identified as Nectria magnoliae formed a monophyletic clade within Neonectria $(99 \% / 1.0)$ and was sister to a clade containing $N$. faginata, N. coccinea, $N$. confusa, and $N$. punicea $(92 \% / 1.0)$. The one isolate recovered from Magnolia fraseri (NmMf001) was divergent from all other N. magnoliae isolates recovered from $L$. tulipifera (73\%/0.91). All single-gene trees available in Supplemental Figure 2. 
Table 3: Accession table for sequences used in the phylogenetic analyses of this study. Sequences were either acquired from NCBI or generated as part of this study. Host species and location are also provided where available.

\begin{tabular}{|c|c|c|c|c|c|c|c|}
\hline Species & Strain ID & Host & Locality & BT & LSU & EF1 & ITS \\
\hline Fusarium babinda & FbFg001 & $\begin{array}{l}\text { Fagus } \\
\text { grandifolia }\end{array}$ & $\begin{array}{c}\text { Allegany County, MD, } \\
\text { USA }\end{array}$ & TBD & TBD & TBD & TBD \\
\hline F. babinda & FbFg002 & F. grandifolia & $\begin{array}{c}\text { Randolph County, WV, } \\
\text { USA }\end{array}$ & TBD & TBD & TBD & TBD \\
\hline F. babinda & FbFg003 & F. grandifolia & $\begin{array}{c}\text { Allegany County, MD, } \\
\text { USA }\end{array}$ & TBD & TBD & TBD & TBD \\
\hline F. babinda & FbFg004 & F. grandifolia & $\begin{array}{c}\text { Monongalia County, } \\
\text { WV, USA }\end{array}$ & TBD & TBD & TBD & TBD \\
\hline F. concolor & NRRL 13994 & Hordeum sp. & Uruguay & U61549.1 & U61653.1 & --- & U61679.1 \\
\hline F. concolor & ВССМ9748 & Homo sapiens & Spain & KJ125965.1 & KJ126557.1 & --- & KJ125669.1 \\
\hline $\begin{array}{l}\text { Neonectria } \\
\text { coccinea }\end{array}$ & AF3707 & F. sylvatica & Slovakia & TBD & TBD & --- & TBD \\
\hline N. coccinea & AR3691 & F. sylvatica & Romania & TBD & TBD & --- & TBD \\
\hline N. coccinea & CBS 118916 & F. sylvatica & Romania & DQ789840.1 & KC660601.1 & KC660442.1 & KC660505.1 \\
\hline N. coccinea & CBS 119158 & Fagus sp. & Germany & KC660727.1 & KC660620.1 & JF268734.1 & KC660521.1 \\
\hline N. confusa & JL2009a-5741 & --- & --- & JF268721.1 & --- & JF268736.1 & JF268760.1 \\
\hline N. confusa & CBS 127484 & --- & China & KM515886.1 & KM515933.1 & --- & KM515889.1 \\
\hline N. ditissima & $\mathrm{NdBa001}$ & $\begin{array}{c}\text { Betula } \\
\text { alleghaniensis }\end{array}$ & $\begin{array}{c}\text { Monongalia County, } \\
\text { WV, USA }\end{array}$ & TBD & TBD & TBD & TBD \\
\hline N. ditissima & NdSam001 & $\begin{array}{l}\text { Sorbus } \\
\text { americana }\end{array}$ & $\begin{array}{c}\text { Grayson County, VA, } \\
\text { USA }\end{array}$ & TBD & TBD & TBD & TBD \\
\hline
\end{tabular}




\begin{tabular}{|c|c|c|c|c|c|c|c|}
\hline N. ditissima & NdAp002 & $\begin{array}{c}\text { Acer } \\
\text { pensylvanicum }\end{array}$ & $\begin{array}{c}\text { Swain County, NC, } \\
\text { USA }\end{array}$ & TBD & TBD & TBD & TBD \\
\hline N. ditissima & $\mathrm{NdAs002}$ & Acer spicatum & $\begin{array}{c}\text { Yancey County, WV, } \\
\text { USA }\end{array}$ & TBD & TBD & TBD & TBD \\
\hline N. ditissima & Ndll001 & Ilex mucronata & $\begin{array}{c}\text { Pendleton County, WV, } \\
\text { USA }\end{array}$ & TBD & TBD & TBD & TBD \\
\hline N. ditissima & NdBI002 & Betula lenta & $\begin{array}{c}\text { Allegany County, MD, } \\
\text { USA }\end{array}$ & TBD & TBD & TBD & TBD \\
\hline N. ditissima & NdAsa001 & Acer saccharum & $\begin{array}{l}\text { Randolph County, WV, } \\
\text { USA }\end{array}$ & TBD & TBD & TBD & TBD \\
\hline N. ditissima & $\mathrm{NdFg002}$ & F. grandifolia & $\begin{array}{c}\text { Randolph County, WV, } \\
\text { USA }\end{array}$ & TBD & --- & TBD & TBD \\
\hline N. ditissima & NdFg006 & F. grandifolia & $\begin{array}{c}\text { Randolph County, WV } \\
\text { USA }\end{array}$ & TBD & TBD & TBD & TBD \\
\hline N. ditissima & NdFg001 & F. grandifolia & $\begin{array}{l}\text { Randolph County, WV } \\
\text { USA }\end{array}$ & TBD & TBD & TBD & TBD \\
\hline N. ditissima & CBS 100.316 & --- & Ireland & HM352864.1 & HM364311.1 & HM364350.1 & HM364298. \\
\hline N. ditissima & CBS 226.31 & F. sylvatica & Tharandt, Germany & DQ789869.1 & AY677330.1 & JF735783.1 & JF735309.1 \\
\hline N. faginata & CBS 118938 & F. grandifolia & PA, USA & DQ789846.1 & KC660614.1 & KC660446.1 & KC660508.1 \\
\hline N. faginata & CBS 217.67 & F. grandifolia & $\begin{array}{l}\text { New Brunswick, } \\
\text { Canada }\end{array}$ & JF268730.1 & HQ840382.1 & JF268746.1 & HQ840385.1 \\
\hline N. faginata & NfFg014 & F. grandifolia & $\begin{array}{c}\text { Allegany County, MD, } \\
\text { USA }\end{array}$ & TBD & TBD & TBD & TBD \\
\hline N. faginata & NfFg007 & F. grandifolia & $\begin{array}{c}\text { Swain County, NC, } \\
\text { USA }\end{array}$ & TBD & TBD & TBD & TBD \\
\hline N. faginata & NfFg005 & F. grandifolia & $\begin{array}{c}\text { Randolph County, WV, } \\
\text { USA }\end{array}$ & TBD & TBD & --- & TBD \\
\hline
\end{tabular}




\begin{tabular}{|c|c|c|c|c|c|c|c|}
\hline N. faginata & NfFg015 & F. grandifolia & $\begin{array}{c}\text { Monongalia County, } \\
\text { WV, USA }\end{array}$ & TBD & TBD & --- & TBD \\
\hline N. magnoliae & NmLt001 & $\begin{array}{l}\text { Liriodendron } \\
\text { tulipifera }\end{array}$ & $\begin{array}{c}\text { Tucker County, WV, } \\
\text { USA }\end{array}$ & TBD & TBD & TBD & TBD \\
\hline N. magnoliae & NmLt002 & L. tulipifera & $\begin{array}{c}\text { Tucker County, WV, } \\
\text { USA }\end{array}$ & TBD & --- & TBD & TBD \\
\hline N. magnoliae & NmLt003 & L. tulipifera & $\begin{array}{c}\text { Monongalia County, } \\
\text { WV, USA }\end{array}$ & TBD & TBD & TBD & TBD \\
\hline N. magnoliae & NmLt004 & L. tulipifera & $\begin{array}{c}\text { Monongalia County, } \\
\text { WV, USA }\end{array}$ & TBD & -- & TBD & TBD \\
\hline N. magnoliae & NmMf001 & Magnolia fraseri & $\begin{array}{c}\text { Randolph County, WV, } \\
\text { USA }\end{array}$ & TBD & TBD & TBD & TBD \\
\hline N. punicea & CBS 119532 & F. sylvatica & Slovakia & KC660728.1 & KC660563.1 & KC660439.1 & KC660503.1 \\
\hline N. punicea & CBS 242.29 & Rhamus sp. & Germany & DQ789873.1 & MH866520.1 & DQ789730.1 & MH855054.1 \\
\hline N. neomacrospora & $\mathrm{NnAb002}$ & Abies fraseri & $\begin{array}{c}\text { Grayson County, VA, } \\
\text { USA }\end{array}$ & TBD & TBD & TBD & TBD \\
\hline N. neomacrospora & NnAb001 & A. fraseri & $\begin{array}{c}\text { Grayson County, VA, } \\
\text { USA }\end{array}$ & TBD & TBD & TBD & TBD \\
\hline N. neomacrospora & CBS 118985 & $\begin{array}{c}\text { Tsuga } \\
\text { heterophylla }\end{array}$ & Canada & DQ789890.1 & HQ840380.1 & JF268755.1 & HQ840389.1 \\
\hline N. neomacrospora & CBS 118984 & Abies balsamea & Canada & DQ789882.1 & HQ840379.1 & JF735787.1 & HQ840388.1 \\
\hline N. hederae & IMI 058770a & Hedera helix & United Kingdom & DQ789895.1 & KC660617.1 & KC660461.1 & -- \\
\hline N. hederae & CBS 714.97 & H. helix & Netherlands & DQ789878.1 & KC660616.1 & KC660461.1 & QGQB00000000 \\
\hline N. ramulariae & CBS 151.29 & Malus sylvestris & United Kingdom & DQ789863.1 & AY677333.1 & HM054091.1 & AY677291.1 \\
\hline N. ramulariae & CBS 182.36 & M. sylvestris & --- & JF735439.1 & MH867276.1 & JF735792.1 & MH855762.1 \\
\hline
\end{tabular}




\begin{tabular}{|c|c|c|c|c|c|c|c|}
\hline Corinectria sp. & CspPr004 & Picea rubens & $\begin{array}{c}\text { Randolph County, WV } \\
\text { USA }\end{array}$ & TBD & TBD & TBD & TBD \\
\hline Corinectria sp. & CspPr007 & P. rubens & $\begin{array}{c}\text { Randolph County, WV, } \\
\text { USA }\end{array}$ & TBD & TBD & TBD & TBD \\
\hline Corinectria sp. & CspPr002 & P. rubens & $\begin{array}{c}\text { Grayson County, VA, } \\
\text { USA }\end{array}$ & TBD & TBD & TBD & TBD \\
\hline Corinectria sp. & CspPr008 & P. rubens & $\begin{array}{c}\text { Grayson County, VA, } \\
\text { USA }\end{array}$ & --- & TBD & TBD & TBD \\
\hline C. tsugae & CBS 788.69 & T. heterophylla & Canada & KM232020.1 & MH871201.1 & --- & KM231763.1 \\
\hline C. fuckeliana & IMI 342668 & Picea sp. & Switzerland & KJ022340.1 & KJ022070.1 & KJ022404.1 & KJ022021.1 \\
\hline C. fuckeliana & CBS 239.29 & Picea sitchensis & Scotland & DQ789871.1 & HQ840377.1 & JF268748.1 & HQ840386.1 \\
\hline C. constricta & LASBE 266 & Pinus radiata & Chile & KY636417.1 & --- & KY636410.1 & --- \\
\hline
\end{tabular}




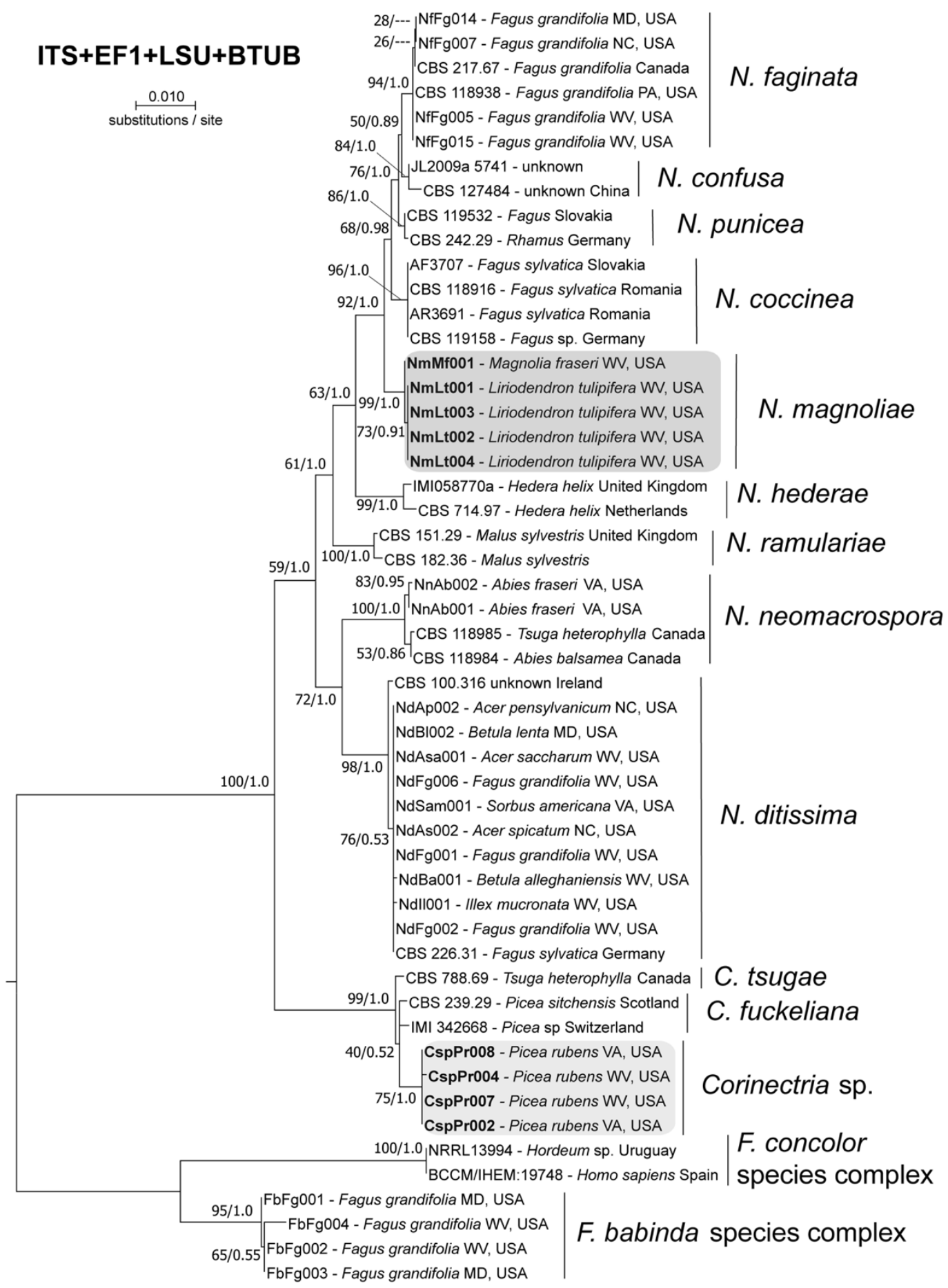

Figure 4: Four-gene (ITS, BTUB, ACTIN, EF1) concatenated phylogeny tree of

Neonectria spp., Corinectria spp. and outgroups. Topology and branch lengths are from

the $\mathrm{ML}$ analysis. For each node supported in the ML analysis, bootstrap support and

posterior probabilities are indicated (ML/BI). 


\section{Pathogenicity Assay}

A field pathogenicity assay was conducted to further characterize $N$. magnoliae and test the pathogenicity of $N$. faginata and $N$. ditissima isolates originating from American beech on alternative host species including those not previously included in cross pathogenicity assays. Overall, $N$. ditissima produced significantly larger cankers $(p<0.05)$ than all other treatments on striped maple, yellow birch, and black birch (Figure 5A-C, respectively). N. faginata and N. magnoliae failed to produce cankers significantly larger than the negative control on any of the aforementioned hosts. On American beech, $N$. ditissima produced larger cankers than $N$. magnoliae and the negative control, but $N$. ditissima and $N$. faginata canker sizes were not significantly different $(p=0.71)($ Figure 5D). N. faginata produced a larger canker than N. magnoliae, although not statistically significant, and negative control on American beech $(p=0.19$ and 0.23 , respectively). N. magnoliae produced a significantly larger canker on tulip poplar than all other treatments $(p<0.0001)$ (Figure $5 \mathrm{E})$. The treatment isolate was recovered from all respective inoculations. 


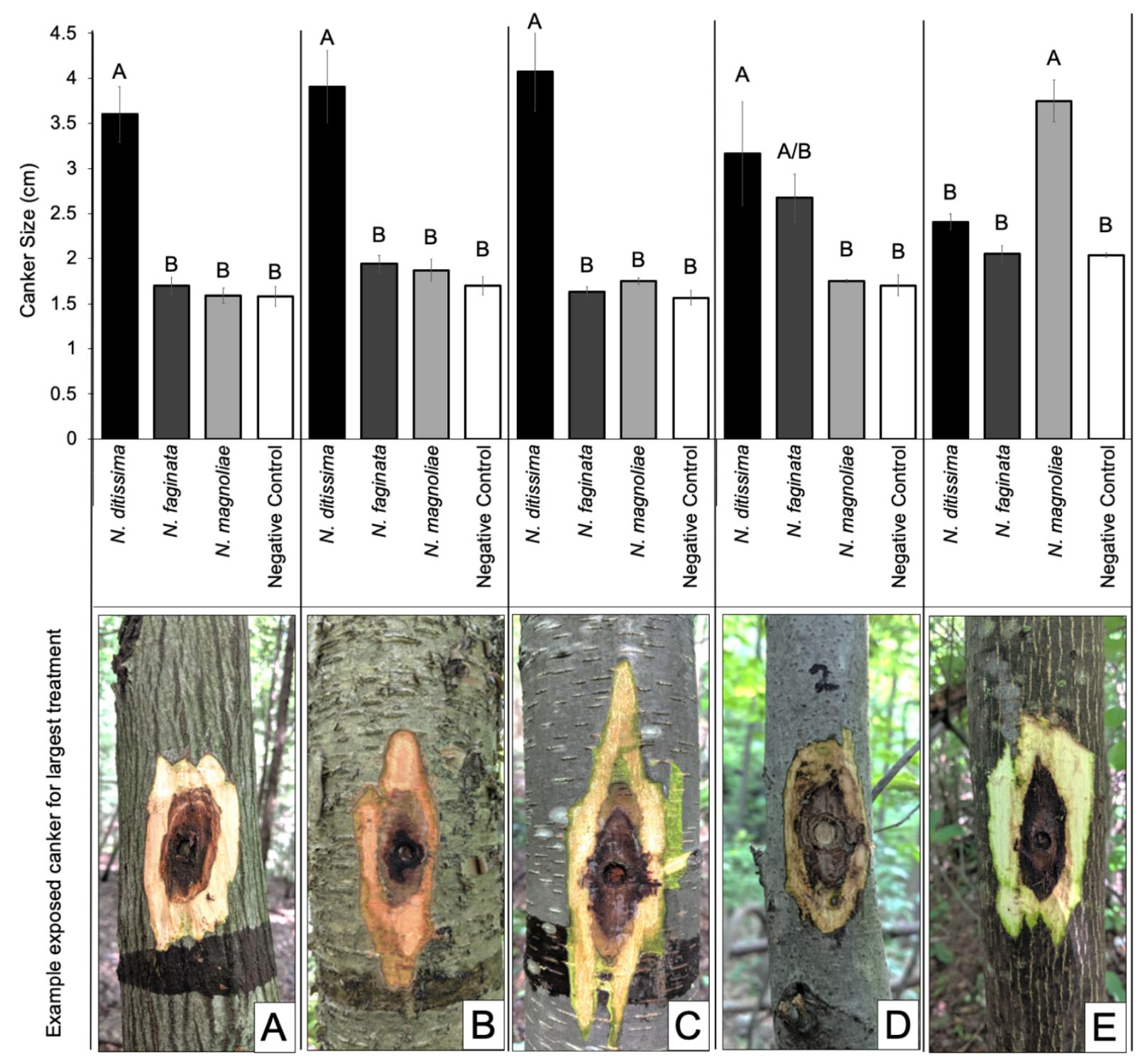

Figure 5: Pathogenicity results and representative photos for each host species tested: A) striped maple (Acer pensylvanicum), B) yellow birch (Betula alleghaniensis), C) black birch (B. lenta), D) American beech (Fagus grandifolia), and E) tulip poplar (Liriodendron tulipifera). Letters designate significant differences at $p<0.05$. All sample sizes are 6 trees, except for American beech $(n=5)$. 


\section{Morphological Characterization of Neonectria magnoliae}

The morphological features of Neonectria magnoliae are summarized here and illustrated in Figure 6. Cankers produced by N. magnoliae on tulip poplar (Figure 6A) were reminiscent of the perennial target cankers produced by $N$. ditissima, but these cankers often appeared irregular and less descript (e.g. lacking concentric rings of callous tissue), especially for infections on M. fraseri (Figure 6B-C). Perithecia occur singly or in aggregates on bark tissue or directly on exposed wood surrounding the outer canker margins and typically emerge from thin stroma tissues as reddish-brown globous body with a distinct ostiole then fade to brown as they age (Figure 6D). Asci appear truncated and bear eight ascospores (Figure 6E). Ascospores ((11.3-) $13.1-$ $15.3(-17.7) \mu \mathrm{m} \times(4.0-) 5.6-7.4(-9.1) \mu \mathrm{m})$ are uniseptate with rounded ends, constricted at the septum, hyaline, and warty on the surface (Figure 6F-G). Neonectria magnoliae cultures have a white surface with an orange-red subsurface after $10 \mathrm{~d}$ on PDA (Figure 6H). Microconidial sporodochia and condiophores are regularly produced in culture (Figure 6I). Sporodochia are slimy masses with a cream to buff color. Conidiophores appear white and are penicillately branched. Microconidia ((5.7-) 8.6 $11.0(-13.7) \mu \mathrm{m} \times(3.0-) 4.0-5.3(-6.4) \mu \mathrm{m})$ are hyaline, straight, and aseptate with rounded ends (Figure $6 \mathrm{~K}$ ). Microconidia are abundantly produced in culture on aerial conidiophores and within sporodochia. Macroconidia ((42.1-) $47.5-61.7$ (-67.3) $\mu \mathrm{m} \mathrm{x}$ (5.5-) $6.8-8.4(-9.4) \mu \mathrm{m})$ are primarily $4-5$ septate, cylindrical with rounded ends, slightly curved, and hyaline (Figure 6J). Macroconidia, while they do occur, are found less frequently in sporodochia. 

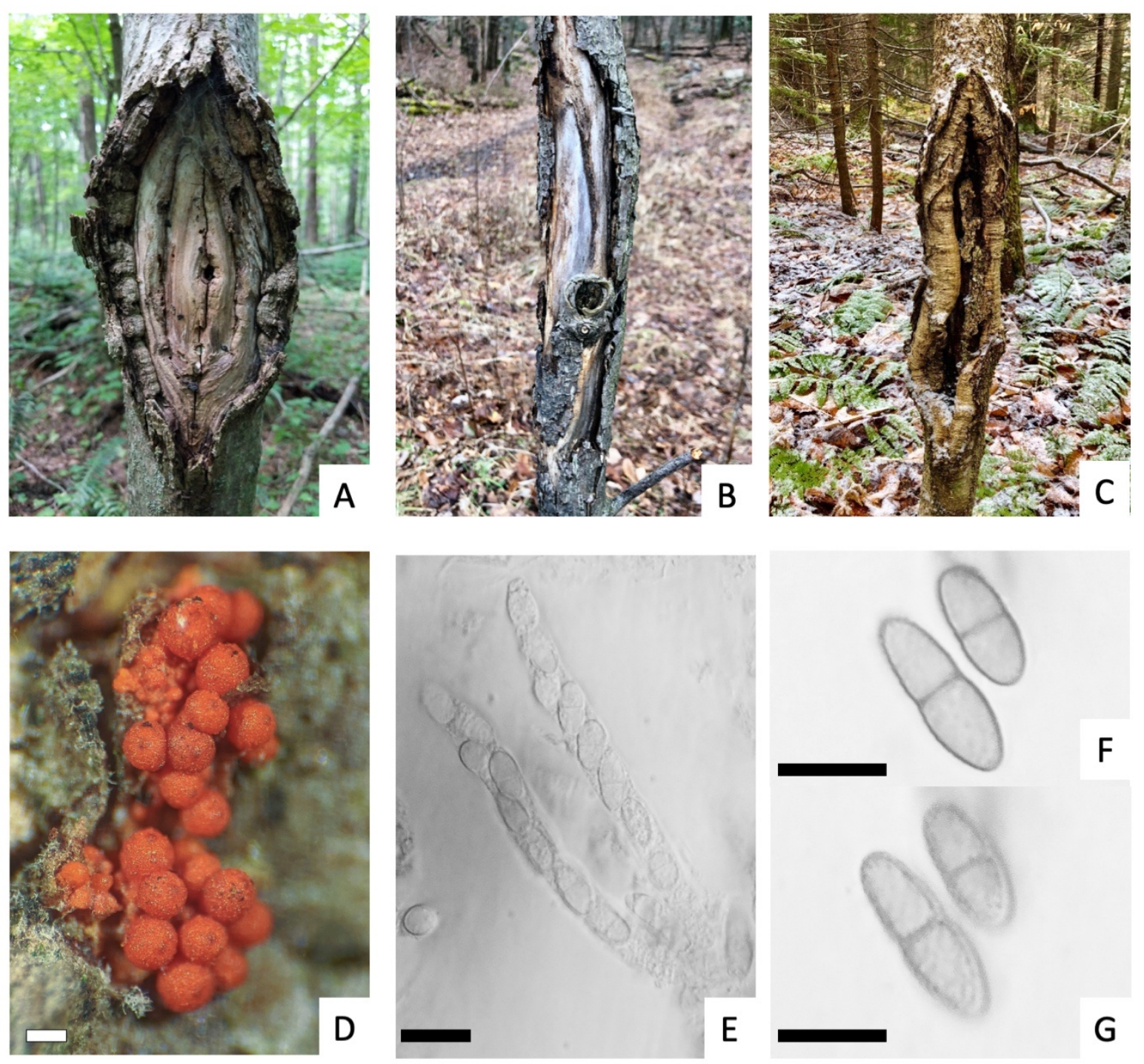

F
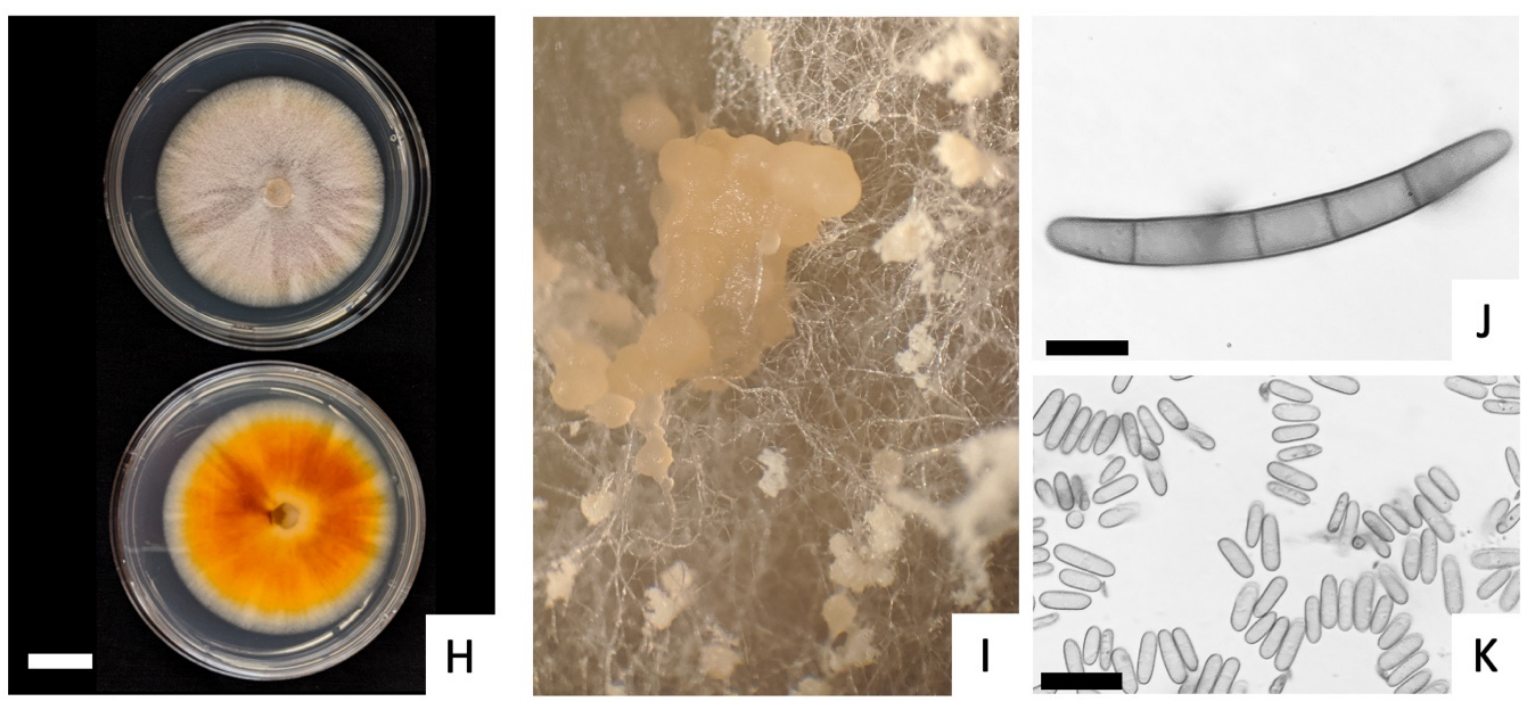
Figure 6: Neonectria magnoliae: A) Example canker on Liriodendron tulipifera with perennial target canker morphology; B) Example canker on L. tulipifera exhibiting less distinct canker morphology; C) Example canker on Magnolia fraseri; D) N. magnoliae perithecia erupting from L. tulipfera bark tissues; E) Asci containing eight ascospores each; F) Ascospores; G) Ornamented surface of ascospores; H) Top and bottom of 7day old culture photos on PDA; I) Creamy microconidial sporodochia and aerial conidiophores; J) Macroconidia with four septations; K) Microconidia of varying lengths. Scale bars $=250 \mu \mathrm{m}$ for panel $\mathrm{D} ; 20 \mu \mathrm{m}$ for panels $\mathrm{E}, \mathrm{K} ; 10 \mu \mathrm{m}$ for panels $\mathrm{F}, \mathrm{G}, \mathrm{J} ; 20$ $\mathrm{mm}$ for panel $\mathrm{H}$.

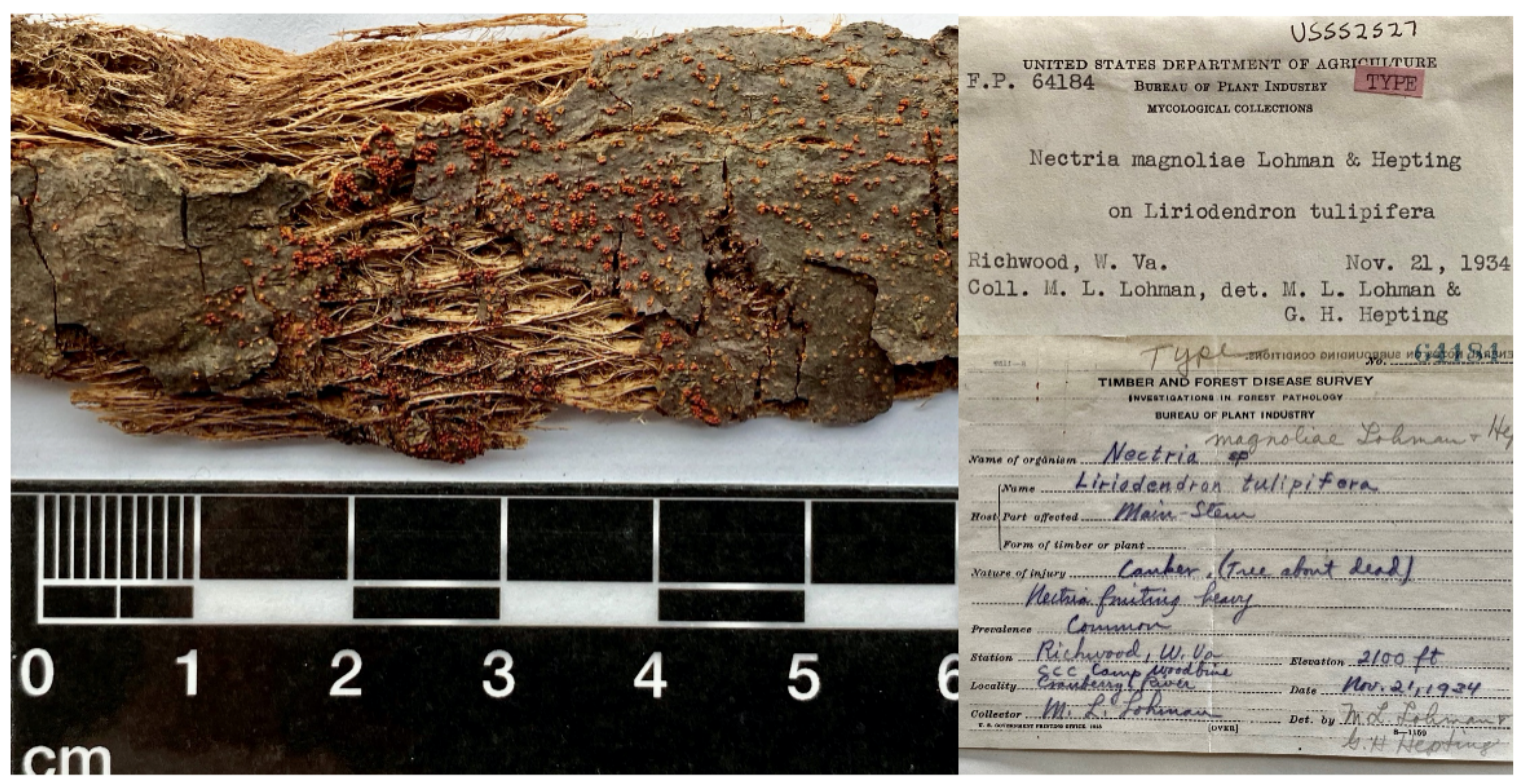

Figure 7: Neonectria magnoliae holotype (BPI 552527 [No. 64184]) submitted by Lohman and Heptig in 1934. Sample consists of $N$. magnoliae fruiting on bark of tulip poplar (Liriodendron tulipifera) collected in Richwood, West Virginia, USA. Photos provided by Lisa A Castlebury, Ph.D, Acting Research Leader and Collections Director, USDA ARS MNGDBL. 


\section{Taxonomy}

Neonectria magnoliae (Lohman \& Hepting) C.M. Stauder \& M.T. Kasson comb. nov.

E Nectria magnoliae M.L. Lohman \& Hepting, Lloydia 6: 91. 1943.

Similar to Neonectria coccinea; ascospores $13.1-15.3 \times 5.6-7.4 \mu \mathrm{m}$ with regularly scattered warts; Microconidia aseptate; Macroconidia rare except for isolates from Magnolia fraseri. When present slightly curved $4-5$ septate.

Holotype: UNITED STATES. West Virginia: Richwood. Civilian Conservation Corps Camp Woodbine (currently Camp Richwood) along the Cranberry River, on bark of almost dead Liriodendron tulipifera, Nov. 21, 1934, M. L. Lohman, BPI 552527 (No. 64184) (Figure 7).

Specimens examined: UNITED STATES. North Carolina: Asheville. Bent Creek Experimental Forest, on cankered Liriodendron tulipifera, October 12, 1934, M. L. Lohman, BPI 552527 (No. 64187; CBS 380.50). MB\#288753. ITS and RPB2 sequences available in NCBI Genbank, accessions $\underline{\mathrm{MH} 856671}$ and $\underline{\mathrm{HQ897713}}$, respectively. West Virginia: Monongalia County. Snake Hill Wildlife Management Area, on cankered $L$. tulipifera, October 1, 2018, C. M. Stauder and M. T. Kasson, NmLt003, NmLt004, ITS sequences available in NCBI Genbank, accessions $\underline{\text { TBD }}$, $\underline{\text { TBD }}$, respectively. Tucker County. Fernow Experimental Forest, on cankered L. tulipifera, July 12, 2018, C.M. Stauder, A. M. Macias, M. T. Kasson, NmLt001, NmLt002, ITS sequences available in NCBI Genbank, accessions $\underline{\text { TBD, }} \underline{\text { TBD, }}$, respectively. Randolph County. Gaudineers Knob, on cankered Magnolia fraseri, M.T. Kasson, NmMf001, ITS sequences available in NCBI Genbank, accessions $\underline{\text { TBD. }}$ 
Habitat and distribution: on bark and exposed wood around cankers on living and dead Liriodendron tulipifera L. in Connecticut, Ohio, West Virginia, Virginia, Tennessee and North Carolina, Magnolia fraseri Walt. trees in West Virginia and Tennessee, and M. tripetala L. in West Virginia. Trees include both overtopped and overstory trees. Often found in forested stands co-occurring with other hosts harboring conspicuous $N$. ditissima target cankers.

Descriptions and illustrations: See Lohman and Watson (1943).

\section{Discussion}

In this study, we sampled and characterized the diversity of Nectriaceae across the central Appalachian Mountains. Over the course of the survey, ten species of Nectriaceae belonging to Bionectria, Corinectria, Cosmospora, Fusarium, Neonectria, Thelonectria, and Thyronectria were recovered from twelve tree hosts spanning 17 sites across six states (Table 1; Figure 3). The limited recovery of Cosmospora obscura, Neonectria neomacrospora, Thelonectria balsamea, and Thyronectria veuillotiana limits the ability to explore their ecology, but follow-up surveys/studies on these species could provide further insight into lifestyles of these fungi.

Other recovered members of Nectriaceae were either widespread or found in sufficient numbers at fewer locations, thereby providing adequate resolution and/or insight into their ecology. These species included the following: Neonectria magnoliae from symptomatic tulip poplar and Fraser magnolia; $N$. ditissima from eight hosts; $N$. faginata, Bionectria ochroleuca, and Fusarium babinda from American beech; and a novel Corinectria sp. from red spruce (Table 1; Figure 3). 
Neonectria magnoliae was first described as Nectria magnoliae by Lohman and Watson (1943) as having some overlapping characteristics of both Neonectria ditissima (formerly Nectria galligena) and Neonectria coccinea (formerly Nectria coccinea) but also with notable morphological features including progressive changes in coloration of perithecia and notable spore size differences. Many former members of the genus Nectria have since been reclassified into different genera (Brayford et al., 2004; Castlebury et al., 2006; Mantiri et al., 2001; Rossman et al., 1999). However, none of these studies considered Nectria magnoliae, and therefore, its taxonomy was never properly resolved with one erroneous exception: Castlebury and colleagues (2006) included one isolate of Nectria magnoliae from tulip poplar from Tennessee in a phylogenetic study of Neonectria. These authors concluded that N. magnoliae was a synonym of $N$. ditissima, based on the data available at the time. However, based on BLASTn searches against NCBI Genbank and pairwise blasting of $N$. magnoliae isolates with $N$. ditissima strain CBS 118919, it's clear that both $N$. magnoliae and $N$. ditissima infect tulip poplar and strain CBS 118919, sequenced by Castebury et al. (2006) was in fact N. ditissima. NCBI BLASTn searches with ITS and RPB2 sequences generated for field-collected $N$. magnoliae isolates had $98.4 \%-100 \%$ sequence similarity with $77-100 \%$ sequence coverage with a paratype of Nectria magnoliae (CBS 380.50 Genbank Accessions NR_160076 and HQ897713, respectively) (Supplemental Table 3). Additionally, the four-gene concatenated phylogeny placed $N$. magnoliae isolates as a monophyletic ingroup within Neonectria (Figure 4).

Ascospore measurements and general morphological descriptions provided in the type description for N. magnoliae (Lohman and Watson, 1943) were comparable 
with those observed in this study (Figure 6). Notable was the infrequency of macroconidia from fresh cultures on general growth media. Interestingly, this trend did not hold for all isolates as several of the $N$. magnoliae from Fraser magnolia produced abundant macroconidia even in week-old cultures. The only isolate of N. magnoliae from magnolia also showed some sequence divergence from all tulip poplar isolates (Figure 4). Further characterization of magnolia and tulip-polar isolates are needed to confirm whether these notable differences are biologically significant. The included pathogenicity trial confirmed the pathogenicity of $N$. magnoliae on tulip poplar but not on other hosts tested (Figure 5). Interestingly, $N$. ditissima did not produce cankers significantly different from the negative control on tulip-poplar despite the previous work by Castlebury et al. (2006) confirming tulip-poplar as a host of $N$. ditissima (Figure 5). Earlier work by Lohman and Watson (1934) also detail cross-pathogenicity experiments in North Carolina where diverse $N$. ditissima strains from various hosts caused significantly larger cankers on tulip poplar compared to N. magnoliae. Follow-up studies are needed for cross pathogenicity tests of $\mathrm{N}$. magnoliae from different hosts as well as N. ditissima isolates from tulip poplar. Together, these results provide evidence to support the transfer of Nectria magnoliae into the genus Neonectria.

Neonectria ditissima and $N$. faginata represented a majority of isolates recovered during this study (Table 1). Although this study did not uncover cryptic native plant reservoirs of $N$. faginata, $N$. ditissima was confirmed from eight plant hosts including three of which that may represent the first reports: Acer spicatum, llex mucronata, and Sorbus americana (Table 1). Interestingly, not all hosts had characteristic perennial target cankers (Figure 2). Instead, Acer pensylvanicum and Ilex mucronata exhibited 
vascular cambium necrosis and perithecia production without an apparent outward host response (e.g. callous ridges, bark malformation). Perithecia production also varied across hosts with black birch cankers consistently yielded some perithecia and mountain ash cankers rarely yielding perithecia.

Cross-pathogenicity assays among $N$. ditissima strains demonstrated the lack of host specificity between isolates recovered from American beech or alternate host species (Figure 5). Additionally, a recent study confirmed the lack of mating barriers among N. ditissima strains recovered from varying host tree species (Chapter 2). Together, these results provide additional observational evidence describing the extent of the generalist nature of $N$. ditissima as a ubiquitous phytopathogen in eastern North America capable of infecting many hardwood species.

Neonectria faginata was the most recovered member of the Nectriaceae in this study, though it was exclusive to American beech (Table 1). The identification of a cryptic reservoir may have provided additional evidence supporting the geographic origin of $N$. faginata as eastern North America. Alternatively, N. faginata may be an exotic species simply capable of infecting as-yet-unidentified host species given this remains a possibility for American beech. Either way, no evidence of an alternate host for $N$. faginata was uncovered in this survey. Follow-up studies should focus on cankers lacking perithecia and/or asymptomatic host tissue similar to what has been previously described for epiphytic N. coccinea on European beech (Chapela and Boddy, 1988; Hendry et al., 2002).

Previous assertions of the eventual dominance of $N$. faginata in the BBD pathosystem (Houston, 1994; Kasson and Livingston, 2009) were confirmed across the 
study sites. In total, $N$. faginata was recovered from $93.7 \%$ of BBD samples collected in this survey (from 13 of 13 BBD sites) while $N$. ditissima was only recovered from $4.2 \%$ of BBD samples (from two of the 13 BBD sites) (Table 1).

Nevertheless, $N$. ditissima still appears to play a minor role in BBD in its more advanced stages. The pathogenicity trial demonstrated the potentially increased pathogenicity of $N$. ditissima on American beech when compared to $N$. faginata (Figure 5). While the interaction of the scale insect may significantly alter the host's physiology and thus, defense responses to fungal infections, these results appear to indicate that an increased virulence of $N$. faginata may not be responsible for its dominance in the BBD pathosystem.

One additional possibility may be related to its production of perithecia and the resulting inoculum potential. For example, taxonomic descriptions of $N$. ditissima and $N$. faginata illustrate differences in their production of perithecia. In general, N. ditissima is described as bearing fewer, scattered perithecia while $N$. faginata can be found to produce larger aggregates in higher densities (Castlebury et al., 2006; Lohman and Watson, 1943). Sampling of $N$. ditissima perithecia across eight hosts generally supports these previous observations (Table 1). Seasonal differences in fruiting could explain differential abundances in these two fungi, but $N$. ditissima isolates were recovered from beech and non-beech hosts during the same sampling periods in which sampling of BBD trees resulted in high abundances of $N$. faginata. Therefore, seasonality of fruiting does not appear to have been an apparent factor in this study. Further, several studies report ascospores as the predominant spore type in the environment and more specifically, the dominant infective spore type (Crane et al., 
2009; Lortie and Kuntz, 1963). While these are general descriptive observations and not quantitative comparisons, differences in fruiting habits may explain the eventual abundance of $N$. faginata due to a significantly increased inoculum potential.

Bionectria ochroleuca also was recovered from BBD impacted beech trees at two total sites in West Virginia and North Carolina, representing 2.1\% of all perithecia sampled from beech (Table 1; Figure 3). This fungus previously had been implicated in BBD although its geographic range was not well studied (Houston et al., 1987). Here we update the known distribution of this fungus to include West Virginia and North Carolina. Observations of this fungus on successfully colonized trees supports previous studies, which indicate this fungus is mycoparasitic (Barnett and Lilly, 1962; Jager et al. 1979; Turhan, 1993). Isolates of this fungus also were recovered from American basswood in North Carolina during these surveys (Table 1). This appears to be the first report of this fungus on American basswood in the U.S.

An additional fungus recovered consistently from beech with BBD was a member of the Fusarium babinda species complex (FBSC), although it did not produce perithecia, like all other Nectriaceae recovered in this study. Instead, members of the FBSC were often found colonizing the base of Neonectria perithecia or from necrotic tissue around Neonectria fruiting bodies. After its discovery in single spore plates from Neonectria perithecia, follow-up studies using micro-sampled bark tissue revealed this fungus was present in $31.8 \%$ of BBD-positive bark samples at $100 \%$ of BBD sampled sites but not present in any beech bark samples taken from healthy asymptomatic trees outside confirmed BBD epicenters (Supplemental Table 4). Its prevalence from BBD impacted stands, but lack of previous reporting, indicates either a localized (albeit 
unclear) role or novel component in BBD pathosystem. However, a re-assessment of raw data and culture photos taken by the senior author during $2005-2006$ as part of his BBD M.S. research in Maine (Kasson and Livingston, 2009) uncovered a Fusarium with identical morphology to $F$. babinda in three of three sites sampled for which culture data was collected (Supplemental Figure 1; See Kasson and Livingston, 2007, Figure 17A). This clearly indicates a more widespread association with BBD and one which has not been observed previously despite over 130 years of research on this important pathosystem. One possibility may be that $F$. babinda is insecticolous, interacting with the scale as populations decline and as Neonectria spp. colonize stems, which is likely given this fungus has been recovered from both gypsy moth and hemlock woolly adelgid populations in the eastern United States (Jacobs-Venter et al., 2018). Another plausible, although not mutually exclusive hypothesis based on the observations of sporulation at the base of viable and nonviable perithecia, is that $F$. babinda is a facultative hyperparasite of Neonectria. Clearly, more work is needed to test phytopathogenicity and entomopathogenicity and determine its dominant and facultative lifestyles. Preliminary testing of its ability to degrade tannic acid, a proxy assay for lignin degradation (Kasson et al., 2016) clearly shows that it has strong lignin and cellulose degrading activity.

Finally, in repeated attempts to uncover non-conventional hosts of N. faginata, we discovered a putatively novel Corinectria sp. on red spruce in high elevation sprucefir forests in close proximity to BBD epicenters in VA and WV (Table 1; Figure 3). Interestingly, the fungus appears to be associated with dead trees but the results of those studies and in-depth investigations into the biology and ecology of this putatively 
novel species are forthcoming. However, the Corinectria sp. recovered formed a single clade and was divergent from C. fuckeliana and C. tsugae in the concatenated phylogeny (Figure 4). The third described Corinectria species, C. constricta, was not included in the concatenated phylogeny due to limited availability of sequence data, but based on EF1, isolates recovered in this study were divergent from C. constricta as well. While this appears to be evidence of a novel species of Corinectria, further investigation is needed.

Taken together, the results of this broad study emphasize the sheer diversity of Nectriaceae present in the central Appalachian Mountains. Still, this is undoubtedly a gross underestimation of known and novel fungi as this survey intentionally targeted members of the Nectriaceae with viable and conspicuous perithecia. Exclusively anamorphic fungi were largely excluded with the exception of $F$. babinda. The insights gained here into other fungi of the BBD pathosystem opens many opportunities for further investigation. Specifically, what is the dominant lifestyle of B. ochroleuca, and what role do members of the FBSC actually play in BBD where it appears to dominate tissues recently colonized by scale insects and/or Neonectria fungi? These studies provide an important baseline for how fungal communities assemble, change over time, and affect the forests of the Appalachian Mountains.

\section{Literature Cited}

Barnett, H.L., Lilly, V.G., 1962. A destructive mycoparasite, Gliocladium roseum. 
Mycologia. 54(1), pp.72-77.

Blackburn, L., 2018. [Current distribution of beech bark disease within the United States]. Unpublished raw data.

Booth, C., 1959. Nectria punicea. Mycological Papers 73, 54.

Booth, C., 1967. Nectria galligena. CMI Descript Patho Fungi Bact. 147, 1-2.

Booth, C., 1977. Nectria coccinea var. faginata. C.M.I. Descriptions of Pathogenic Fungi and Bacteria, No. 533 ref.8.

Cale, J.A., Teale, S.A., Johnston, M.T., Boyer, G.L., Perri, K.A., Castello, J.D., 2015. New ecological and physiological dimensions of beech bark disease development in aftermath forests. For. Ecol. Man. 336, 99-108.

Cale, J.A., Garrison-Johnston, M.T., Teale, S.A., Castello, J.D., 2017. Beech bark disease in North America: Over a century of research revisited. For. Ecol. Man. 394, 86-103.

Cale, J.A., Morin, R.S., 2017. County-level detection dates for beech scale in Canada and the United States, https://doi.org/10.7939/DVN/10835, UAL Dataverse, V1.

Carbone I., Kohn L.M., 1999. A method for designing primer sets for speciation studies in filamentous fungi. Mycologia 91, 553-556.

Castlebury, L.A., Rossman, A.Y., Hyten, A. S., 2006. Phylogenetic relationships of Neonectria/Cylindrocarpon on Fagus in North America. Can. J. Bot. 84, 1417-1433.

Chapela, I.H., Boddy, L., 1988. Fungal colonization of attached beech branches. II. 
Spatial and temporal organization of communities arising from latent invaders in bark and functional sapwood, under different moisture regimes. New Phytol. 110, 47-57.

Crane, P.E., Hopkins, A.J.M., Dick, M.A., Bulman, L.S., 2009. Behavior of Neonectria fuckeliana causing a pine canker disease in New Zealand. Can. J. For. Res. 39, 2119-2128.

Ehrlich, J., 1934. The beech bark disease, a Nectria disease of Fagus, following Cryptococcus fagi (Baer.). Can. J. Res. 10, 593-692.

Gräfenhan, T., Schroers, H.J., Nirenberg, H.I., Seifert, K.A., 2011. An overview of the taxonomy, phylogeny, and typification of nectriaceous fungi in Cosmospora, Acremonium, Fusarium, Stilbella, and Volutella. Stud. Mycol., 68, 79-113.

Hewitt, C.G., 1914. Note on the occurrence of the felted beech coccus Cryptococcus fagi (Baerens) Dougl. in Nova Scotia. Can. Entomol. 46, 15-16.

Hendry, S.J., Boddy, L., Lonsdale, D., 2002. Abiotic variable effect differential expression of latent infections in beech (Fagus sylvatica). New Phytol. 155, 449460.

Houston, D.R., Mahoney, E.M., McGauley, B.H., 1987. Beech bark disease: association of Nectria ochroleuca in W.VA., PA, and Ontario. Phytopath. 77, 1615.

Houston, D.R., 1994a. Temporal and spatial shift within the Nectria pathogen complex associated with beech bark disease of Fagus grandifolia. Can. J. For. Res. 24, 960-968.

Houston, D.R. 1994b. Major new tree disease epidemics: beech bark disease. Annu. Rev. Phytopathol. 32,75-87. 
Jager, G., Ten Hoopen, A., Velvis, H., 1979. Hyperparasites of Rhizoctonia solani in Dutch potato fields. Neth. J. Plant Pathol. 85, 253-268.

Jacobs-Venter, A., Laraba, I., Geiser, D.M., Busman, M., Vaughan, M.M., Proctor, R.H., McCormick, S.P., O'Donnell, K., 2018. Molecular systematics of two sister clades, the Fusarium concolor and F. babinda species complexes, and the discovery of a novel microcycle macroconidium-producing species from South Africa. Mycologia. 110, 1189-1204.

Kasson, M.T., Livingston, W.H. 2009. Spatial distribution of Neonectria species associated with beech bark disease in northern Maine. Mycologia, 101, 190-195.

Katoh K., Standley D.M., 2013. MAFFT multiple sequence alignment software version 7: improvements in performance and usability. Mol. Biol. Evol. 30, 772-780.

Landan G., Graur D., 2008. Local reliability measures from sets of co-optimal multiple sequence alignments. Pacific Symposium on Biocomputing 13, 15-24.

Lohman, M.L., Watson, A.J., 1943. Identity and host relations of Nectria species associated with diseases of hardwoods in the United States. Lloydia, 6, 77-108.

Lortie, M., Kuntz, J. E., 1963. Ascospore discharge and conidium release by Nectria galligena Bres. under field and laboratory conditions. Can. J. Bot. 41, 1203-1210. Macias, A.M., Geiser, D.M., Stajich, J.E., Lukasik, P., Veloso, C., Bublitz, D.C., Berger, M.C., Boyce, G.R., Hodge, K. and Kasson, M.T., 2019. Evolutionary relationships among Massospora spp.(Entomophthorales), obligate pathogens of cicadas. Mycologia.

Mielke, M.E., Haynes, C., MacDonald, W. L., 1982. Beech scale and Nectria galligena 
on beech in the Monongahela National Forest, West Virginia. Plant Dis. 66, 851852.

Mielke, M.E. and Houston, D.R., 1983. Beech bark disease in West Virginia: status and impact on the Monongahela National Forest. In In: Proceedings, IUFRO Beech Bark Disease Working Party Conference; 1982 September 26-October 8; Hamden, CT. Sponsored by the USDA Forest Service, Northeastern Forest Experiment Station. Gen. Tech. Rep. WO-37. [Washington, DC]: US Department of Agriculture, Forest Service: 27-30.

Morin, R.S., Liebhold, A.M., Tobin, P.C., Gottschalk, K.W., Luzader, E., 2007. Spread of beech bark disease in the eastern United States and its relationship to regional forest composition. Can. J. For. Res. 37, 726-736.

O'Donnell, K., Cigelnik, E., 1997. Two divergent intragenomic rDNA ITS2 types within a monophyletic lineage of the fungus Fusarium are nonorthologous. Mol. Phylogenet. Evol. 7, 103-116.

Plante, F., Bernier, L., 1997. Variability of virulence of Nectria galligena towards northern hardwoods. Eur. J. For. Path. 27, 261-272.

R Core Team., 2020. R: a language and environment for statistical computing. $R$ Foundation for Statistical Computing, Vienna, Austria.

Rambaut, A., 2017. FigTree-version 1.4.3, a graphical viewer of phylogenetic trees.

Rambaut, A., Drummond, A.J., Xie, D., Baele, G., Suchard, M.A., 2018. Posterior summarization in Bayesian phylogenetics using Tracer 1.7. Syst. Biol. 67, 901904.

Rehner, S., 2001. Primers for Elongation Factor 1- $\alpha$ (EF1- $\alpha)$. Insect Biocontrol 
Laboratory: USDA, ARS, PSI.

Rehner, S.A., Samuels, G.J., 1994. Taxonomy and phylogeny of Gliocladium analyzed from nuclear large subunit ribosomal DNA sequences. Mycol. Res. 98, 625-634.

Ronquist, F., Teslenko, M., Van Der Mark, P., Ayres, D.L., Darling, A., Höhna, S., Larget, B., Liu, L., Suchard, M.A., Huelsenbeck, J.P., 2012. MrBayes 3.2: efficient Bayesian phylogenetic inference and model choice across a large model space. Syst. Biol. 61, 539-542.

Sela, I., Ashkenazy, H., Katoh, K., Pupko, T., 2015. GUIDANCE2: accurate detection of unreliable alignment regions accounting for the uncertainty of multiple parameters. Nucleic Acids Res., 43, W7-W14.

Spaulding, P., Grant, T.J., Ayers, T.T., 1936. Investigations of Nectria diseases in hardwoods of New England. J. For. 34, 169-179.

Stecher, G., Tamura, K., Kumar, S., 2020. Molecular Evolutionary Genetics Analysis (MEGA) for macOS. Mol. Biol. Evol.

Thomsen, M., Buchwald, N.F., Hauberg, P., 1949. Angreb af Cryptococcus fagi, Nectria galligena og andre parasiter paa bog i Danmark 1939-43. Det Forstlige Forsogsvaesen, 18, 97-326.

Turhan, G., 1993. Mycoparasitism of Alternaria alternata by an additional eight fungi indicating the existence of further unknown candidates for biological control. J. Phytopathol., 138, 283-292.

Vilgalys, R., Hester, M., 1990. Rapid genetic identification and mapping of enzymatically amplified ribosomal DNA from several Cryptococcus species. J. Bacteriol. 172, 4238-4246. 
Villesen, P., 2007. FaBox: an online toolbox for fasta sequences. Mol. Ecol.

Notes, 7, 965-968.

Welch, D.S., 1934. The range and importance of Nectria canker on hardwoods in the Northeast. J. For. 32, 997-1002.

White, T.J., Bruns, T., Lee, S., Taylor, J. 1990. Amplification and direct sequencing of fungal ribosomal RNA genes for phylogenetics. PCR Protocol. Guide Methods. Appl. 18, 315-322.

\section{Supplemental Figures}

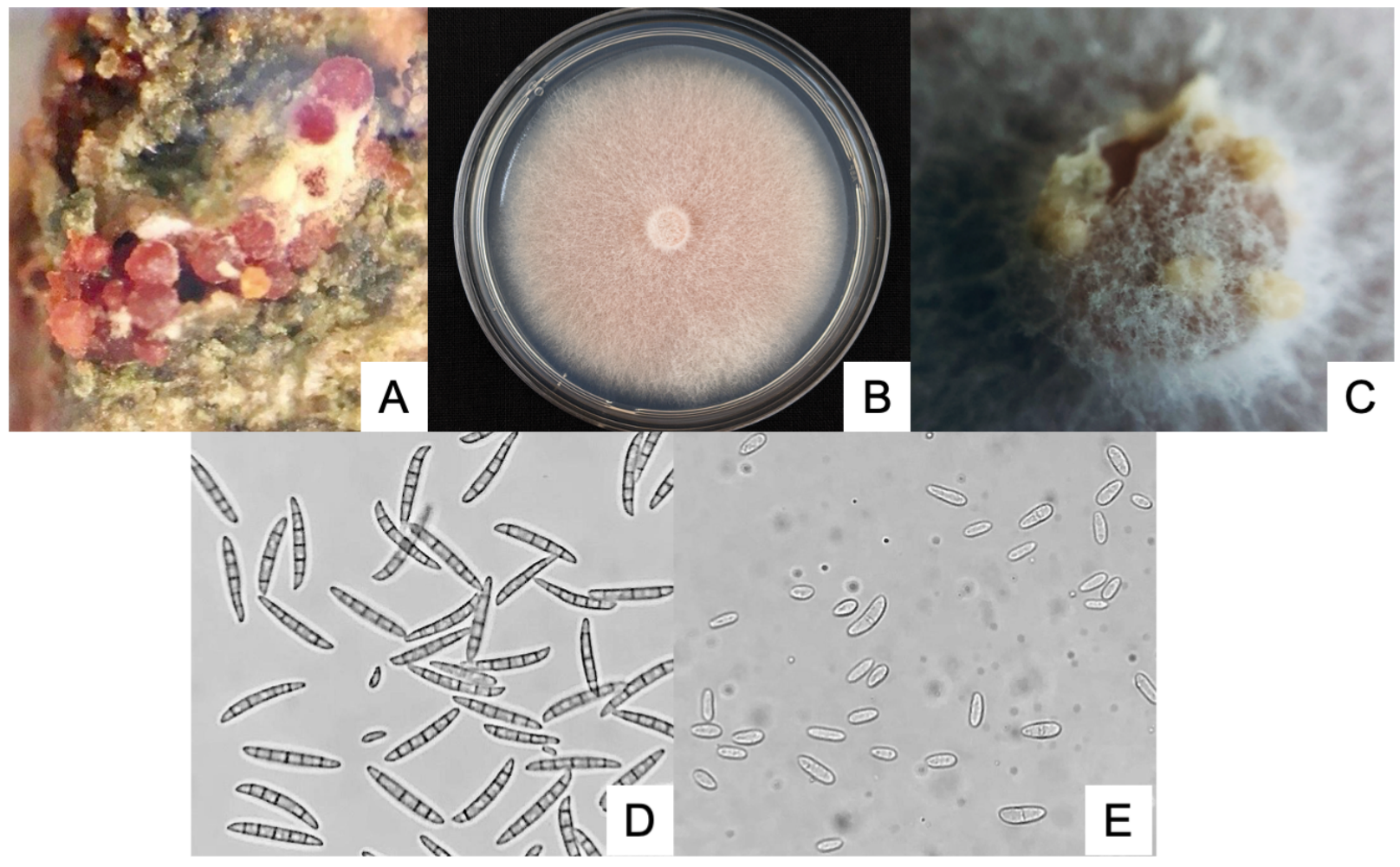

Supplemental Figure 1: Morphological characteristics of Fusarium babinda: A) F. babinda sporulation on Neonectria sp. perithecia; B) 7-day old culture on PDA; B) sporodochia bearing macro- and micro-conidia; C) macroconidia; D) microconidia 


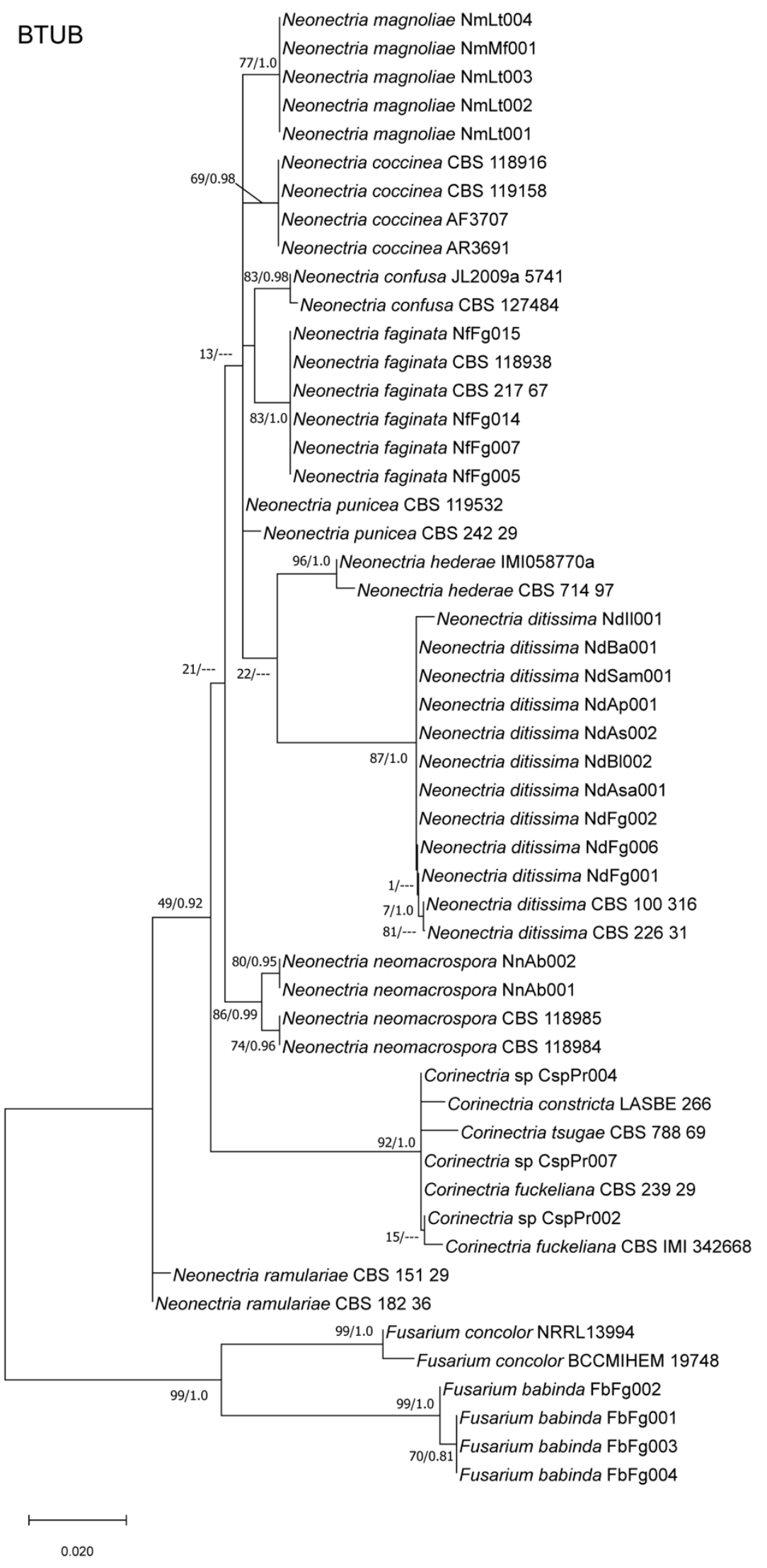




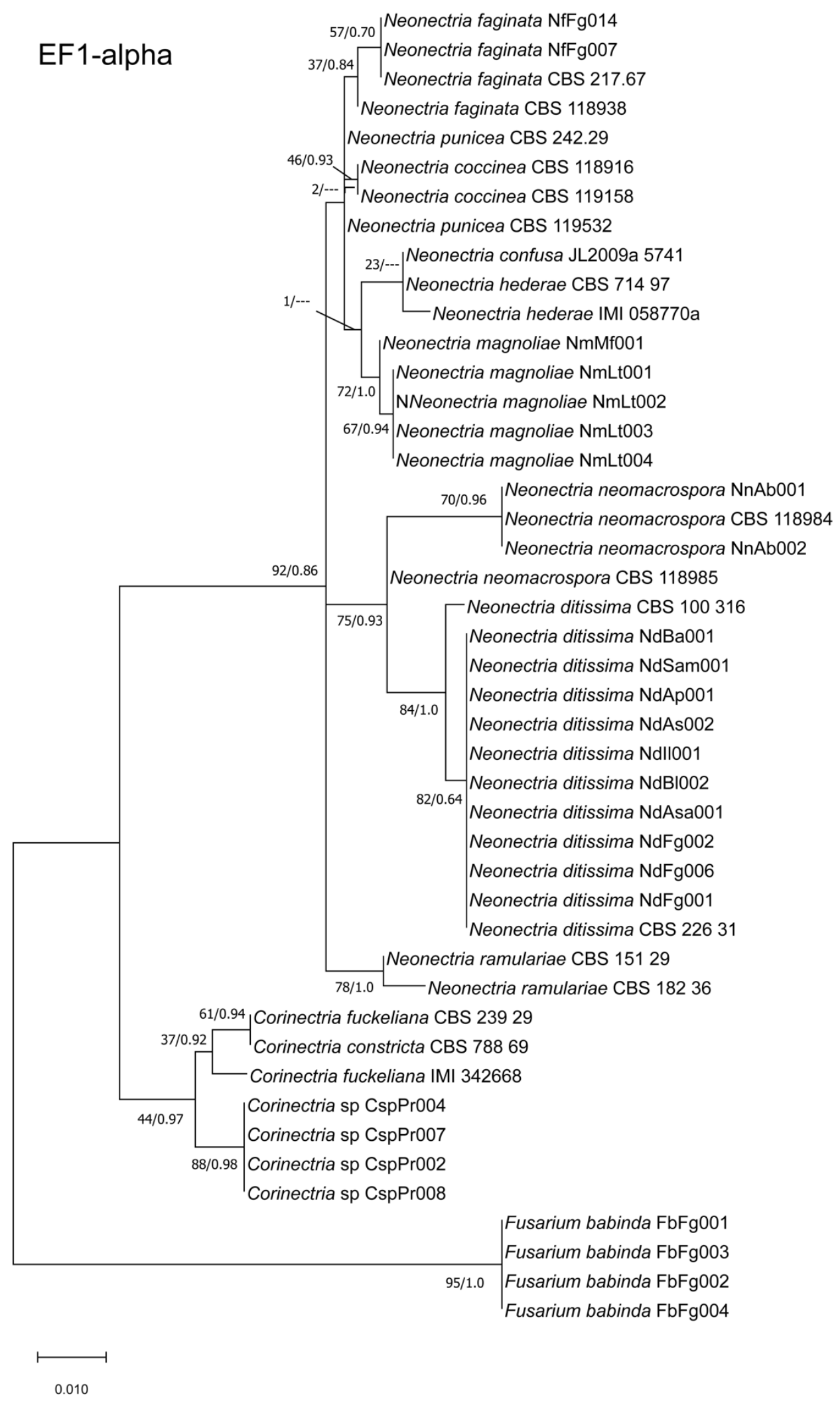


ITS

68/0.98 Neonectria neomacrospora CBS 118984

79/1.0 Neonectria neomacrospora CBS 118985

Neonectria neomacrospora NnAb002

Neonectria neomacrospora NnAb001

Neonectria ditissima NdII001

Neonectria ditissima NdFg001

- Neonectria ditissima $\mathrm{NdBa001}$

Neonectria ditissima NdFg002

40/0.99 Neonectria ditissima NdFg006

Neonectria ditissima NdAp001

Neonectria ditissima $\mathrm{NdBI} 002$

Neonectria ditissima CBS 100.316

Neonectria ditissima CBS 226.31

Neonectria ditissima NdSam001

Neonectria ditissima NdAsa001

Neonectria ditissima NdAs002

$90 / 0.99$

2/--

Neonectria ramulariae CBS15129

Neonectria ramulariae CBS 18236

-Neonectria hederae CBS 71497

Corinectria sp CspPr007

26/--- Corinectria sp CspPr004

40/1.0 Neonectria coccinea CBS118916

Neonectria coccinea CBS 119158

Neonectria coccinea AR3691

Neonectria coccinea AF3707

Neonectria magnoliae NmMf001

Neonectria magnoliae NmLt004

1/--- Neonectria magnoliae NmLt003

$1 /-\cdot$

Neonectria magnoliae NmLt002

Neonectria magnoliae NmLt001

-Neonectria punicea CBS 119532

Corinectria tsugae CBS 788.69

26/1.0 | $\begin{aligned} & \text { Corinectria fuckeliana IMI } 342668 \\ & \text { Corinectria fuckeliana CBS } 239.29\end{aligned}$

$21 / 0.87$

Corinectria sp CspPr008

31/0.96 Corinectria sp CspPr002

Neonectria confusa JL 2009a 5741

- Neonectria confusa CBS 127484

$14 /---$

Neonectria faginata NfFg014

Neonectria faginata NfFg015

Neonectria faginata NfFg007

$93 / 1.0$

Neonectria faginata CBS 21767

Neonectria faginata CBS 118938

Neonectria faginata NfFg005

Neonectria punicea CBS 24229

Fusarium babinda FbFg002

Fusarium babinda $\mathrm{FbFg003}$

Fusarium babinda FbFg001

-Fusarium babinda FbFg004

42/---

Fusarium concolor NRRL13994

99/1.0 Fusarium concolor BCCMIHEM19748

0.050 


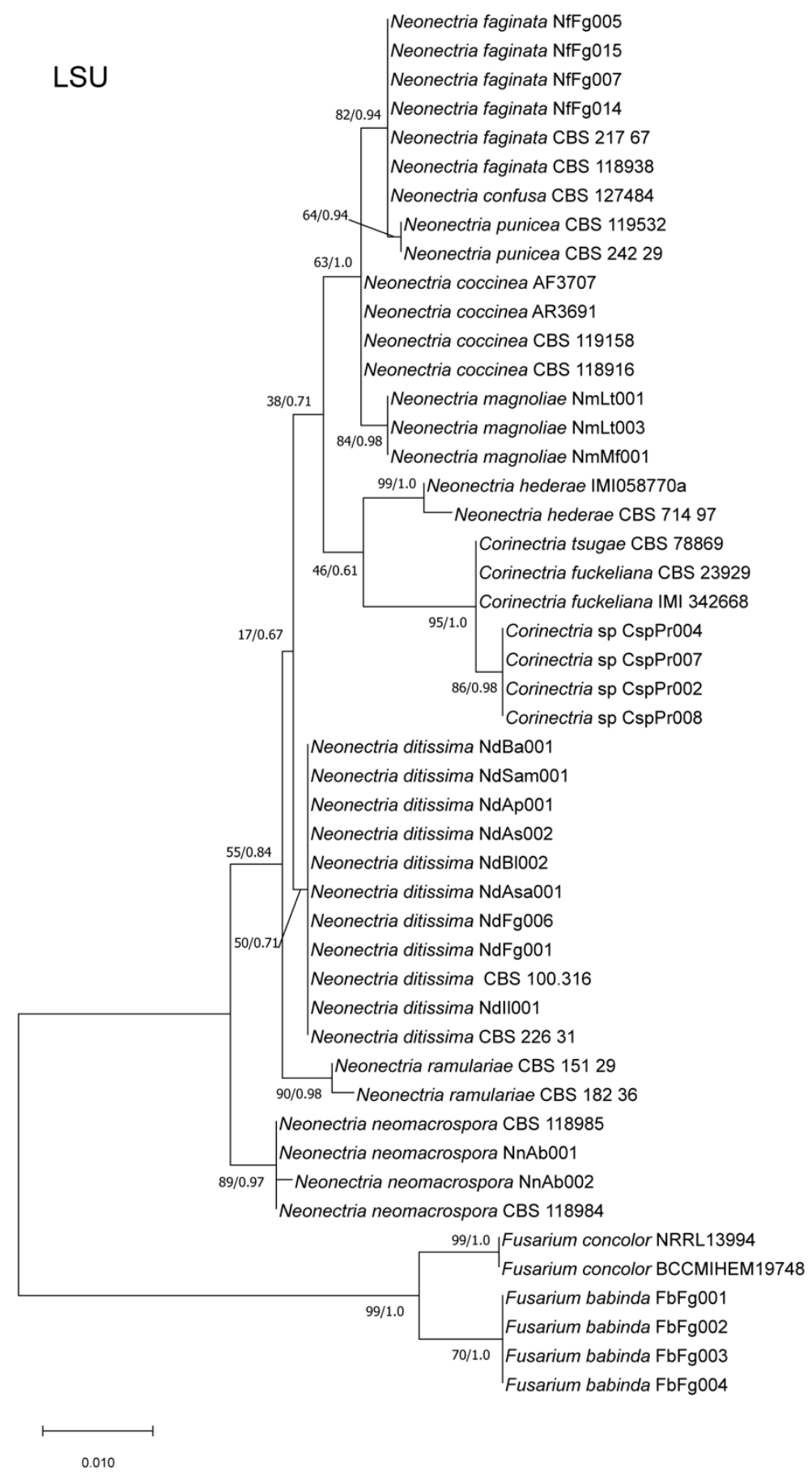

Supplemental Figures 2 - 5: Single-gene (BTUB, EF1, ITS, LSU) phylogenetic tree of Neonectria spp., Corinectria spp. and outgroups. Topology and branch lengths are from the $M L$ analysis. For each node supported in the $M L$ analysis, bootstrap support and posterior probabilities are indicated (ML/BI). 


\section{Supplemental Tables}

Supplemental Table 1: Locations of sampling sites (including areas within these sites if applicable). The presence of BBD in each site is noted. Additionally, the general coordinates of the sampling locations are provided.

\begin{tabular}{|c|c|c|c|}
\hline Site & Location & BBD Present & Coordinates \\
\hline $\mathrm{DM}$ & Allegany County, MD & $\mathrm{Y}$ & $(39.562432,-78.948432)$ \\
\hline PS & Allegany County, MD & $\mathrm{Y}$ & $(39.34755,-79.27962)$ \\
\hline SM1 & Cherokee County, NC & $\mathrm{Y}$ & $(35.610143,-83.421962)$ \\
\hline SM2 & Cherokee County, NC & $\mathrm{Y}$ & $(35.598317,-83.42741)$ \\
\hline SMR & Cherokee County, NC & $\mathrm{Y}$ & $(35.5959,-83.460056)$ \\
\hline SM3 & Cherokee County, NC & $\mathrm{Y}$ & $(35.631206,-83.467966)$ \\
\hline $\mathrm{RH}$ & Mitchell County, NC & $\mathrm{Y}$ & $(36.09984,-82.09217)$ \\
\hline PM & Fayette County, PA & $\mathrm{N}$ & $(39.740354,-79.89975)$ \\
\hline MR & Grayson County, VA & $\mathrm{Y}$ & $(36.657625,-81.520676)$ \\
\hline WT & Grayson County, VA & $\mathrm{Y}$ & $(36.633437,-81.60132)$ \\
\hline BG & Monongalia County, WV & $\mathrm{Y}$ & $(39.626579,-79.864538)$ \\
\hline $\mathrm{SH}$ & Monongalia County, WV & $\mathrm{N} / \mathrm{A}$ & $(39.614379,-79.829591)$ \\
\hline UF & Monongalia County, WV & $\mathrm{N} / \mathrm{A}$ & $(39.669081,-79.771688)$ \\
\hline AR & Monongalia County, WV & $\mathrm{N}$ & $(39.644653,-79.976096)$ \\
\hline MON & Monongalia County, WV & $\mathrm{N} / \mathrm{A}$ & $(39.619082,-79.957879)$ \\
\hline UW & Monongalia County, WV & $\mathrm{N}$ & $(39.66875,-79.934172)$ \\
\hline SK & Pendleton County, WV & $\mathrm{Y}$ & $(38.699077,-79.533215)$ \\
\hline GK1 & Randolph County, WV & $\mathrm{Y}$ & $(38.60741,-79.84431)$ \\
\hline GK2 & Randolph County, WV & $\mathrm{Y}$ & $(38.61129,-79.84167)$ \\
\hline GK3 & Randolph County, WV & $\mathrm{Y}$ & $(38.61768,-79.84119)$ \\
\hline GK5 & Randolph County, WV & $\mathrm{Y}$ & $(38.62928,-79.8429)$ \\
\hline GK6 & Randolph County, WV & $\mathrm{Y}$ & $(38.65584,-79.83899)$ \\
\hline GKT & Randolph County, WV & $\mathrm{Y}$ & $(38.614709,-79.842769)$ \\
\hline 92 & Randolph County, WV & $\mathrm{Y}$ & $(38.66397,-79.90788)$ \\
\hline BM & Randolph County, WV & $\mathrm{Y}$ & $(38.61641,-79.72984)$ \\
\hline 227 & Randolph County, WV & $\mathrm{Y}$ & $(38.62952,-79.927647)$ \\
\hline NB & Ritchie County, WV & $\mathrm{N}$ & $(39.216486,-81.09951)$ \\
\hline $\mathrm{FN}$ & Tucker County, WV & $\mathrm{N} / \mathrm{A}$ & $(39.034,-79.685)$ \\
\hline MM1 & Yancey County, WV & $\mathrm{Y}$ & $(35.71076,-82.24445)$ \\
\hline MM2 & Yancey County, WV & $\mathrm{Y}$ & $(35.724152,-82.281468)$ \\
\hline
\end{tabular}


Supplemental Table 2: Primer sequences, PCR parameters, and sources for each primer pair used to generate sequence data.

\begin{tabular}{|c|c|c|c|c|c|c|c|c|c|c|c|c|c|c|}
\hline $\begin{array}{l}\text { Target } \\
\text { Locus }\end{array}$ & Primer & Primer Sequence & \multicolumn{2}{|c|}{$\begin{array}{c}\text { Initial } \\
\text { Denature }\end{array}$} & Cycles & \multicolumn{2}{|c|}{ Denature } & \multicolumn{2}{|c|}{ Anneal } & \multicolumn{2}{|c|}{ Extension } & \multicolumn{2}{|c|}{$\begin{array}{c}\text { Final } \\
\text { Extension }\end{array}$} & Source \\
\hline \multirow{2}{*}{ ITS } & ITS5 & GGAAGTAAAAGTCGTAACAAGG & \multirow{2}{*}{$95 \mathrm{C}$} & \multirow{2}{*}{$2: 00$} & \multirow{2}{*}{35} & \multirow{2}{*}{$95 \mathrm{C}$} & \multirow{2}{*}{$0: 30$} & \multirow{2}{*}{$56 \mathrm{C}$} & \multirow{2}{*}{$0: 30$} & \multirow{2}{*}{$72 \mathrm{C}$} & \multirow{2}{*}{$1: 00$} & \multirow{2}{*}{$72 \mathrm{C}$} & \multirow{2}{*}{$7: 00$} & \multirow{2}{*}{ White et al., 1990} \\
\hline & ITS4 & TCCTCCGCTTATTGATATGC & & & & & & & & & & & & \\
\hline \multirow{2}{*}{ tef-1a } & EF1-728 & CATCGAGAAGTTCGAGAAGG & \multirow{2}{*}{$95 \mathrm{C}$} & \multirow{2}{*}{ 2:00 } & \multirow{2}{*}{35} & \multirow{2}{*}{$95 \mathrm{C}$} & \multirow{2}{*}{$0: 30$} & \multirow{2}{*}{$56 \mathrm{C}$} & \multirow{2}{*}{$0: 30$} & \multirow{2}{*}{$72 \mathrm{C}$} & \multirow{2}{*}{$1: 00$} & \multirow{2}{*}{$72 \mathrm{C}$} & \multirow{2}{*}{ 7:00 } & $\begin{array}{c}\text { Carbone and } \\
\text { Kohn, } 1999\end{array}$ \\
\hline & EF1-1576 & ACHGTRCCRATACCACCRATCTT & & & & & & & & & & & & Rehner 2001 \\
\hline nrLSU & $\begin{array}{l}\text { LR5 } \\
\text { LROR }\end{array}$ & $\begin{array}{c}\text { ATCCTGAGGGAAACTTC } \\
\text { GTACCCGCTGAACTTAAGC }\end{array}$ & $95 \mathrm{C}$ & $2: 00$ & 35 & $95 \mathrm{C}$ & $0: 30$ & $51.1 \mathrm{C}$ & $0: 45$ & $72 \mathrm{C}$ & $1: 30$ & $72 \mathrm{C}$ & $7: 00$ & $\begin{array}{l}\text { Vilgalys and } \\
\text { Hester, } 1990 \\
\text { Rehner and } \\
\text { Samuels, } 1994\end{array}$ \\
\hline Beta- & $\mathrm{T} 1$ & AACATGCGTGAGATTGTAAGT & $95 \mathrm{C}$ & $2 \cdot 00$ & 35 & $95 \mathrm{C}$ & 0.30 & $64 \mathrm{C}$ & 0.30 & $72 \mathrm{C}$ & $1: 00$ & $72 \mathrm{C}$ & $7 \cdot 00$ & O'Donnell and \\
\hline tublin & $\mathrm{T} 2$ & TAGTGACCCTTGGCCCAGTTG- & & & & & & & & & & & & Cigelnik, 1997 \\
\hline
\end{tabular}


Supplemental Table 3: All BLASTn results from representative isolates recovered in this survey. The accession number, query coverage, and percent identity are provided for the top hit for each sequence.

\begin{tabular}{|c|c|c|c|c|}
\hline \multirow{2}{*}{ Isolate } & \multirow{2}{*}{ Closest Match } & \multirow{2}{*}{ Accession } & \multicolumn{2}{|r|}{ ITS } \\
\hline & & & $\%$ ID & Query Cover (\%) \\
\hline F10121 & Nectria magnoliae & NR_160076.1 & 100 & 100 \\
\hline F10222 & Nectria magnoliae & NR_160076.1 & 99.8 & 78 \\
\hline F10511 & Nectria magnoliae & NR_160076.1 & 98.38 & 77 \\
\hline F10521 & Nectria magnoliae & NR_160076.1 & 99.2 & 81 \\
\hline F10522 & Nectria magnoliae & NR_160076.1 & 99.6 & 100 \\
\hline AM52 & Nectria magnoliae & NR_160076.1 & 99 & 94 \\
\hline AM57 & Nectria magnoliae & NR_160076.1 & 98.39 & 99 \\
\hline Neomag21 & Nectria magnoliae & NR_160076.1 & 99.4 & 98 \\
\hline Neomag32 & Nectria magnoliae & NR_160076.1 & 99.6 & 100 \\
\hline GKMAG1 & Nectria magnoliae & NR_160076.1 & 98.6 & 83 \\
\hline GKMAG2 & Nectria magnoliae & NR_160076.1 & 99.6 & 86 \\
\hline BG1423 & Neonectria faginata & KR019361.1 & 99.6 & 100 \\
\hline GK311 & Neonectria faginata & KR019361.1 & 99.4 & 99 \\
\hline SM223 & Neonectria faginata & KR019361.1 & 99.02 & 96 \\
\hline SM225 & Neonectria faginata & KR019361.1 & 99.2 & 100 \\
\hline DM731 & Neonectria faginata & KR019361.1 & 100 & 96 \\
\hline DM1_FCSC & Fusarium babinda & NR_159861.1 & 99.8 & 100 \\
\hline PSF164 & Fusarium babinda & NR_159861.1 & 100 & 99 \\
\hline BG10a & Fusarium sp. & MK102657.1 & 99.8 & 96 \\
\hline GK554 & Fusarium babinda & NR_159861.1 & 99.8 & 94 \\
\hline GK581 & Neonectria ditissima & MN̄843954.1 & 98.9 & 98 \\
\hline UFBEAL & Neonectria ditissima & JF735309.1 & 98.55 & 100 \\
\hline SOAMRED2 & Neonectria ditissima & JF735309.1 & 99.1 & 100 \\
\hline SOAMRED5 & Neonectria ditissima & JF735309.1 & 99.7 & 100 \\
\hline MM2AS512 & Neonectria ditissima & MN843954.1 & 99.7 & 100 \\
\hline MM2AS614 & Neonectria ditissima & MN843954.1 & 99.7 & 100 \\
\hline RHS142 & Neonectria ditissima & JF735309.1 & 99.7 & 100 \\
\hline SM214 & Neonectria ditissima & JF735309.1 & 99.7 & 100 \\
\hline SMRAS22 & Neonectria ditissima & JF735309.1 & 99.7 & 100 \\
\hline SMRAS25 & Neonectria ditissima & JF735309.1 & 99.4 & 100 \\
\hline SMRAS3w & Neonectria ditissima & JF735309.1 & 99.7 & 100 \\
\hline SMRAS3w5 & Neonectria ditissima & JF735309.1 & 98.7 & 99 \\
\hline WTS125 & Neonectria ditissima & MN843954.1 & 99.7 & 100 \\
\hline SKI12A & Neonectria ditissima & JF735309.1 & 99.23 & 96 \\
\hline
\end{tabular}




\begin{tabular}{|c|c|c|c|c|}
\hline SKI31A & Neonectria ditissima & JF735309.1 & 98.29 & 94 \\
\hline SKI23A & Neonectria ditissima & MN843954.1 & 99.1 & 93 \\
\hline SKI31B & Neonectria ditissima & JF735309.1 & 99.01 & 89 \\
\hline MM2S313y & Cosmospora obscura & KC291719.1 & 100 & 99 \\
\hline MM2S31p & Cosmospora obscura & KC291719.1 & 99.04 & 99 \\
\hline GK61053 & Bionectria ochroleuca & HQ328025.1 & 98.85 & 100 \\
\hline SM2TIAM3 & Bionectria ochroleuca & HQ328025.1 & 98.5 & 100 \\
\hline MM2AS113 & Thelonectria veuillotiana & JQ734922.1 & 99.64 & 100 \\
\hline MM2AS115 & Thelonectria veuillotiana & JQ734922.1 & 99.8 & 99 \\
\hline MM2AS211 & Thelonectria veuillotiana & JQ734922.1 & 99.64 & 99 \\
\hline MM2AS215 & Thelonectria veuillotiana & JQ734922.1 & 100 & 97 \\
\hline MR12 & Corinectria fuckeliana & MH859658.1 & 99.1 & 100 \\
\hline PIRUPERI & Corinectria fuckeliana & MF593148.1 & 95.7 & 100 \\
\hline PIRUSCR1 & Corinectria fuckeliana & MF593148.1 & 97.04 & 93 \\
\hline PIRUSCR2 & Corinectria fuckeliana & MF593148.1 & 98.8 & 94 \\
\hline WTPIRU4 & Corinectria fuckeliana & MF593148.1 & 94.8 & 99 \\
\hline MR13ODD & $\begin{array}{c}\text { Neonectria } \\
\text { neomacrospora }\end{array}$ & MH580206.1 & 99.21 & 93 \\
\hline MR130DDA & $\begin{array}{c}\text { Neonectria } \\
\text { neomacrospora }\end{array}$ & MH580206.1 & 99.21 & 93 \\
\hline ABBA1N2 & Thyronectria balsamea & JN995618.1 & 99.64 & 97 \\
\hline ABBA1N1 & Thyronectria balsamea & JN995618.1 & 99.44 & 73 \\
\hline \multirow{2}{*}{ Isolate } & \multirow{2}{*}{ Closest Match } & \multirow{2}{*}{ Accession } & \multicolumn{2}{|r|}{ RPB2 } \\
\hline & & & $\%$ ID & Query Cover (\%) \\
\hline AM57 & Nectria magnoliae & HQ897713 & 99.77 & 91 \\
\hline F10521 & Nectria magnoliae & HQ897713 & 99.9 & 89 \\
\hline F10522 & Nectria magnoliae & HQ897713 & 99.1 & 90 \\
\hline
\end{tabular}


Supplemental Table 4: Recovery of Fusarium babinda isolates from bark tissue samples collected from asymptomatic American beech trees (shaded grey) or American beech trees with signs or symptoms associated with beech bark disease. Values represent the number of bark samples yielding Fusarium babinda at the designated sampling location.

\begin{tabular}{|c|c|c|c|c|c|c|c|c|}
\hline Site & $\begin{array}{c}\text { Trees } \\
\text { Sampled }\end{array}$ & Host & $\begin{array}{c}\text { Total Bark } \\
\text { Samples }\end{array}$ & $\begin{array}{c}\text { Scale } \\
\text { Present }\end{array}$ & $\begin{array}{c}\text { Perithecia } \\
\text { Present }\end{array}$ & N. faginata & N. ditissima & F. babinda \\
\hline ARB & 12 & FAGR & 96 & $\mathrm{~N}$ & $\mathrm{~N}$ & - & - & - \\
\hline BG & 4 & FAGR & 32 & $\mathrm{~N}$ & $\mathrm{~N}$ & - & - & - \\
\hline $\mathrm{FH}$ & 12 & FAGR & 96 & $\mathrm{~N}$ & $\mathrm{~N}$ & - & - & - \\
\hline UWL & 15 & FAGR & 120 & $\mathrm{~N}$ & $\mathrm{~N}$ & - & - & - \\
\hline NBB & 12 & FAGR & 96 & $\mathrm{~N}$ & $\mathrm{~N}$ & - & - & - \\
\hline BG & 5 & FAGR & 96 & $Y$ & Y & - & - & 27 \\
\hline $\mathrm{DM}$ & 5 & FAGR & 60 & $Y$ & $Y$ & 6 & - & 21 \\
\hline GK & 25 & FAGR & 248 & $Y$ & $Y$ & 30 & - & 83 \\
\hline PSF & 7 & FAGR & 72 & Y & Y & - & - & 29 \\
\hline $\mathrm{MM}$ & 5 & FAGR & 40 & $Y$ & $Y$ & 2 & - & 2 \\
\hline MR & 2 & FAGR & 12 & Y & $Y$ & - & - & 1 \\
\hline PSF & 7 & FAGR & 72 & $Y$ & $Y$ & - & - & 29 \\
\hline SK & 2 & FAGR & 36 & $Y$ & $Y$ & 28 & - & 3 \\
\hline SM & 14 & FAGR & 112 & $Y$ & $Y$ & 5 & - & 44 \\
\hline WT & 1 & FAGR & 8 & $Y$ & $Y$ & - & - & 1 \\
\hline
\end{tabular}




\section{CHAPTER 2: Characterization of mating type genes in heterothallic Neonectria species with emphasis on $\mathbf{N}$. coccinea, $\boldsymbol{N}$. ditissima, and $\boldsymbol{N}$. faginata}

\section{Abstract}

Neonectria ditissima and N. faginata are canker pathogens involved in an insectfungus disease complex of American beech (Fagus grandifolia) commonly known as beech bark disease (BBD). In Europe, both $N$. ditissima and $N$. coccinea are involved in BBD on European beech (Fagus sylvatica). Field observations across the range of BBD indicate that new infections occur primarily via ascospores. Both heterothallic (selfsterile) and homothallic (self-fertile) mating strategies have been reported for Neonectria fungi. As such, investigations into mating strategy are important for understanding both the disease cycle and population genetics of Neonectria. This is particularly important in the U.S. given that over time N. faginata dominates the BBD pathosystem despite high densities of non-beech hosts for $N$. ditissima. This study utilized whole-genome sequences of BBD-associated Neonectria spp. along with other publicly available Neonectria and Corinectria genomes and in vitro mating assays to characterize mating type (MAT) loci and confirm thallism for select members of Neonectria and Corinectria. MAT gene-specific primer pairs were developed to efficiently characterize the mating types of additional single ascospore strains of $N$. ditissima, $N$. faginata, and $N$. coccinea and several other related species lacking genomic data. In vitro mating assays were used in combination with molecular results to confirm thallism. These assays also confirmed the sexual compatibility among $N$. ditissima strains from different plant hosts. Maximum likelihood phylogenetic analysis of both MAT1-1-1 and MAT1-2-1 sequences 
recovered trees with similar topology to previously published phylogenies of Neonectria and Corinectria. The results of this study indicate that all Neonectria and Corinectria tested are heterothallic based on the limited sampling and, as such, thallism cannot help explain the inevitable dominance of $N$. faginata in the BBD pathosystem.

\section{Introduction}

Beech bark disease (BBD), a canker disease complex arising from interactions among insect and fungal causal agents, has significantly impacted the health of American (Fagus grandifolia Ehrh.) and European (Fagus sylvatica L.) beech forests throughout North America and Europe over the last century (Cale et al., 2017; Ehrlich, 1934; Thomsen et al., 1949). More recently, BBD has intensified in areas where historic cold temperatures have kept the disease in check, raising concerns for the impact that global climate change may have on the expansion of this disease and other plant pathogens (Dukes et al., 2009; Kasson and Livingston, 2012; McCullough and Wieferich, 2001; McLaughlin and Greifenhagen, 2012).

Beech bark disease requires prior infestation by a non-native scale insect Cryptococcus fagisuga Lind., which predisposes the host's bark tissues to subsequent invasion by one or more closely related canker fungi: Neonectria ditissima ([Tul. \& C. Tul.] Samuels \& Rossman), N. faginata ([Lohman, Watson, \& Ayres] Castl. \& Watson), and N. coccinea ([Pers.] Rossman and Samuels) (Houston, 1994b; Thomsen et al., 1949). Neonectria ditissima (formerly N. galligena Bres.) has been implicated in BBD both in the U.S. and in Europe while N. faginata (formerly N. coccinea var. faginata (Pers.:Fr.) Fr. Var. Lohman, A. M. Watson, \& Ayers) appears restricted to American beech in the U.S. and N. coccinea to European beech in Europe (Thomsen et al., 1949; 
Castlebury et al., 2006). Bionectria ochroleuca ([Schwein.] Schroers \& Samuels) has also been implicated in the U.S., but its role in BBD is not well understood (Houston et al., 1987).

Neonectria ditissima is perhaps best known as the causal agent of perennial target canker on many non-beech hosts including birch, maple, and walnut among others (Lohman and Watson, 1943; Spaulding et al., 1936; Booth, 1967). Neonectria faginata is unique to the BBD pathosystem in that it has only been observed causing annual cankers on American beech trees following C. fagisuga infestation (Castlebury et al., 2006). Other native plant hosts of $N$. faginata have not been detected. Unlike $N$. ditissima and $N$. faginata, $N$. coccinea is known to persist endophytically (asymptomatically) in the bark of European beech, with the ability to initiate disease following wounding, including but not limited to damage inflicted by C. fagisuga (Chapela and Boddy, 1988; Hendry et al., 2002). When present, fruiting structures of Neonectria species are easily recognizable as bright red or orange, globose sexual ascocarps (perithecia) bearing uniseptate, hyaline ascospores. While perithecia are often products of mating between two distinct thalli of the opposite mating type, selffertility (homothallism) can lead to the completion of the sexual cycle through selfing, which has been previously confirmed for members of the Nectriaceae (Alexopolous et al., 1996; Yun et al., 2000).

Sexual reproduction in ascomycetes is generally understood to be regulated by the presence of one or both mating-type (MAT) idiomorphs (MAT1-1 and MAT1-2) at a mating type locus (Coppin et al., 1997; Turgeon, 1998). The term "idiomorph" refers to sequences which encode different functional proteins but are found occupying the same 
locus in different strains. For heterothallic ascomycetes, three genes (MAT1-1-1, MAT11-2, and MAT1-1-3) are commonly found at the MAT locus for the MAT1-1 mating type while two genes (MAT1-2-1 and MAT1-2-2) often occur at this locus for the MAT1-2 mating type (Coppin et al., 1997; Pöggeler and Kück, 2000).

These mating type idiomorphs encode polypeptides responsible for the regulation of the sexual mating cycle in filamentous fungi (Kronstad and Staben, 1997). For the MAT1-1 idiomorph, MAT1-1-1, MAT1-1-2, and MAT1-1-3 encode proteins with an a domain, a PPF domain, and an HMG (high-mobility group) domain, respectively (Debuchy et al., 2010). Of these, the $\alpha$ domain protein encoded by MAT1-1-1 is responsible for MAT identity and sexual development (Saupe et al., 1996). The PPF and HMG domain proteins appear to have redundant roles in fertility as the deletion of one or the other has no apparent effect, while the deletion of both has been shown to decrease fertility (Ferreira et al., 1998).

For the MAT1-2 idiomorph, MAT1-2-1 encodes a protein with an HMG domain responsible for the establishment of MAT identity (Chang and Staben, 1994; Coppin et al., 1997). MAT1-2-2 encodes a small open reading frame (ORF) that does not have an apparent function (Pöggeler and Kück, 2000), but this ORF appears to be absent in some filamentous fungi (Debuchy and Coppin, 1992). Both MAT proteins act as transcription factors and are required for the initiation and regulation of the sexual cycle. Self-fertile (homothallic) fungi contain both MAT genes critical for sexual development (MAT1-1-1 and MAT1-2-1) at the mating type locus. In this case, reliance on a complementary mating type to complete the sexual cycle is not required. 
Both homothallism and heterothallism have been previously reported for $N$. ditissima (El-Gholl et al., 1986; Krüger, 1973) while only heterothallism has been reported for $N$. faginata (Cotter and Blanchard, 1978). However, these determinations relied solely on culture-based observations via in vitro mating assays. No molecular characterization of the MAT locus has been completed for any member of Neonectria despite the publicly available genomes of several species in the genus (SalgadoSalazar and Crouch, 2019; Gómez-Cortecero et al., 2015; Deng et al., 2015).

Confirming the thallism of the BBD pathogens is important for several reasons: 1) thallism is predicted to affect expected patterns of genomic diversity via obligate or facultative outcrossing (Glémin and Galtier, 2012); and 2) relative rates of ascospore production among species linked to potential differences in thallism could influence patterns of dominance in the BBD pathosystem and/or the perception of dominance where species-level determination are primarily made using ascospores from fieldcollected perithecia. In addition, MAT genes have been demonstrated as highly useful for determining phylogenetic relationships among species within a genus or clade (Lopes et al., 2018; O’Donnell et al., 2004; Turgeon, 1998).

Characterizing thallism can inform our understanding of disease cycle dynamics including propagule dissemination and mode of infection. One study investigating the production and dissemination of spores by $N$. ditissima infecting yellow birch (Betula alleghaniensis Britton) determined ascospores to be the dominant spore type in the environment throughout the year (Lortie and Kuntz, 1963). Additionally, ascospores of a related species Corinectria fuckeliana (C. Booth) C. González \& P. Chaverri (formerly Neonectria fuckeliana) have also been shown to be dominant relative to asexual conidia 
when infecting Pinus radiata D. Don in New Zealand (Crane et al., 2009). Together, these results indicate the progression of diseases caused by Neonectria and Corinectria fungi may depend on ascospore production and dissemination given the proposed limited dissemination of asexual conidia by these fungi (Lortie and Kuntz, 1963; Crane et al., 2009).

In addition to the potential importance of mating strategy on disease epidemiology, fungal population density assessments that depend on fruiting structure detection can be influenced by the relative rates of reproduction. Rates of visual detection are also likely influenced by fruiting structure type, where bright red perithecia are far easier to see and positively identify than the small, whitish conidiophores and sporodochia. Nearly all BBD studies that investigated interactions among these two Neonectria fungi have depended on the sampling and processing of perithecia to measure ascospores directly or culture the associated fungi for identification (Houston, 1994; Kasson and Livingston, 2009). These studies have indicated N. faginata, over time, supplants $N$. ditissima as the dominant pathogen in the BBD pathosystem. As with differences in optimal abiotic conditions (temperature, relative humidity) or seasonality of fruiting, differences in thallism - if they exist - could likewise influence the frequency of perithecia production and therefore, detection rates. Thus, determining thallism for $N$. ditissima, $N$. faginata and $N$. coccinea could enhance our ability to interpret patterns of relative abundance in the BBD system.

The objectives of this study were as follows: 1) Determine thallism among members of Neonectria with emphasis on BBD-associated fungi: N. ditissima, $N$. faginata, and $N$. coccinea. This is important as studies to determine the mating 
strategies of Neonectria fungi are limited. Furthermore, genomic data for many Neonectria spp. are lacking, and therefore, more general primers would be useful to permit broader characterization of mating type genes across species. 2) Confirm existing intra- and interspecies mating barriers using in vitro pairing assays. This is important as $N$. ditissima is reported from many plant hosts and the compatibility of strains from these various hosts remains unclear. Together, these results offer insight into mating regulation of Neonectria and allied fungi, thus providing an enhanced understanding of gene flow within and outside of the BBD system.

\section{Materials and Methods}

\section{Genome sequencing and identification of MAT loci}

Two genomes for $N$. faginata isolates (SK113 and MES1_34.1.1) were produced in association with this work and allowed for MAT gene discovery (Table 1). Full genome details will be provided in a forthcoming publication (Morrison and Garnas unpublished). Genomic data for the two $N$. faginata isolates of putatively opposite mating types as determined using the in vitro pairing assay described below were generated by a combination of Oxford Nanopore Technologies (ONT) and Illumina HiSeq sequencing. Genomic DNA was extracted from a N. faginata MAT1-1 isolate using a CTAB-chloroform DNA extraction method (van Diepen et al., 2017) and a MAT1-2 isolate using a Wizard ${ }^{\circledR}$ kit (Promega, Madison, WI, USA) and suspended in 75 $\mu$ l Tris-EDTA (TE) buffer (Amresco, Solon, OH, USA) with RNAse treatment to remove co-extracted RNA. The MAT1-1 isolate was sequenced using a MinION sequencer (Oxford Nanopore Technologies MIN-101B) using the unmodified 1D factory protocol (SQK-LSK109 protocol version DE_9062_v109_revD_23May2018) and a MIN-106 flow 
cell (FLOW MIN-106 R9 version). Both MAT1-1 and MAT1-2 isolates were subjected to Illumina HiSeq 2 x 250 PE sequencing at the University of New Hampshire Hubbard Center for Genome Studies. The ONT signal-level data was translated to FASTQ files using the Albacore v. 2.3.4 ONT proprietary basecaller resulting in $>960,000$ reads of which $50 \%$ were greater than $1 \mathrm{~Kb}$. ONT reads were quality controlled and assembled using the Canu assembler v. 1.8 (Koren et al., 2017) with initial genome size estimate of $45 \mathrm{Mb}$. Signal level ONT data was used to polish the assembly to correct major assembly errors using Nanopolish v. 0.10 .2 (Loman et al., 2015). Illumina HiSeq data was trimmed for adapter sequences and quality filtered using BBDuk (BBMap v. 38.58; Bushnell B, sourceforge.net/projects/bbmap/) resulting in 2.5 million and 11.8 million high-quality paired-end sequence reads for the MAT1-1 and MAT1-2 isolates, respectively. The MAT1-1 isolate Illumina sequences were subsequently used to further polish the Canu assembly using Pilon v. 1.22 (Walker et al., 2014). The MAT1-2 isolate sequence reads were assembled using SPAdes 3.13.1 with default settings. The resulting assemblies were assessed for contiguity using QUAST (Gurevich et al., 2013) and were checked for universal single-copy ortholog content using BUSCO v. 3.0.0 (Simão et al., 2015) with lineage Sordariomycetes. Assembly summary statistics are presented in Table S1, and complete code is available on request. Draft genome sequences are available on request.

Additionally, draft genomes of one European N. ditissima isolate (GenBank accession: LKCW01000000) (Gómez-Cortecero et al., 2015) and two New Zealand N. ditissima isolates (GenBank accessions: LDPK00000000; LDPL01000000) (Deng et al., 2015) were used to identify putative $N$. ditissima MAT idiomorphs. An unpublished $N$. 
coccinea draft genome (GenBank accession WPDF00000000, Castlebury et al., unpublished) also was used in this study.

To locate MAT loci within these genomes, we used NCBI GenBank tBLASTn algorithms with predicted MAT amino acid sequences derived from available MAT1-1 and MAT1-2 nucleotide sequences of two Fusarium (Nectriaceae) NCBI accessions: Fusarium anguioides (MH742713) and Fusarium tucumaniae (KF706656), respectively. Contigs containing sequences with an arbitrarily chosen similarity cutoff equal to or greater than $50 \%$ were selected for further examination.

\section{Characterizing the structure of MAT loci}

Genomic data were used to create genetic maps of the MAT1-1 and MAT1-2 loci for Neonectria ditissima and N. faginata as well as the MAT1-2 locus for N. coccinea. AUGUSTUS 3.3.1 (Stanke et al., 2008) was used to predict potential gene coding regions and their resulting amino acid sequences within putative MAT loci as well as to search for conserved genes within flanking regions up to 15,000 bp upstream and downstream of the MAT idiomorphs. The selected reference genome for this prediction was Fusarium graminearum, which is embedded in the AUGUSTUS software. NCBI GenBank BLASTp search algorithms were used identify genes by comparing predicted amino acid sequences to the NCBI protein database.

\section{Species-specific MAT 1-1-1 and MAT 1-2-1 primer design and PCR}

\section{amplification}

Once we had identified the MAT locus in each genome, we designed forward and reverse MAT1-1-1 and MAT1-2-1 specific primers for rapid characterization of mating 
type for $N$. ditissima and $N$. faginata isolates (Table 2). Excluding the primer binding sites, amplicon lengths were $710 \mathrm{bp}$ (MAT1-1-1) and 591 bp (MAT1-2-1) for $N$. ditissima. Amplicon lengths for N. faginata were 527 bp (MAT1-1-1) and 612 bp (MAT12-1) (Table 2). Primers were manually designed in polymorphic regions distinct to each Neonectria species with limited repeats and approximately $60 \%$ G/C content. Primer dimer and hairpin formation among primer pairs was assessed using AutoDimer (wwws.nist.gov/dnaAnalysis; Vallone and Butler, 2004). Melting temperatures were calculated using OligoAnalyzer Tool (Intergrated DNA Technologies, Coralville, IA, USA) for standard Taq polymerase. PCR products were generated in $25 \mu \mathrm{l}$ reactions containing $12.5 \mu$ Bioline PCR Master Mix (Bioline USA Inc, Taunton, MA), $10.0 \mu \mathrm{l} \mathrm{MG} \mathrm{H}_{2} \mathrm{O}, 1.5 \mu \mathrm{l}$ purified DNA, and $1.0 \mu \mathrm{l}$ of both MAT1-1 or MAT1-2 primers $(25 \mathrm{nM}$; Integrated DNA Technologies, Coralville, IA, USA). PCR conditions are outlined in Table 2 for each primer set. 
Table 1: Identity, source location, and host substrate of fungal isolates used in this study. GenBank accession numbers for derived and submitted sequences are included.

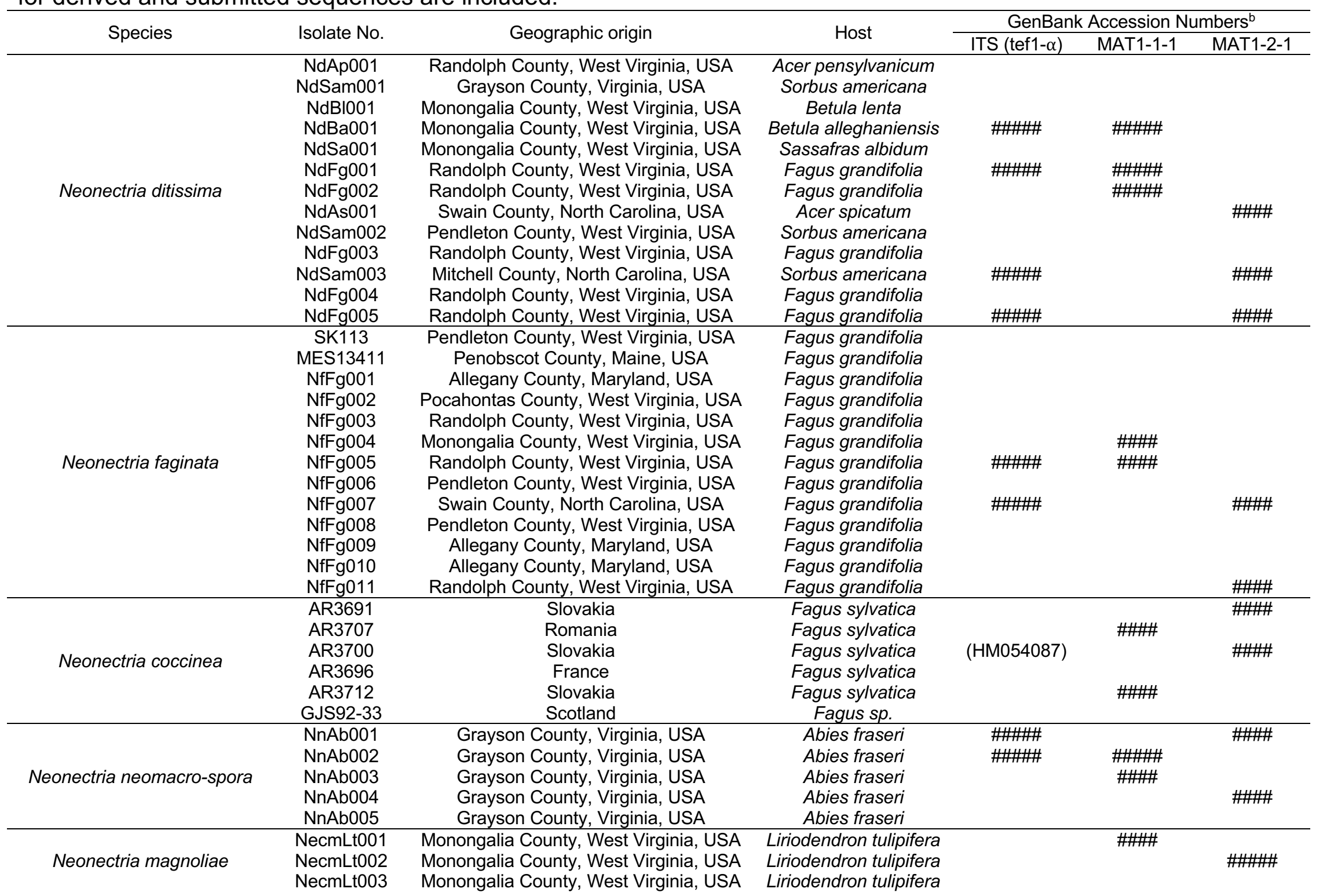




\begin{tabular}{|c|c|c|c|c|c|c|}
\hline & $\begin{array}{l}\text { NecmLt004 } \\
\text { NecmLt005 } \\
\text { NecmMf001 }\end{array}$ & $\begin{array}{l}\text { Tucker County, West Virginia, USA } \\
\text { Tucker County, West Virginia, USA } \\
\text { Randolph County, West Virginia, USA }\end{array}$ & $\begin{array}{l}\text { Liriodendron tulipifera } \\
\text { Liriodendron tulipifera } \\
\quad \text { Magnolia fraseri }\end{array}$ & \#\#\#\# & \#\#\#\# & \#\#\# \\
\hline Neonectria hederae & CBS714.97 & Netherlands & Hedera helix & & & \\
\hline Neonectria punicea & CBS119724 & Austria & Frangula alnus & & & \\
\hline Corinectria fuckeliana & IMI342667 & Switzerland & Picea sp. & & & \\
\hline Corinectria aff. fuckeliana & $\begin{array}{l}\text { CafPr001 } \\
\text { CafPr002 } \\
\text { CafPr003 } \\
\text { CafPr004 } \\
\text { CafPr005 } \\
\text { CafPr006 } \\
\end{array}$ & $\begin{array}{c}\text { Randolph County, West Virginia, USA } \\
\text { Grayson County, Virginia, USA } \\
\text { Grayson County, Virginia, USA } \\
\text { Randolph County, West Virginia, USA } \\
\text { Grayson County, Virginia, USA } \\
\text { Pocahontas County, West Virginia, USA } \\
\end{array}$ & $\begin{array}{l}\text { Picea rubens } \\
\text { Picea rubens } \\
\text { Picea rubens } \\
\text { Picea rubens } \\
\text { Picea rubens } \\
\text { Picea rubens } \\
\end{array}$ & \#\#\#\# & \#\#\# & $\begin{array}{l}\text { \#\#\#\# } \\
\# \# \#\end{array}$ \\
\hline Fusarium babinda & FbFg001 & Monongalia County, West Virginia, USA & Fagus grandifolia & $(\# \# \#)$ & & \#\#\#\# \\
\hline Gibberella fujikuroi & $\begin{array}{c}\mathrm{A} 00149 \\
\mathrm{G}-2\end{array}$ & & & & AF100925 & AB005041 \\
\hline Fusarium oxysporum & $\begin{array}{l}\mathrm{F} 23 \\
\mathrm{~F} 48 \\
\end{array}$ & & & & EU841022 & EU841047 \\
\hline Ustilaginoidea virens & $\begin{array}{l}\text { UVas2-26 } \\
\text { UVas2-23 } \\
\end{array}$ & & & & KM096573 & KM096575 \\
\hline Ophiocordyceps xuefengensis & $\begin{array}{l}\text { MHHNU7968 } \\
\text { MHHNU7966 }\end{array}$ & $\begin{array}{l}\text { Hunan Province, China } \\
\text { Hunan Province, China }\end{array}$ & & & $\mathrm{MH1}$ & $\begin{array}{l}76304 \\
\text { MH176301 }\end{array}$ \\
\hline
\end{tabular}


Table 2: MAT1-1-1 and MAT1-2-1 primers designed in this study, including the corresponding amplicon sizes and PCR protocols.

\begin{tabular}{|c|c|c|c|c|c|c|c|c|c|c|c|c|c|c|c|}
\hline Species & Target & Primer & $\mathrm{bp}$ & Primer Sequence (5' - 3') & $\begin{array}{l}\text { Cyc } \\
\text { les }\end{array}$ & $\begin{array}{r}\text { In } \\
\text { Dena }\end{array}$ & $\begin{array}{l}\text { ial } \\
\text { Iration }\end{array}$ & Dena & ration & Ann & aling & Ext & Ision & $\begin{aligned} F \\
\text { Ext }\end{aligned}$ & $\begin{array}{l}\text { al } \\
\text { sion }\end{array}$ \\
\hline \multirow[t]{2}{*}{ N. ditissima } & \multirow{2}{*}{ MAT1 } & NdM1f & \multirow{2}{*}{710} & TCAAGCTGTTCCCAGACG & \multirow{2}{*}{35} & \multirow{2}{*}{$95 \mathrm{C}$} & \multirow{2}{*}{$2: 00$} & \multirow{2}{*}{$95 \mathrm{C}$} & \multirow{2}{*}{$0: 30$} & \multirow{2}{*}{$53 \mathrm{C}$} & \multirow{2}{*}{$0: 30$} & \multirow{2}{*}{$72 \mathrm{C}$} & \multirow{2}{*}{$1: 00$} & \multirow{2}{*}{$72 \mathrm{C}$} & \multirow{2}{*}{ 7:00 } \\
\hline & & NdM1r & & GGCTTCGAACTCGTTGAG & & & & & & & & & & & \\
\hline \multirow{2}{*}{ N. ditissima } & \multirow{2}{*}{ MAT2 } & NdM2f & \multirow{2}{*}{590} & TCATGCCTTCTGAAGCC & \multirow{2}{*}{35} & \multirow{2}{*}{$95 \mathrm{C}$} & \multirow{2}{*}{$2: 00$} & \multirow{2}{*}{$95 \mathrm{C}$} & \multirow{2}{*}{$0: 30$} & \multirow{2}{*}{$52 \mathrm{C}$} & \multirow{2}{*}{$0: 30$} & \multirow{2}{*}{$72 \mathrm{C}$} & \multirow{2}{*}{ 1:00 } & \multirow{2}{*}{$72 \mathrm{C}$} & \multirow{2}{*}{ 7:00 } \\
\hline & & $\mathrm{NdM} 2 \mathrm{r}$ & & AAGCGCGACCGAGAATT & & & & & & & & & & & \\
\hline \multirow{2}{*}{ N. faginata } & \multirow{2}{*}{ MAT1 } & NfM1f & \multirow{2}{*}{530} & GCTAAGCGGCCTCTCAACG & \multirow{2}{*}{35} & \multirow{2}{*}{$95 \mathrm{C}$} & \multirow{2}{*}{$2: 00$} & & & & & & & & \\
\hline & & NfM1r & & GAGATACCGTACGTAGCCAGC & & & & 950 & 0.00 & 34 & 0.00 & 120 & 1.00 & 120 & 1.00 \\
\hline N. faginata & 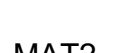 & NeoM2f & 610 & GCATGGACAAAGGCGTCG & 25 & 050 & חמח. & 050 & & 570 & & תמד & 1.00 & חיד & 7.00 \\
\hline & IVIATL & $\mathrm{NfM} 2 \mathrm{r}$ & 010 & GGAGCGGATGAAGGCCA & 35 & 950 & $2: 00$ & 950 & 0.30 & $5 / 0$ & 0.30 & $1 \angle C$ & T:U0 & $1 \angle \mathrm{C}$ & 1:00 \\
\hline N. ditissima / & & NeoM1f & & CGAGGTnGCTGCCAnGT & & & & & & & & & & & \\
\hline N.faginata & MAT1 & NeoM1r & 800 & AATGGGTTGTCGATGTCAATGG & 35 & $95 \mathrm{C}$ & 2:00 & $95 \mathrm{C}$ & $0: 30$ & $57 \mathrm{C}$ & 0:30 & $72 C$ & 1:00 & $72 \mathrm{C}$ & 7:00 \\
\hline N. ditissima / & & NeoM2f & & GCATGGACAAAGGCGTCG & & & & & & & & & & & \\
\hline N.faginata & MAT2 & NeoM2r & 610 & TCTGGGAnCGGATnAAGG & 35 & $95 \mathrm{C}$ & $2: 00$ & $95 \mathrm{C}$ & $0: 30$ & $57 \mathrm{C}$ & $0: 30$ & $72 \mathrm{C}$ & $1: 00$ & $72 \mathrm{C}$ & $7: 00$ \\
\hline Neonectria & & NeoM2Df & & $\begin{array}{c}\text { CGAGGKCGRTACTKGTAGTCRG } \\
\text { G }\end{array}$ & & & & & & & & & & & \\
\hline spp. & MAT2 & NeoM2Dr & 300 & $\begin{array}{c}\text { CCMAAYGCSTACATYCTKTACC } \\
\text { GYAA }\end{array}$ & 35 & $95 \mathrm{C}$ & 2:00 & $95 \mathrm{C}$ & $0: 30$ & $57 \mathrm{C}$ & 0:30 & $72 \mathrm{C}$ & $0: 45$ & $72 \mathrm{C}$ & 7:00 \\
\hline
\end{tabular}




\section{Genus-level MAT 1-1-1 and MAT 1-2-1 primer design and PCR amplification}

Genus-level primer pairs intended to amplify MAT1-1-1 (NeoM1f and NeoM1r) and MAT1-2-1 (NeoM2f and NeoM2r) for all included Neonectria species were designed as described above using available Neonectria genomic data (Table 2). Primer development for Neonectria spp. was completed using MAT1-1-1 and MAT1-2-1 sequences derived from N. ditissima, N. faginata, N. coccinea (MAT1-2-1), N. punicea ([J.C. Schmidt] Castl. \& Rossman) (MAT1-1-1; GenBank accession:

QGQA00000000.1), and N. hederae genomes ([C. Booth] Castl. \& Rossman) (MAT1-21; GenBank accession: QGQB00000000.1). MAT1-1-1 and MAT1-2-1 nucleotide sequences were separately aligned using CLUSTAL-W (Larkin et al., 2007) within MEGA v10.1.7 (Stecher et al., 2018), and primers were designed within conserved regions to potentially increase the utility of these primers for other Neonectria and allied fungi. MAT1-2-1 exhibited a higher level of polymorphism among the included Neonectria species and therefore, a second set of degenerate primers were designed to amplify the MAT1-2-1 gene (Table 2; NeoM2Df and NeoM2Dr). All the sequences generated were deposited in GenBank (Table 1).

\section{Selection of Isolates}

Single ascospore-derived isolates of $N$. ditissima and $N$. faginata recovered from various geographic locations and host substrates (Table 1) were generated by squashing a single perithecium in $1 \mathrm{ml}$ of sterile water within a $1.5 \mathrm{ml}$ Eppendorf tube, vortexing for 15 seconds to disperse ascospores, and spreading $100 \mu$ l onto a glucoseyeast extract (GYE) medium. Five germinating ascospores were transferred to a new GYE plate using a sterile needle after 24 hours and were allowed to grow for one week 
before replating. Each isolate was grown on GYE for two weeks and then identified using colony morphology based on type descriptions (Castlebury et al., 2006).

Genomic DNA was extracted from isolates or directly from perithecia as described above. For each species identified morphologically, three random isolate identifications were confirmed by sequencing the ribosomal internal transcribed spacer region (ITS) using primers ITS5 (5' - GGAAGTAAAAGTCGTAACAAGG - 3') and ITS4 (5' - TCCTCCGCTTATTGATATGC - 3') (White et al., 1990). The PCR protocol was as follows: $95^{\circ} \mathrm{C}$ for 2 min followed by 35 cycles of $95^{\circ} \mathrm{C}$ for $30 \mathrm{sec}, 56^{\circ} \mathrm{C}$ for $30 \mathrm{sec}, 72$ ${ }^{\circ} \mathrm{C}$ for $1 \mathrm{~min}$ with a final extension at $72{ }^{\circ} \mathrm{C}$ for $7 \mathrm{~min}$. EF1- $\alpha$ sequencing was completed using primers EF1728F (5' - CATCGAGAAGTTCGAGAAGG - 3') (Carbone and Kohn, 1999) and EF1-1567R (5' - ACHGTRCCRATACCACCRATCTT - 3') (Rehner 2001) with the following PCR protocol: $95^{\circ} \mathrm{C}$ for 2 min followed by 35 cycles of $95^{\circ} \mathrm{C}$ for 30 sec, $55^{\circ} \mathrm{C}$ for $1 \mathrm{~min}, 72^{\circ} \mathrm{C}$ for $1 \mathrm{~min}$ with a final extension at $72{ }^{\circ} \mathrm{C}$ for $7 \mathrm{~min}$.

PCR products were visualized with gel electrophoresis by adding $4 \mu$ I SYBR gold (Invitrogen, Grand Island, NY, USA) and $4 \mu$ loading dye (5Prime, Gaithersburg, MD) to products. Samples were then loaded into a 1.5\%, wt/vol, agarose gel (Amresco, Solon, $\mathrm{OH}, \mathrm{USA}$ ) made with $0.5 \%$ Tris-borate-EDTA buffer (Amresco, Solon, OH, USA). To compare sizes, 100-bp and 1-kb molecular ladders (Omega Bio-tek, Norcross, GA, USA) also were added to gels. Electrophoresis was performed at $90 \mathrm{~V}$ for 75 minutes. Bands were visualized on a UV transilluminator (Syngene, Frederick, MD, USA).

Positive reactions were prepared for sequencing using ExoSAP-IT (Affymetrix, Santa Clara, CA, USA) according to the manufacturer's recommendations. Purified PCR products were Sanger sequenced in forward and reverse directions using the 
same PCR primers (Eurofins, Huntsville, AL, USA). BLASTn searches were then used to identify species based on the best match in the NCBI database.

\section{Mating type gene screening}

All selected $N$. ditissima and $N$. faginata (22 and 18 isolates, respectively) were screened for the presence of MAT1-1-1 and/or MAT1-2-1 using species-specific and genus-level primer sets with the PCR protocols listed in Table 2. All PCR products were visualized, and a subset of positive reactions were sequenced as described for ITS and EF1- $\alpha$ amplicons.

To confirm the specificity of species-specific primer sets, mating type PCR reactions were performed using $N$. ditissima MAT primers for $N$. faginata isolates and vice versa. Additionally, a number of other members of the Nectriaceae also were tested using both $N$. ditissima and $N$. faginata MAT primers as described above. These included isolates of N. coccinea, N. neomacrospora ([C. Booth \& Samuels] Mantiri \& Samuels), Fusarium babinda (Summerell, C.A. Rugg \& L.W. Burgess, 1995), Nectria magnoliae (M.L. Lohman \& Hepting, 1943) and an additional unresolved species for which additional data is needed to confirm identity: Corinectria aff. fuckeliana $(99.37 \%$ EF1- $\alpha$ sequence similarity to NCBI Genbank accession MK911707.1) (Table 1). Genuslevel Neonectria MAT primer sets were similarly tested using representatives from all five Nectriaceae species tested in this study.

All resulting sequences were aligned as described above and compared to genome-derived MAT1-1-1 or MAT1-2-1 sequences to confirm their identity based on a sequence similarity. All sequences having greater than $70 \%$ sequence similarity were selected for further analysis. This threshold was determined by comparing MAT1-1-1 
and MAT1-2-1 genome-derived sequences from N. ditissima to the more distantly related Fusarium anguioides (MAT1-1-1; Genbank Accession: MH742713) and Fusarium tucumaniae (MAT1-2-1; Genbank Accession: KF706656) sequences.

\section{Phylogenetic analysis and protein alignment}

To examine evolutionary patterns and divergence in mating type genes, we constructed phylogenetic trees using the mating type gene sequence data produced in this study together with comparable sequences for other Nectriaceae available in NCBI Genbank, including 11 strains representing 7 species (Table 1). All analyses were completed using MEGA v10.1.7 (Stecher et al., 2018). MAT nucleotide sequences were aligned using CLUSTAL-W (Larkin et al. 2007) and the best-fit nucleotide substitution model was chosen using Model Test AICc scores in MEGA v10.1.7. MAT1-1-1 and MAT1-2-1 maximum-likelihood trees were constructed independently using the Kimura 2-parameter model with gamma distribution $(K 2+G)$ and 1000 bootstrap replicates. For both trees, MAT1-1-1 and MAT1-2-1 sequences from Ophiocordyceps xeufengensis and Ustilaginoidea virens served as outgroup taxa (Table 1).

We performed protein alignments to characterized divergence among species that could play a role in the maintenance of mating barriers. Protein sequences were predicted from one MAT1-1-1 and one MAT1-2-1 coding sequence from each species using ExPASy-Translate tool (https://web.expasy.org/translate/). Resulting protein sequences were aligned using CLUSTAL-W within MEGA v10.1.7. Boxshade Server v.3.21 (https://embnet.vital-it.ch/software/BOX_form.html) was used to visualize shared amino acids within each sequence. 


\section{In vitro mating assay}

To demonstrate mating among MAT1-1 and MAT1-2 strains, an in vitro mating assay was performed with six MAT1-1-1 and six MAT1-2-1 isolates of both N. faginata and $N$. ditissima. These isolates varied in geographic origin and for $N$. ditissima, host substrate as to test compatibility among representatives from allopatric populations and potentially host-specific $N$. ditissima isolates. This pairing assay did not include $N$. coccinea as only genomic DNA was available. Selected isolates were grown on glucose-yeast extract agar for two weeks. Each selected isolate was then paired three times with itself, with an isolate of the same mating type, and with two isolates of the opposite mating type for a total of twelve pairings per isolate. For each pairing, a 5x5$\mathrm{mm}$ fungus-colonized agar plug from each isolate was placed on opposite sides of a sterile, flat toothpick placed atop GYE media along the center point of the petri dish (Figure $1 \mathrm{~A}$ ). All plates were parafilmed and placed at $20^{\circ} \mathrm{C}$ with a $16 \mathrm{~h} / 8 \mathrm{~h}$ light/dark cycle under cool fluorescent lamps. Plates were checked weekly for perithecia formation for up to 12 weeks (Figure 1B). 


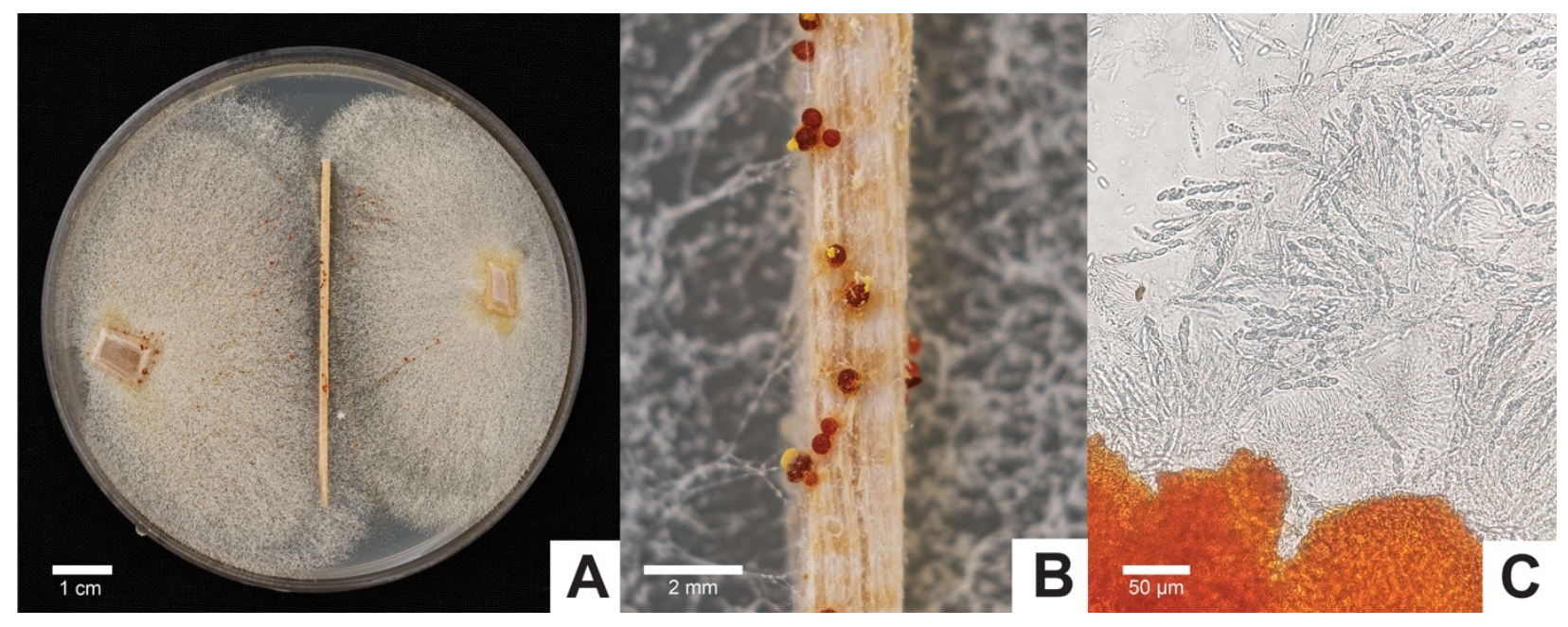

Figure 1: In vitro mating assay for Neonectria spp. and related fungi: A) Approximately $5 \mathrm{~mm} \times 5 \mathrm{~mm}$ colonized plugs of confirmed MAT1-1 and/or MAT1-2 strains placed on either side of a sterile wooden toothpick placed upon GYE media; B) Perithecia exuding ascospores on toothpick after approximately 12 weeks; C) Perithecia squash mount showing ascospores within asci. Neonectria magnoliae shown in figure. Individual scale bars are included in each panel.

As perithecia were produced, squash mounts were used to check for ascospores using light microscopy (Figure 1C). Additionally, ascospores were checked for viability by removing a single perithecium, macerating in $1 \mathrm{ml}$ of sterile $\mathrm{dH}_{2} \mathrm{O}$, spreading $300 \mu \mathrm{l}$ of the spore suspension on GYE, and observing growth. For one progeny plate from each paired isolate set, ten germinating ascospores were sub-cultured onto a new GYE plate after 24 hours and allowed to grow for one week. Genomic DNA was extracted from the ten subcultured progeny and screened for MAT genes as described above. Given that progeny should segregate 1:1 for MAT1-1 and MAT1-2, all ascospore suspension plates were incubated for up to 12 weeks to observe mating among the progeny. 
A subsequent interspecies mating assay was performed to test mating capability among $N$. ditissima, $N$. faginata, and other Nectriaceous fungi. Here, MAT1-1- and MAT1-2-positive isolates of N. ditissima (MAT1-1: NdBI001; MAT1-2: NdSam003) and N. faginata (MAT1-1: NfFg005; MAT1-2: NfFg008) were paired three times each with an isolate of Nectria magnoliae (isolate no. NecmLt005) and Corinectria aff. fuckeliana (isolate no. CafPr004) as described above. Additionally, both N. ditissima isolates were paired three times with the $N$. faginata isolate of the opposite mating type. Plates were checked weekly for perithecia formation for up to 12 weeks and processed as described above.

\section{Results}

\section{Identification and structure of MAT loci in N. ditissima and $\mathbf{N}$. faginata}

Each of the $N$. faginata and $N$. ditissima genomes contained either the MAT1-1 or MAT 1-2 idiomorph, and the single N. coccinea genome contained only a MAT1-2 idiomorph. MAT genes of the opposite mating type were not found within the genomes. AUGUSTUS analyses of the MAT loci and flanking genes revealed a similar genetic structure for $N$. coccinea, $N$. ditissima and $N$. faginata with only minor differences in the MAT gene open reading frame (ORF) and intron lengths (Figure 2). The MAT1-1-1 coding sequence for $N$. ditissima was 1,167 bp (357 amino acids [aa]; GenBank accession XXXXX) with two introns of $48 \mathrm{bp}$ and $46 \mathrm{bp}$, while the MAT1-1-1 coding sequence for N. faginata was 1204 bp (371 aa; GenBank accession XXXXX) with two introns of $46 \mathrm{bp}$ and $44 \mathrm{bp}$. The MAT1-2-1 coding sequence for N. ditissima was $827 \mathrm{bp}$ (243 aa; GenBank accession XXXXX) with two introns of 48 bp and $49 \mathrm{bp}$, while the coding sequence for $N$. faginata was 815 bp (239 aa; GenBank accession XXXXX) with 
two introns of $47 \mathrm{bp}$ and $50 \mathrm{bp}$. Additionally, the genetic structure of MAT1-2-1 of $N$. coccinea was found to be similar to $N$. ditissima and $N$. faginata with an coding sequence of 816 (238 aa; GenBank accession XXXXX) with two introns of 50 bp and 48 bp.

Two commonly co-occurring MAT associated genes were found near MAT1-1-1 in both $N$. ditissima and $N$. faginata, including MAT1-1-2 and MAT1-1-3 (Figure 2; Coppin et al., 1997). For each of the three Neonectria spp., evidence of co-occurrence of MAT1-2-1 and MAT1-2-2 was not found. Both N. ditissima and N. faginata MAT loci were flanked by the conserved SLA2 gene previously described as being associated with MAT loci (Debuchy and Turgeon, 2006). SLA2 was found to occur in a separate contig of the $N$. coccinea de novo genome assembly, and although present, could not be shown to be part of the MAT locus without further assembly. APN2 is a second conserved gene often flanking the MAT loci of other Ascomycetes (Debuchy and Turgeon, 2006), but this gene was not identified in the regions flanking the MAT loci using the de novo genome assemblies analyzed in this study. 
N. ditissima MAT1-1 Idiomorph
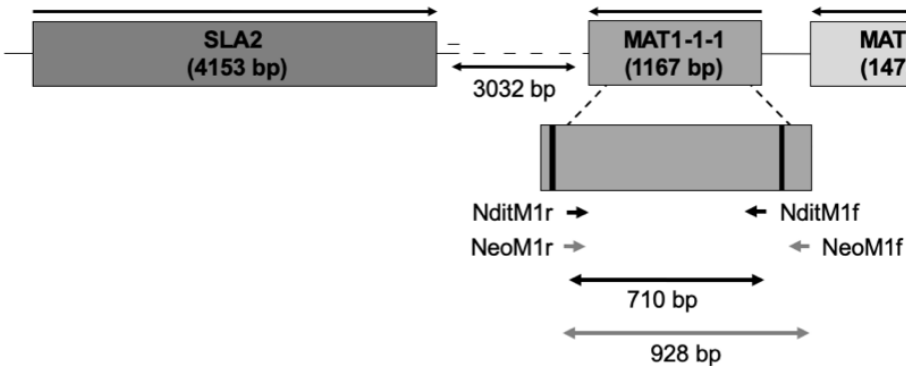

N. ditissima MAT1-2 Idiomorph
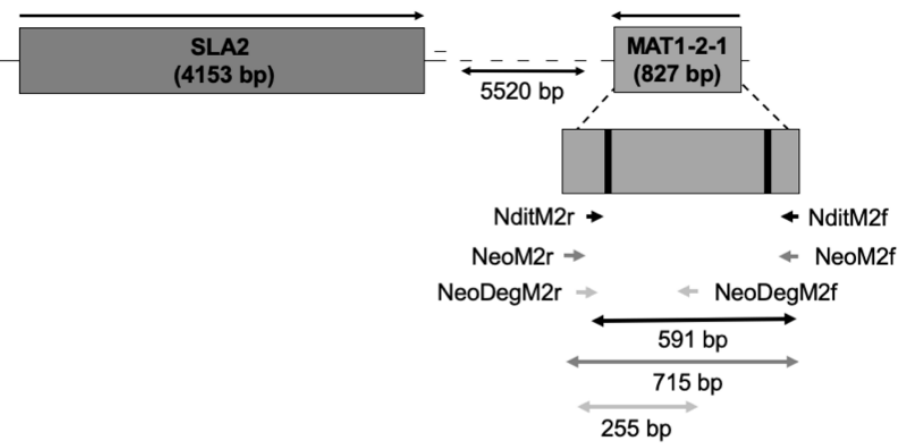

N. faginata MAT1-1 Idiomorph

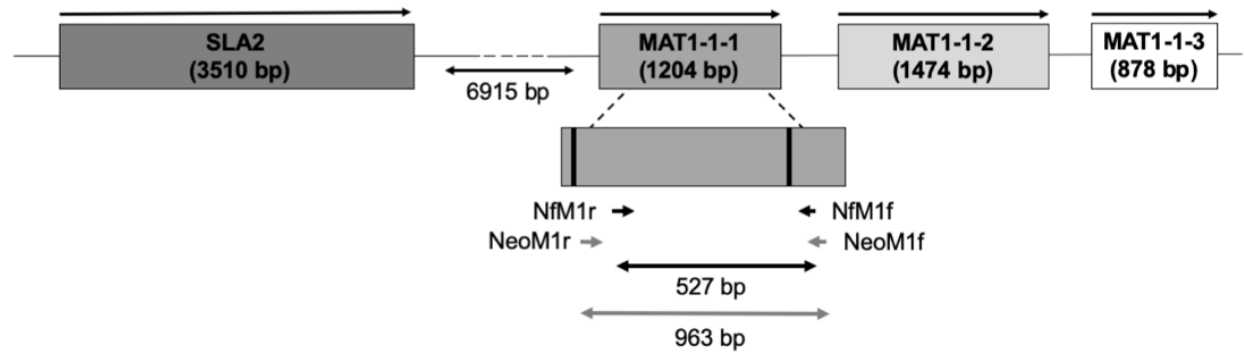

N. faginata MAT1-2 Idiomorph

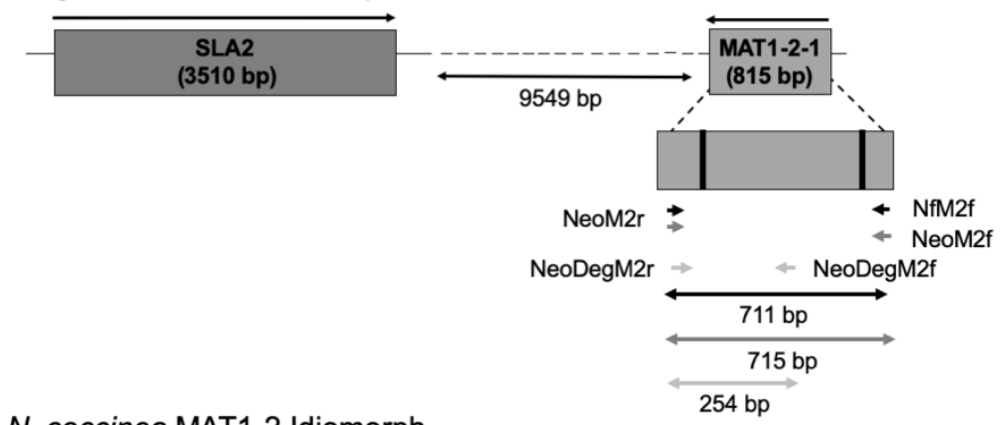

N. coccinea MAT1-2 Idiomorph

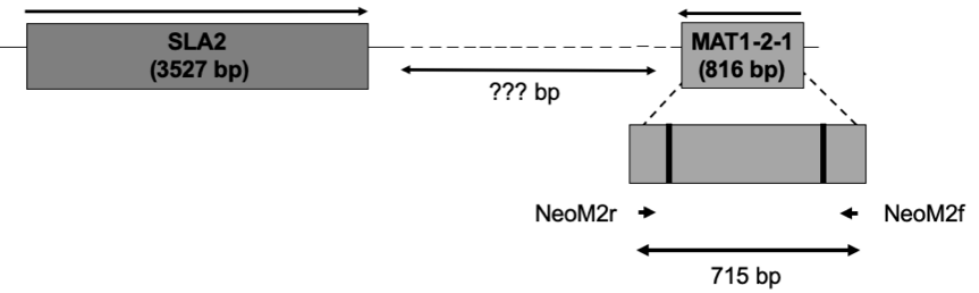

$\overline{0.5 \mathrm{~kb}}$ 
Figure 2: Structure of the MAT1-1 and MAT1-2 loci of the heterothallic fungi: Neonectria ditissima, Neonectria faginata, and N. coccinea (MAT1-2 only). Arrows above the genes indicate the $5^{\prime}-3^{\prime}$ orientation. Coding sequence lengths are included below each gene identifier. For MAT1-1-1 and MAT1-2-1 genes, introns are represented by vertical black lines, and approximate primer binding locations are illustrated for each primer pair below the gene illustration. The approximate amplicon size for each primer pair is shown below the primer binding locations and is shaded based on each primer pair. All distances and sizes are estimations and not drawn to scale.

\section{Species specific MAT1-1-1 and MAT1-2-1 primers}

Neonectria ditissima (NdM1f/r) and $N$. faginata (NdM2f/r) MAT primer pairs amplified a single product of the expected size for their target species (Table 2). Sequencing confirmed the identity of all PCR products, each of which exhibited $99 \%$ or greater sequence identity with the target MAT sequences. All of the 160 (40 initially screened or 120 progenies from in vitro crosses) single-spore derived isolates tested yielded either MAT1-1-1 or MAT1-2-1 amplicons while DNA extractions from perithecia $(\mathrm{N}=7)$ containing ascospores of both mating types yielded both MAT products.

Species-specific primers designed for $N$. faginata did not amplify either MAT gene in $N$. ditissima. Likewise, $N$. ditissima-specific primers did not amplify either MAT gene in $N$. faginata.

MAT1-1-1 and MAT1-2-1 primer pairs designed for N. ditissima did not amplify DNA in any of the other tested species (Table 3). In contrast, the N. faginata MAT1-1-1 primer pair amplified MAT1-1-1 in MAT1-1 isolates in N. coccinea, N. neomacrospora, 
and Nectria magnoliae. In contrast, MAT1-2-1 primers for N. faginata did not amplify MAT1-2-1 for any other tested species.

Table 3: Summary PCR results for each included species and primer pair with ' + denoting successful amplification of the target sequence and "*" indicating non-target amplification when primer pair is applied to an isolate of the opposite mating type. Empty cells indicate no PCR product was amplified.

\begin{tabular}{|c|c|c|c|c|c|c|c|}
\hline \multirow{2}{*}{ Species } & \multicolumn{7}{|c|}{ Primer Set } \\
\hline & NdM1 & $\mathrm{NdM} 2$ & NfM1 & NfM2 & NeoM1 & NeoM2 & NeoDegM2 \\
\hline Neonectria ditissima & + & + & & & + & + & + \\
\hline Neonectria faginata & & & + & + & + & + & + \\
\hline Neonectria coccinea & & & $+*$ & * & & * & + \\
\hline $\begin{array}{c}\text { Neonectria } \\
\text { neomacrospora }\end{array}$ & & & + & & & * & + \\
\hline Nectria magnoliae & & & + & & & & + \\
\hline Corinectria aff. fuckeliana & & & & * & + & & + \\
\hline Fusarium concolor & & & & & & & + \\
\hline
\end{tabular}

${ }^{*}=$ non-target amplification when primer pair is applied to isolates containing the opposite MAT idiomorph

Amplification of non-mating type associated proteins was observed when applying N. faginata MAT1-1-1 primers to MAT1-2 strains of N. coccinea, Corinectria aff. fuckeliana, and N. neomacrospora. Sequencing of these amplicons revealed a putative major facilitator superfamily (MFS-type) transporter protein $(\sim 120 \mathrm{bp}>$ target) in $N$. coccinea and two undescribed hypothetical proteins in $N$. neomacrospora ( 470 bp $>$ target) and C. aff. fuckeliana ( 470 bp > target) (Table S2). Additionally, amplification of non-mating type associated proteins was observed when $N$. faginata MAT1-2-1 primers were applied to MAT1-1 N. coccinea and C. aff. fuckeliana isolates 
including hypothetical proteins in N. coccinea ( 540 bp > target) and C. aff. fuckeliana ( 190 bp > target) (Table S2).

\section{Genus-level Neonectria MAT1-1-1 and MAT1-2-1 primers}

Genus-level MAT primer pairs (NeoM1f/r and NeoM2f/r) successfully amplified both MAT1-1-1 and MAT1-2-1 gene for N. ditissima and N. faginata (Supplemental Figure 1). These same primers also amplified MAT1-1-1 for Corinectria aff. fuckeliana (Table 3) but failed to amplify MAT1-1-1 and MAT1-2-1 for all other non-target fungi.

The MAT1-2-1 degenerate primer pair (NeoM1df/r) successfully amplified MAT12-1 for all species tested, including a more distantly related Fusarium babinda isolate. Non-target amplification of a hypothetical protein ( $\sim 590 \mathrm{bp}>$ target) was observed when the NeoM2f/r primer pair was applied to MAT1-1 N. coccinea and both MAT1-1 and MAT1-2 N. neomacrospora isolates.

\section{Phylogenetic analyses and protein alignments}

Phylogenetic analyses of MAT1-1-1 and MAT1-2-1 sequences resulted in similar tree topologies (Figures 3 and 4). Analysis of MAT1-1-1 grouped all Neonectria species into a strongly supported monophyletic clade sister to Corinectria (containing several species formerly classified in Neonectria) (González and Chaverri, 2017) (Figure 3). Within Neonectria, a clade containing N. coccinea, N. faginata and N. punicea was resolved as sister to Nectria magnoliae from Liriodendron tulipifera and Magnolia fraseri. For MAT1-2-1, all included Neonectria species resolved to a monophyletic clade that was sister to a clade containing Corinectria aff. fuckeliana and the three species of Fusarium (Figure 4). Additionally, two sister clades within Neonectria were resolved: 1) 
$N$. faginata was sister to a clade containing N. coccinea and Nectria magnoliae, and 2) Neonectria ditissima and $N$. neomacrospora formed a monophyletic clade sister to $N$. hederae. All isolates of $N$. ditissima formed a single lineage regardless of plant host for both MAT1-1-1 and MAT1-2-1.

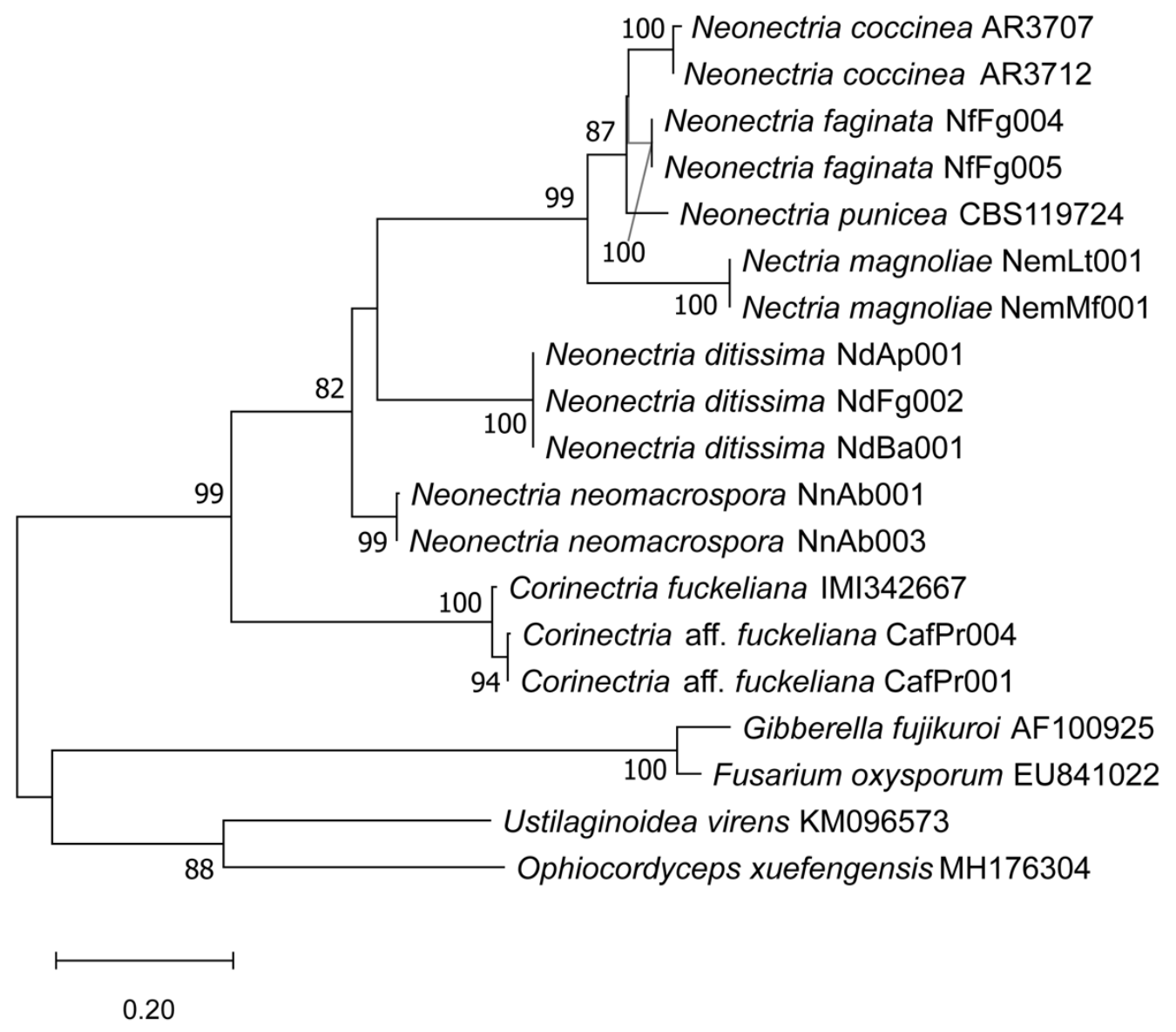

Figure 3: Phylogenetic relationships of Neonectria spp. and related fungi based on MAT1-1-1 gene sequence data. The phylogeny was inferred using a Maximum Likelihood analysis based on the Kimura 2-parameter model with gamma distribution $(K 2+G)$ and 1000 bootstrap replicates. Bootstrap values $>70 \%$ are given at the nodes. Branch lengths represent the number of substitutions per site. Outgroup includes Ophiocordyceps xeufengensis and Ustilaginoidea virens. 


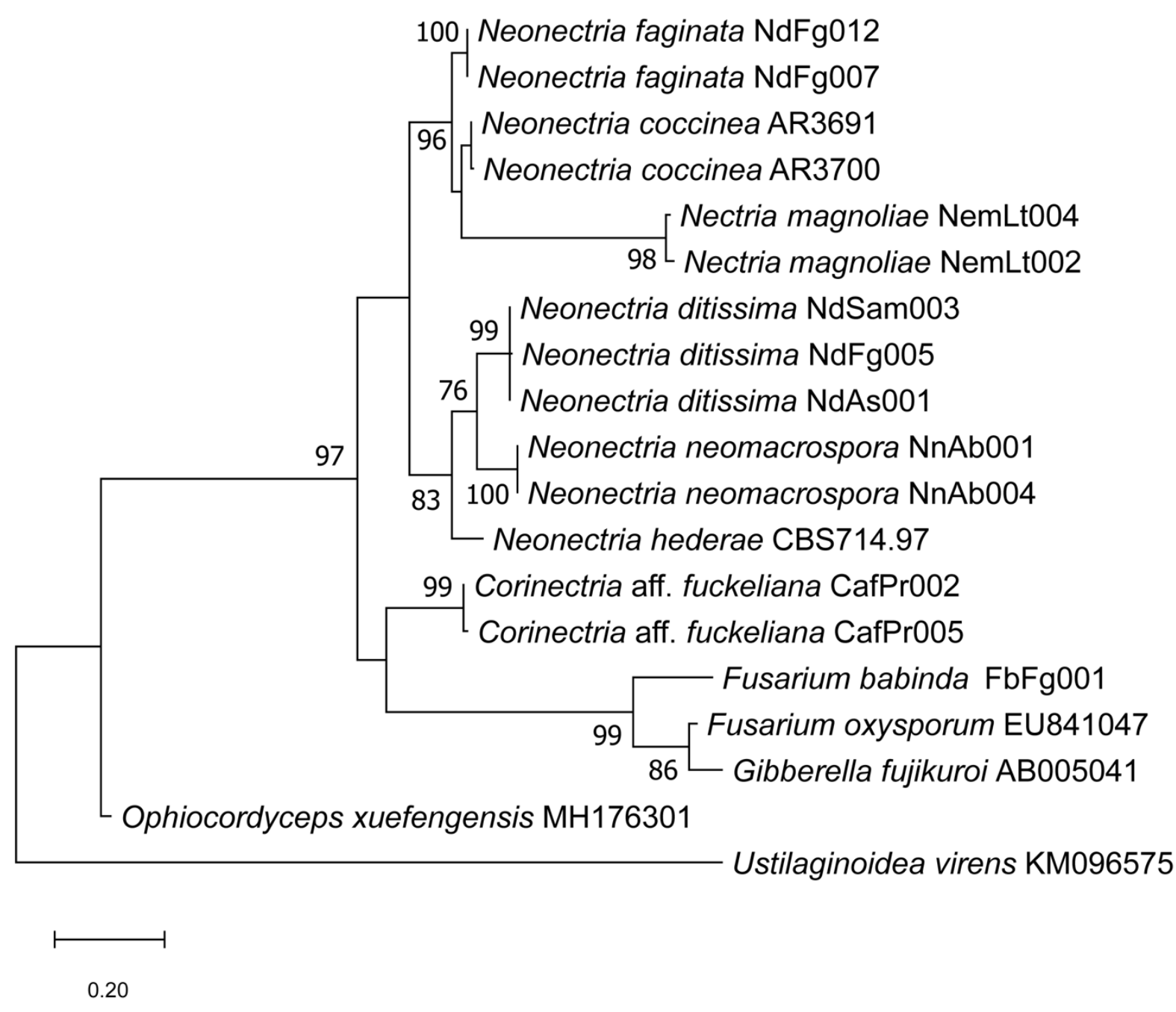

Figure 4: Phylogenetic relationships of Neonectria spp. and related fungi based on MAT1-2-1 gene sequence data. The phylogeny was inferred using a Maximum Likelihood analysis based on the Kimura 2-parameter model with gamma distribution $(\mathrm{K} 2+\mathrm{G})$ and 1000 bootstrap replicates. Bootstrap values $>70 \%$ are given at the nodes. Branch lengths represent the number of substitutions per site. Outgroup includes Ophiocordyceps xeufengensis and Ustilaginoidea virens. 
Protein alignments included approximately 100 amino acids for MAT1-1-1 sequences and approximately 75 amino acids for MAT1-2-1 sequences within the conserved region of these genes (Figure 5). The protein sequences deduced from Neonectria coccinea, Nectria magnoliae, Corinectria aff. fuckeliana, and Fusarium babinda MAT1-2-1 PCR products were missing the first five amino acids due to lacking primer binding locations, and therefore, results reported for MAT1-2-1 below represent values with (full) and without (partial) the first five amino acids.Protein alignments for both MAT1-1-1 and MAT1-2-1 were most similar among Neonectria spp. with $28 \%$ and $29.3 \%$ (or $36 \%$ for full sequence comparisons) of amino acids conserved across all species considered, respectively. Of these shared amino acids, 19\% MAT1-1-1 and 25.3\% MAT1-2-1 amino acids (partial seqs) were shared with included Corinectria spp. Across all included fungi, only $5 \%$ of amino acids were shared among MAT1-1-1 sequences and $21.3 \%$ (or $28 \%$ for full sequence comparisons) amino acids were shared among MAT1-2-1 sequences. 
MAT1-1-1

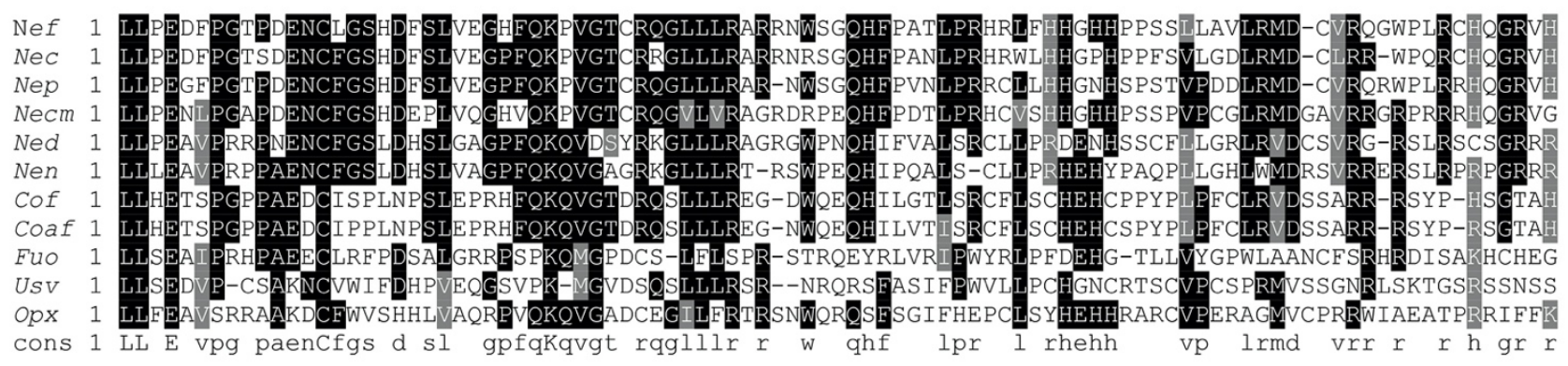

MAT1-2-1

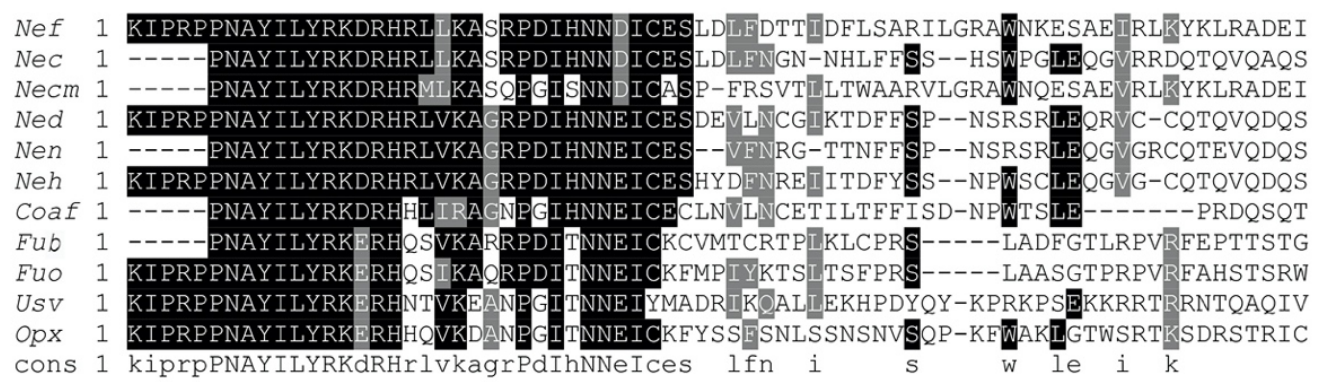

Figure 5: MAT1-1-1 and MAT1-2-1 amino acid alignments including a selection of study fungal species. Name abbreviations are as follows: Nef $=$ Neonectria faginata; $N e c=$ Neonectria coccinea; Nep $=$ Neonectria punicea; Necm = Nectria magnoliae; Ned = Neonectria ditissima; Nen = Neonectria neomacrospora; Neh = Neonectria hederae; Cof = Corinectria fuckeliana; Coaf = Corinectria aff. fuckeliana; Fub = Fusarium babinda; Fuo = Fusarium oxysporum; Usv = Ustilaginoidea virens; Opx = Ophiocordyceps xuefengensis; Cons = consensus. Uppercase letters within the consensus sequence represent positions with identical amino acids. Lower case letters represent positions with similar amino acids. Black shading represents conserved amino acids among $50 \%$ or more species. Grey shading represents shared amino acids with shared characteristics/properties among species. 


\section{In vitro pairing assay results}

For N. faginata and N. ditissima intraspecies pairings, perithecia with viable ascospores were produced for all pairings between MAT1-1 and MAT1-2 strains with one exception: one pairing between isolates $\mathrm{NfFg006}$ and $\mathrm{NfFg} 012$ failed to produce perithecia within the 12-week assay (Table 4). All ascospore suspension plates containing ascospore progeny from single, squashed perithecia resulting from MAT1-1 $x$ MAT1-2 pairings also yielded perithecia. No pairings with an isolate of the same mating type, including self-pairings, yielded perithecia. Additionally, no evidence of mating was observed in interspecies pairings.

Table 4: In vitro pairing assay results for $N$. ditissima and $N$. faginata. PCR confirmed MAT identities are included for each isolate. Three pairings were completed for each isolate set. Result format $=$ number of positive results out of the three total pairings. Results categories include the following: 1. Production of perithecia; 2 . Presence of ascospores within perithecia 3. Viability of ascospores from ascospore suspension plates; 4 . Production of perithecia on ascospore suspension plates used for viability confirmation.

\begin{tabular}{|c|c|c|c|c|c|c|c|}
\hline \multicolumn{2}{|c|}{ Isolate A } & \multicolumn{2}{|c|}{ Isolate B } & \multirow[t]{2}{*}{ Perthecia } & \multirow[t]{2}{*}{ Ascospores } & \multirow[t]{2}{*}{ Viability } & \multirow{2}{*}{$\begin{array}{c}\text { Progeny } \\
\text { Perithecia }\end{array}$} \\
\hline ID & MAT & ID & MAT & & & & \\
\hline NdAp001 & MAT1 & $\mathrm{NdAs} 001$ & MAT2 & 3 & 3 & 3 & 3 \\
\hline NdSam001 & MAT1 & NdSam002 & MAT2 & 3 & 3 & 3 & 3 \\
\hline NdBI001 & MAT1 & $\mathrm{NdFg003}$ & MAT2 & 3 & 3 & 3 & 3 \\
\hline $\mathrm{NdBa} 001$ & MAT1 & NdSam003 & MAT2 & 3 & 3 & 3 & 3 \\
\hline $\mathrm{NdSa001}$ & MAT1 & $\mathrm{NdFg004}$ & MAT2 & 3 & 3 & 3 & 3 \\
\hline NdFg002 & MAT1 & NdFg005 & MAT2 & 3 & 3 & 3 & 3 \\
\hline NdAp001 & MAT1 & NdSam001 & MAT1 & 0 & - & - & - \\
\hline NdBI001 & MAT1 & $\mathrm{NdBa001}$ & MAT1 & 0 & - & - & - \\
\hline NdSa001 & MAT1 & NdFg002 & MAT1 & 0 & - & - & - \\
\hline
\end{tabular}




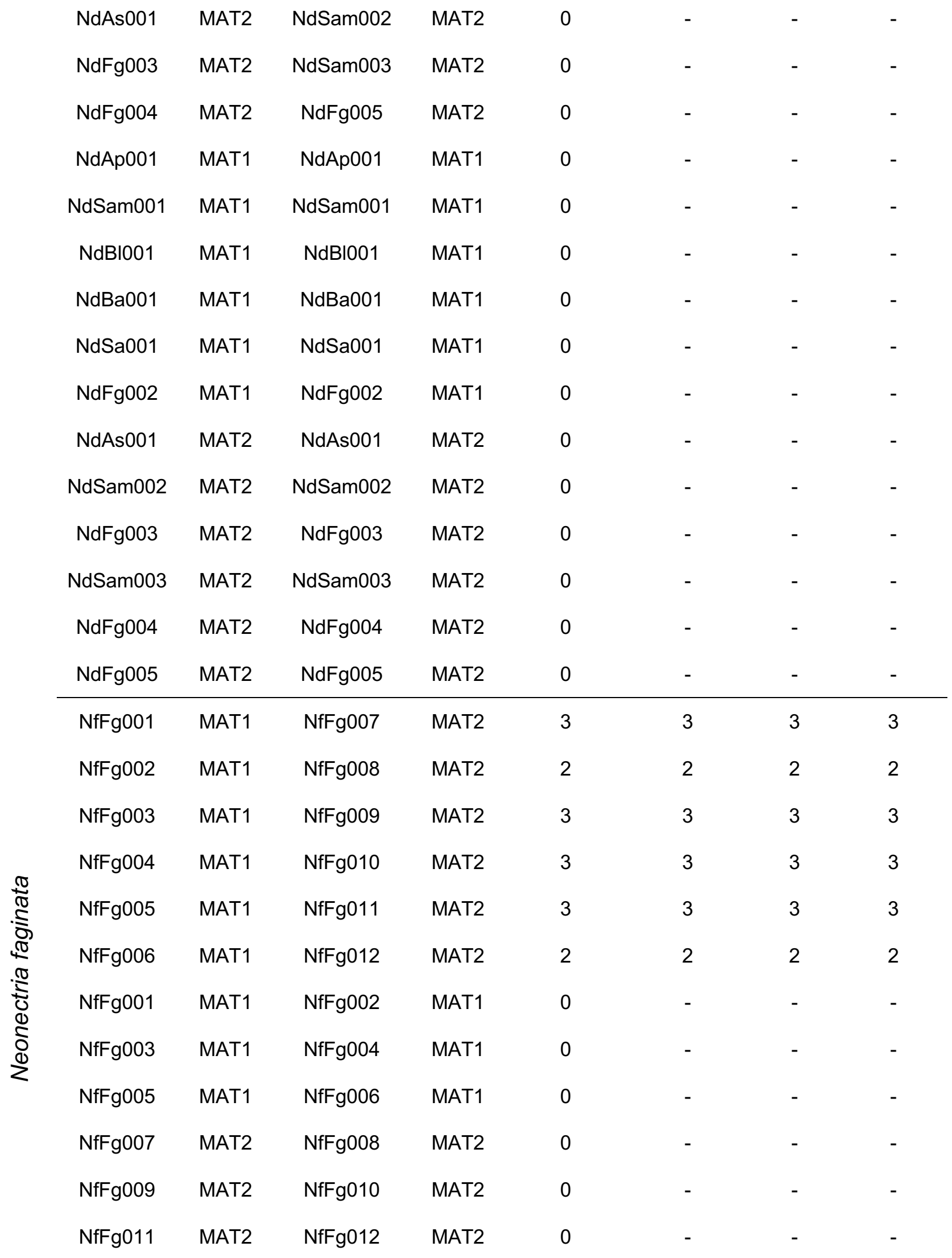




\begin{tabular}{lllllll} 
NfFg001 & MAT1 & NfFg001 & MAT1 & 0 & - & - \\
NfFg002 & MAT1 & NfFg002 & MAT1 & 0 & - & - \\
NfFg003 & MAT1 & NfFg003 & MAT1 & 0 & - & - \\
NfFg004 & MAT1 & NfFg004 & MAT1 & 0 & - & - \\
NfFg005 & MAT1 & NfFg005 & MAT1 & 0 & - & - \\
NfFg006 & MAT1 & NfFg006 & MAT1 & 0 & - & - \\
NfFg007 & MAT2 & NfFg007 & MAT2 & 0 & - & - \\
NfFg008 & MAT2 & NfFg008 & MAT2 & 0 & - & - \\
NfFg009 & MAT2 & NfFg009 & MAT2 & 0 & - & - \\
NfFg010 & MAT2 & NfFg010 & MAT2 & 0 & - & - \\
NfFg011 & MAT2 & NfFg011 & MAT2 & 0 & - & - \\
NfFg012 & MAT2 & NfFg012 & MAT2 & 0 & - & - \\
\hline
\end{tabular}

Each progeny included in the subsequent PCR screening resulted in the amplification of a single MAT gene. Both MAT genes were found to be segregated among progeny at a ratio not significantly divergent from the expected 1:1 for $N$. ditissima $\left(\chi^{2}=0.07, \mathrm{df}=1,60 ; P=0.80\right)$ and $N$. faginata $\left(\chi^{2}=0.27, \mathrm{df}=1,60 ; P=0.606\right)$ progeny (Table 4).

\section{Discussion}

Beech bark disease was first reported in Halifax, Nova Scotia, Canada around 1890 and continues to spread throughout the range of American beech (Hewitt, 1914; Erhlich, 1934). Likewise, BBD continues to impact European Beech throughout Europe (Cicák et al., 2006; Cicák and Mihál, 2008). Despite the ecological importance of this disease (Houston, 1994b; Garnas et al., 2011a,b), certain aspects of BBD biology and epidemiology remain largely unexplored, including the mating strategies of $N$. ditissima, 
$N$. faginata, and N. coccinea. Previous studies regarding the thallism of $N$. ditissima and N. faginata (El-Gholl et al., 1986; Krüger, 1973; Cotter and Blanchard, 1978) were either inconclusive or sample limited, and as such, the role of mating strategy in the BBD pathosystem in the U.S. has historically been uncertain. Thallism is particularly important to the BBD pathosystem given that at least two dominant, sexually reproducing pathogens are causal agents of the disease in both the U.S. and Europe. Both can colonize single trees and, in some instances, have been found to co-occur within the same 2.5 inch diameter bark disk (Kasson and Livingston, 2009).

Neonectria faginata has been previously reported as the dominant pathogen in the BBD system in North America (Houston, 1994; Kasson and Livingston, 2009). Mating strategy may have provided one explanation for its dominance, but as shown in this study, both $N$. faginata and $N$. ditissima are heterothallic fungi based on limited sampling. Therefore, the possibility of any advantage that homothallism might confer is eliminated, indicating that $N$. faginata is likely dominant due to an increased level of virulence, other advantageous traits, or some combination of these factors.

Despite having resolved the mating strategy of these fungi, the potential overrepresentation of either $N$. ditissima or $N$. faginata in previous studies may be due to perithecia-dependent sampling bias. For example, environmental conditions (e.g. $\mathrm{RH}$, temperature) and time required for perithecia production may significantly differ between these two fungi. Given potential seasonal differences in fruiting, sampling at a single time point in the year could potentially yield a community not representative of the relative abundance of the Neonectria spp. present. Additionally, casual observations of N. ditissima on non-beech hosts can reveal very limited to no perithecia occurring at a 
given infection site. This may be in part due to host susceptibility, resulting in more or less necrotic tissue, which is generally considered favorable for perithecia production by necrotrophic fungi.

Host specificity may develop through the evolution of mating barriers among strains of a single species occurring on differing hosts. Results from this study have demonstrated a lack of reproductive barriers among $N$. ditissima strains from several plant hosts. All pairings of either MAT1-1-1 or MAT1-2-1 strains from different plant hosts resulted in perithecia formation. While a lack of host specificity by $N$. ditissima has been previously demonstrated using pathogenicity assays (Lortie, 1969; $\mathrm{Ng}$ and Roberts, 1974; Barnard et al., 1988; Plante and Bernier, 1997), mating among $N$. ditissima strains infecting co-occurring tree species had not been previously tested until now. Given the lack of reproductive barriers, evolution of host specificity is limited by obligate outcrossing among these co-occurring strains.

The phylogenetic analyses using MAT gene sequences were found to be in agreement with previously resolved relationships demonstrated using EF1- $\alpha, \mathrm{RPB} 2$, and $\beta$-tubulin (Castlebury et al., 2006). This finding confirms the utility of MAT genes in resolving relationships among Neonectria and allied fungi. Additionally, N. ditissima isolates from different plant hosts included in the phylogeny form a single lineage providing additional evidence for the lack of reproductive barriers. The amino acid alignments visualized protein sequence divergence potentially contributing to mating barriers among these closely related species that were confirmed by inter-species mating assays. Using such methods to test intraspecies MAT gene diversity among these and closely allied fungi may prove valuable for broader surveys. 
Limited amplification of non-mating type proteins was observed among several MAT primer - species combinations for isolates of the opposite mating type. However, in all cases, the sequences were found to be larger than the expected size of the target product. Given that such issues can lead to erroneous conclusions, electrophoresis gels should be run to 100 bp resolution and additionally, PCR conditions should be optimized for the target species.

In this study, we confirm heterothallism and characterized the MAT idiomorphs of N. ditissima, N. faginata, $N$. coccinea and several other members of Nectriaceae. These findings provide additional insight into characteristics that may shape the community and population dynamics of the beech bark disease complex and its causal agents. Additional studies are needed to further characterize sexual reproduction of $N$. ditissima, N. faginata, and N. coccinea in their respective BBD systems. These efforts include: 1) identifying differing environmental factors required for perithecia production among Neonectria spp.; 2) characterizing variability in sexual reproduction by $N$. ditissima across host substrates; 3 ) assessing the potential for interspecific hybridization between closely related Neonectria spp. found co-occurring on beech and other hosts but excluded from this study; and 4) comprehensive screening of additional isolates from populations not sampled in this study to assess intraspecies MAT gene diversity and uncover possible intraspecies mating barriers. 


\section{Literature Cited}

Alexopolous, C.J., Mims, C.W., Blackwell, M., 1996. Introductory Mycology, $4^{\text {th }}$ ed. John Wiley \& Sons, Inc, New York.

Barnard, E.L., El-Gholl, N.E., Gilly, S.P., 1988. Comparative spore morphology and pathogenicity of four Florida isolates of Nectria galligena. Plant Disease 72:973976.

Booth, C., 1967. Nectria galligena. CMI Descriptions of Pathogenic Fungi and Bacteria $147: 1-2$

Cale, J.A., Garrison-Johnston, M.T., Teale, S.A., Castello, J.D., 2017.Beech bark disease in North America: Over a century of research revisited. Forest Ecology and Management 394: 86-103.

Carbone, I., Kohn, L.M., 1999. A method for designing primer sets for speciation studies in filamentous fungi. Mycologia 91: 553-556.

Castlebury, L.A., Rossman, A.Y., Hyten, A.S., 2006. Phylogenetic relationships of Neonectria/Cylindrocarpon on Fagus in North America. Canadian Journal of Botany 84: 1417-1433.

Chang, S., Staben, C., 1994. Directed replacement of $\mathrm{mt} \mathrm{A}$ by $\mathrm{mt} \mathrm{a-1} \mathrm{effects} \mathrm{a} \mathrm{mating}$ type switch in Neurospora crassa. Genetics 138:75-81

Chapela, I.H., Boddy, L., 1988. Fungal colonization of attached beech branches. II. 
Spatial and temporal organization of communities arising from latent invaders in bark and functional sapwood, under different moisture regimes. New Phytologist 110:47-57.

Cicák, A., Mihál, I., 2008. Current state of beech bark necrotic disease in Southern Poland. Journal of Forest Science 54:459-464.

Cicák, A., Mihál, I., Tsakov, H., Petkov, P., 2006. Actual status of the beech bark necrotic disease in North Western Bulgaria. Journal of Forest Science 52:226232.

Cotter, H.V.T., Blanchard, R.O., 1978. Heterothallism in Nectria coccinea var. faginata. Mycologia 70:697-700.

Coppin, E., Debuchy, R., Arnaise, S., Picard, M., 1997. Mating types and sexual development in filamentous ascomycetes. Microbiology and Molecular Biology Reviews 61:411-428.

Crane, P.E., Hopkins, A.J.M., Dick, M.A., Bulman, L.S., 2009. Behavior of Neonectria fuckeliana causing a pine canker disease in New Zealand. Canadian Journal of Forest Research 39:2119-2128.

Debuchy, R., Coppin, E., 1992. The mating types of Podospora anserina: functional analysis and sequence of the fertilization domains. Molecular and General Genetics 233:113-121.

Debuchy, R., Turgeon, B.G., 2006. Mating-type structure, evolution, and function in Euascomycetes. The Mycota I: Growth, Differentiation and Sexuality, SpringerVerlag, Berlin. 293-323.

Debuchy, R., Berteaux-Lecellier, V., Silar, P., 2010. Mating systems and sexual 
morphogenesis in ascomycetes. In Cellular and molecular biology of filamentous fungi. American Society of Microbiology. 501-535.

Deng, C.H., Scheper, R.W., Thrimawithana, A.H., Bowen, J.K. 2015. Draft genome sequences of two isolates of the plant-pathogenic fungus Neonectria ditissima that differ in virulence. Genome Announcements 3.

Dukes, J.S., Pontius, J., Orwig, D.A., Garnas, J.R., Rodgers, V.L., Brazee, N., Cooke, B., Theoharides, K.A., Stange, E.E., Harrington, R., Ehrenfeld, J., Gurevitch, J., Lerdau, M., Stinson, K., Wick, R., Ayres, M., 2009. Responses of insect pests, pathogens, and invasive plant species to climate change in the forests of northeastern North America: what can we predict? Canadian Journal of Forest Research 39:231-248.

Ehrlich, J., 1934. The beech bark disease, a Nectria disease of Fagus, following Cryptococcus fagi (Baer.). Canadian Journal of Research 10: 593-692.

El-Gholl, N.E., Barnard, E.L., Schroeder, R.A., 1986. Homothallism in Nectria galligena. Canadian Journal of Botany. 64: 902-903.

Ferreira, A.V., An, Z., Metzenberg, R.L., Glass, N.L., 1998. Characterization of mat A-2, mat A-3 and deltamatA mating-type mutants of Neurospora crassa. Genetics 148:1069-1079.

Garnas, J.R., Houston, D.R., Ayres, M.P., Evans, C., 2011. Disease ontogeny overshadows effects of climate and species interactions on population dynamics in a nonnative forest disease complex. Ecography 35:412-421.

Garnas, J.R., Ayres, M.P., Liebhold, A.M., Evans, C. 2011. Subcontinental impacts of 
an invasive tree disease on forest structure and dynamics. Journal of Ecology 99:532-541.

Glémin, S., Galtier, N., 2012. Genome evolution in outcrossing versus selfing versus asexual species. Methods in Molecular Biology 855:311-335.

Gómez-Cortecero, A., Harrison, R.J., Armitage, A.D., 2015. Draft genome sequence of a European isolate of the apple canker pathogen Neonectria ditissima. Genome Announcements 3 .

González, C.D., Chaverri, P., 2017. Corinectria, a new genus to accommodate Neonectria fuckeliana and C. constricta sp. nov. from Pinus radiata in Chile. Mycological Progress 16:1015-1027.

Gurevich, A., Saveliev, V., Vyahhi, N., Tesler, G., 2013. QUAST: quality assessment tool for genome assemblies. Bioinformatics 29:1072-1075.

Hendry, S.J., Boddy, L., Lonsdale, D., 2002. Abiotic variable effect differential expression of latent infections in beech (Fagus sylvatica). New Phytologist 155:449-460.

Hewitt, C.G., 1914. Note on the occurrence of the felted beech coccus Cryptococcus fagi (Baerens) Dougl. in Nova Scotia. The Canadian Entomologist 46:15-16.

Houston, D.R., Mahoney, E.M., McGauley, B.H., 1987. Beech bark disease: association of Nectria ochroleuca in W.VA., PA, and Ontario. Phytopathology 77:1615.

Houston, D.R., 1994a. Temporal and spatial shift within the Nectria pathogen complex associated with beech bark disease of Fagus grandifolia. Canadian Journal of Forest Research 24:960-968.

Houston, D.R., 1994b. Major new tree disease epidemics: beech bark disease. Annual 
Review of Phytopathology 32:75-87.

Kasson, M.T., Livingston, W.H., 2009. Spatial distribution of Neonectria species associated with beech bark disease in northern Maine. Mycologia 101:190-195.

Kasson, M.T.K., Livingston, W.H., 2012. Relationships among beech bark disease, climate, radial growth response and mortality of American beech in northern Maine, USA. Forest Pathology. 42:199-212.

Koren, S., Walenz, B.P., Berlin, K., Miller, J.R., Bergman, N.H., Phillippy, A.M., 2017. Canu: scalable and accurate long-read assembly via adaptive k-mer weighting and repeat separation. Genome Research 27:722-736.

Kronstad, J.W., Staben, C., 1997. Mating type in filamentous fungi. Annual Review of Genetics 31:245-276.

Krüger, J., 1973. Zur genetik von Nectria galligena Bres. Journal of Phytopathology 79: $320-342$.

Larkin, M.A., Blackshields, G., Brown, N.P., Chenna, R., McGettigan, P.A., McWilliam, H., Valentin, F., Wallace, I.M., Wilm, A., Lopez, R., Thompson, J.D., Gibson, T.J., Higgins, D.G., 2007. Clustal W and Clustal X version 2.0. Bioinformatics 23:2947-2948.

Lohman, M.L., Watson, A.J., 1943. Identity and host relations of Nectria species associated with diseases of hardwoods in the Eastern States. Lloydia 6: 77-108.

Loman, N.J., Quick, J., Simpson, J.T., 2015. A complete bacterial genome assembled de novo using only nanopore sequencing data. Nature Methods 12:733-735.

Lopes, A., Linaldeddu, B.T., Phillps, A.J., Alves, A., 2018. Mating type gene analyses in 
the genus Diplodia: From cryptic sex to cryptic species. Fungal Biology 122: 629638.

Lortie, M., Kuntz, J.E., 1963. Ascospore discharge and conidium release by Nectria galligena Bres. under field and laboratory conditions. Canadian Journal of Botany 41:1203-1210.

Lortie, M., 1969. Inoculation of Nectria galligena on northern hardwoods. Laval University Forest Research Foundation 13:1-29.

McCullough, D., Wieferich, J., 2015. Beech bark disease in Michigan: spread of the advancing front and stand-level impacts. Forest Health Monitoring: National Status, Trends, and Analysis 2014, USDA Forest Service, Southern Research Station, Asheville, NC. 125- 132.

McLaughlin, J., Greifenhagen, S., 2012. Beech bark disease in Ontario: a primer and management recommendations. Forest Research Note - Ontario Forest Research Institute, Sault Ste Marie, Ontario, Canada.

Ng, K.W., Roberts, E.T., 1974. Pathogenicity of Nectria galligena. Plant Pathology 23: 49-50.

Plante, F., Bernier, L., 1997. Variability of virulence of Nectria galligena towards northern hardwoods. European Journal of Plant Pathology 27:261-272.

Pöggeler, S., Kück, U., 2000. Comparative analysis of the mating-type loci from Neurospora crassa and Sordaria macrospora: identification of novel transcribed ORFs. Molecular and General Genetics 263:292-301.

Rehner, S., 2001. Primers for Elongation Factor 1- $\alpha$ (EF1- $\alpha)$. Insect Biocontrol Laboratory: USDA, ARS, PSI. 
Salgado-Salazar, C., Crouch, J.A., 2019. Genome resources for the stem and bark canker pathogens Corinectria fuckeliana, Neonectria hederae, and N. punicea. Plant Disease 103: 389-391.

Saupe, S., Stenberg, L., Shiu, K.T., Griffiths, A.J., Glass, N.L. 1996. The molecular nature of mutations in the mt A-1 gene of the Neurospora crassa A idiomorph and their relation to mating-type function. Molecular and General Genetics 250:115-22

Simão, F.A., Waterhouse, R.M., loannidis, P., Kriventseva, E.V., Zdobnov, E.M., 2015. BUSCO: assessing genome assembly and annotation completeness with singlecopy orthologs. Bioinformatics 31:3210-3212.

Spaulding, P., Grant, T.J., Ayers, T.T., 1936. Investigations of Nectria diseases in hardwoods of New England. Journal of Forestry 34:169-179.

Stanke, M., Diekhans, M., Baertsch, R., Haussler, D., 2008. Using native and syntenically mapped cDNA alignments to improve de novo gene finding. Bioinformatics 24:637-644.

Stecher, G., Tamura, K., Kumar, S., 2020. Molecular Evolutionary Genetics Analysis (MEGA) for macOS. Molecular Biology and Evolution.

Thomsen, M., Buchwald, N.F., Hauberg, P., 1949. Angreb af Cryptococcus fagi, Nectria galligena og andre parasiter paa bog i Danmark 1939-43. Det Forstlige Forsogsvaesen, 18: 97-326.

Turgeon, B.G., 1998. Application of mating type gene technology to problems in fungal biology. Annual Review of Phytopathology 36:115-137.

Vallone, P., Butler, J., 2004. AutoDimer: A screening tool for primer-dimer and hairpin 
structures. BioTechniques 37:226-231.

van Diepen, L.T., Frey, S.D., Landis, E.A., Morrison, E.W., Pringle, A., 2017. Fungi exposed to chronic nitrogen enrichment are less able to decay leaf litter. Ecology. 98: 5-11.

Walker, B.J., Abeel, T., Shea, T., Priest, M., Abouelliel, A., Sakthikumar, S., Cuomo, C.A., Zeng, Q., Wortman, J., Young, S.K., Earl, A.M., 2014. Pilon: an integrated tool for comprehensive microbial variant detection and genome assembly improvement. PLOS ONE 9.

White, T.J., Bruns, T., Lee, S., Taylor, J., 1990. Amplification and direct sequencing of fungal ribosomal RNA genes for phylogenetics. PCR Protocols-A Guide to Methods and Applications 18:315-322.

Yun, S.H., Arie, T., Kaneko, I., Yoder, O.C., Turgeon, B.G., 2000. Molecular organization of mating type loci in heterothallic, homothallic, and asexual Gibberella/Fusarium species. Fungal Genetics and Biology 31:7-20. 


\section{Supplemental Figure}

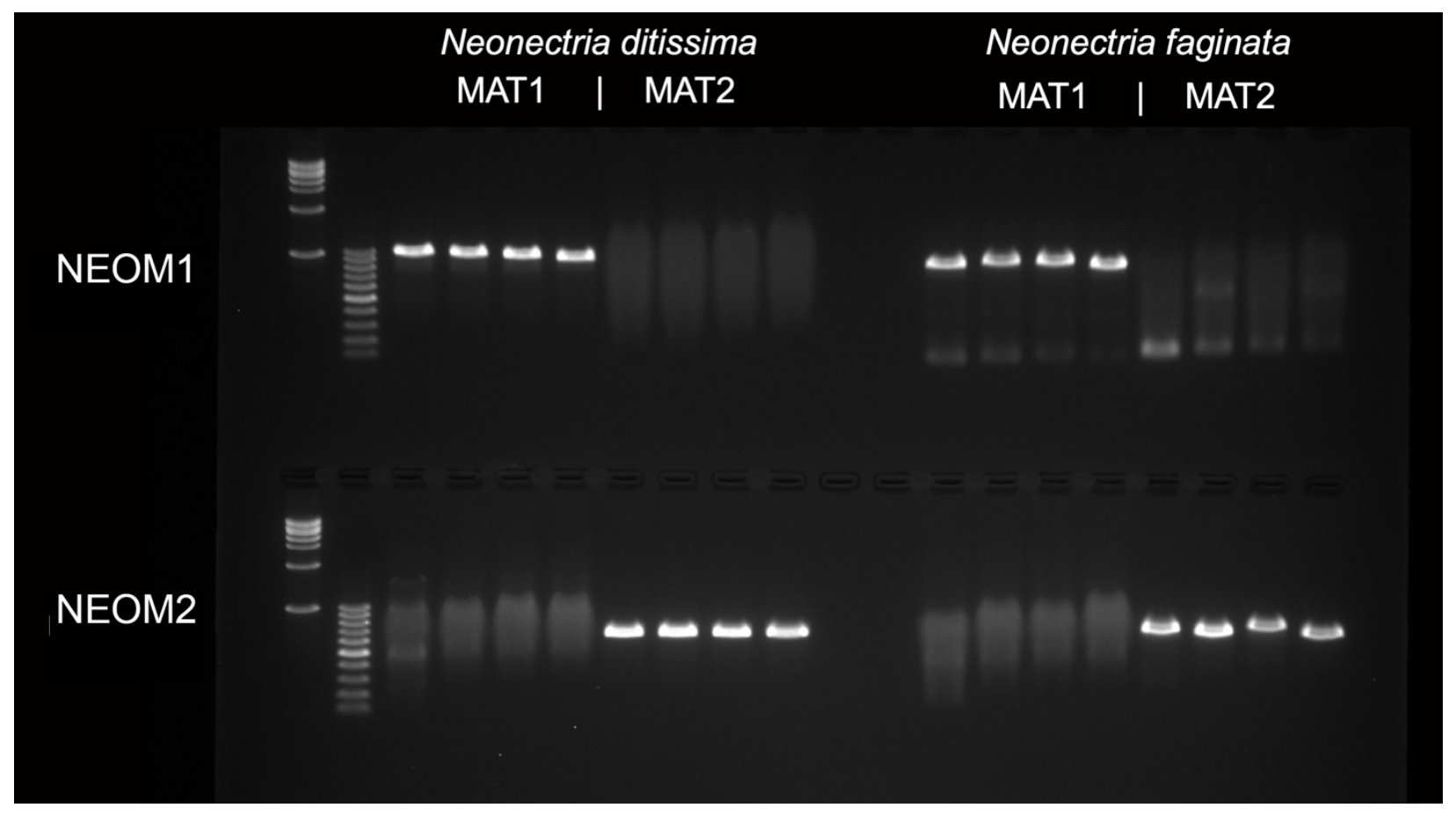

Supplemental Figure 1: Electrophoresis gel photo demonstrating specific amplification of MAT1-1-1 and MAT1-2-1 by the genus-level primer pair (NeoM1f/r and NeoM2f/r) for both $N$. ditissima and $N$. faginata. 


\section{Supplemental Table}

Supplemental Table 1: Non-target amplification identities for primer $x$ species pairings: MAT identities are given with the species names. The approximate product sized expected for specific amplification of the target MAT gene are provided. For instances of multiple non-target amplicons among tested species, the lowest sequence similarity value is given. Non-target protein blastx results with the greatest percent sequence coverage are provided including: query coverage, percent identity, and $\mathrm{NCBI}$ accession numbers.

\begin{tabular}{|c|c|c|c|c|c|c|c|}
\hline \multirow[b]{2}{*}{$\begin{array}{l}\text { DNA Template } \\
\quad(\text { MAT ID) }\end{array}$} & \multirow[b]{2}{*}{$\begin{array}{l}\text { Primer } \\
\text { Pair }\end{array}$} & \multirow{2}{*}{$\begin{array}{l}\text { Approx. } \\
\text { Target } \\
\text { Product } \\
\text { Size (bp) }\end{array}$} & \multirow{2}{*}{$\begin{array}{l}\text { Approx. } \\
\text { Non-Target } \\
\text { Product } \\
\text { Size (bp) }\end{array}$} & \multirow{2}{*}{$\begin{array}{c}\text { Sequence } \\
\text { Similarity Among } \\
\text { Non-Target } \\
\text { Amplicons } \\
\end{array}$} & \multicolumn{3}{|c|}{ Non-Target blastx Results } \\
\hline & & & & & $\begin{array}{c}\text { Query } \\
\text { Coverage }\end{array}$ & $\begin{array}{c}\% \\
\text { Identity }\end{array}$ & $\begin{array}{c}\mathrm{NCBI} \\
\text { Accession }\end{array}$ \\
\hline N. coccinea (M2) & $\mathrm{NfM} 1 \mathrm{f} / \mathrm{r}$ & 530 & 650 & $94 \%$ & $88.0 \%$ & $90.0 \%$ & KPM41412 \\
\hline N. neomacrospora (M2) & $\mathrm{NfM} 1 \mathrm{f} / \mathrm{r}$ & 530 & 1000 & $N / A$ & $29.0 \%$ & $83.5 \%$ & KPM36460 \\
\hline C. aff. fuckeliana (M2) & $\mathrm{NfM} 1 \mathrm{f} / \mathrm{r}$ & 530 & 1000 & $N / A$ & $32.0 \%$ & $75.2 \%$ & KPM45843 \\
\hline N. coccinea (M1) & $\mathrm{NfM} 2 \mathrm{f} / \mathrm{r}$ & 610 & 1150 & $81 \%$ & $51.0 \%$ & $68.2 \%$ & KPM37850 \\
\hline C. aff. fuckeliana (M1) & $\mathrm{NfM} 2 \mathrm{f} / \mathrm{r}$ & 610 & 800 & $82 \%$ & $45.0 \%$ & $83.5 \%$ & KPM46131 \\
\hline N. coccinea (M1) & NeoM2f/r & 610 & 1200 & $87 \%$ & $44.0 \%$ & $74.8 \%$ & KPM37850 \\
\hline N. neomacrospora (M1) & NeoM2f/r & 610 & 1200 & $76 \%$ & $50.0 \%$ & $35.7 \%$ & KPM37850 \\
\hline
\end{tabular}




\title{
CHAPTER 3: Corinectria gaudineerii sp. nov., a novel nectriaceous fungus associated with high elevation Picea rubens in the Appalachian Mountains
}

\begin{abstract}
The Nectriaceae is a family of fungi including many phytopathogens capable of producing cankers on hardwood and conifer host species. Among these nectriaceous fungi are members of the recently circumscribed genus Corinectria. Members of Corinectria are closely related to Neonectria, but unlike Neonectria whose members primarily infect hardwood tree species, Corinectria spp. are found exclusively on conifer tree species as canker pathogens or hemibiotrophs. In this study, Corinectria gaudineerii sp. nov. is described as a new member of the recently circumscribed genus Corinectria. C. gaudineerii is the fourth species described for this genus but represents the first to be described occurring in the central Appalachian Mountains on the bark of dead Picea rubens (red spruce). In this study, we provide phylogenetic evidence using five loci (ITS, LSU, BTUB, EF1, ACT) to characterize C. gaudineerii as a new species. Additionally, field surveys recovered C. gaudineerii from four geographically separated spruce forests in WV and VA. Finally, live- and excised-stem assays were conducted to determine the pathogenic and saprophytic potential, revealing C. gaudineerii to be a hemibiotroph and possibly, a weak canker pathogen. Together, these results provide evidence to support the description of a novel species of Corinectria associated with dead red spruce in central Appalachia.
\end{abstract}




\section{Introduction}

Corinectria is a recently circumscribed genus in the Nectriaceae that formerly belonged to but was phylogenetically distinct from the closely related Neonectria (González and Chaverri, 2017). This genus consists of three formally described species: C. fuckeliana ([C. Booth] C. González \& P. Chaverri), C. constricta (C. González \& P. Chaverri), and C. tsugae ([W. Gams] C. González \& P. Chaverri). All members of Corinectria are hemibiotrophic and are associated with stem cankers on diverse coniferous hosts including Picea, Abies, Pinus, and Larix with distribution in both the northern and southern hemispheres. C. fuckeliana (formerly Neonectria fuckeliana), which is among the most well studied Corinectria species, has been associated with species of the aforementioned host genera as a weak pathogen, saprophyte, and endophyte (Huse, 1981, Rolls-Hansen and Rolls-Hansen, 1979, Vasiliauskas and Stenlid, 1998). C. tsugae has been only reported on Tsuga heterophylla (Raf.) Sarg. in western North America (Williams, 1987). More recently, previous reports of $C$. fuckeliana infecting Pinus radiata D.Don in Chile (Gadgil et al., 2003, Dick and Crane, 2009, Crane et al., 2009) were re-evaluated and determined to be a separate species leading to the circumscription of $C$. constricta (González and Chaverri, 2017). To date, all species infected and/or associated with Corinectria members have been coniferous species, and none have been confirmed on any trees native to eastern North America.

In December of 2018, perithecia were observed extruding from bark along the trunk of toppled dead understory red spruce (Picea rubens Sarg.) trees on Gaudineer Knob in the Monongahela National Forest of West Virginia, USA. This discovery was 
reported in a Chapters 1 and 2 where phylogenetic results indicated this species to be divergent from all previously described Corinectria spp. Further, this putatively novel Corinectria sp. was exclusive to red spruce trees at three geographically separate locations in West Virginia and Virginia, but no formal description of this species was made nor were any morphological characters compared with previously described Corinectria spp.

Red spruce has been a primary target for restoration in the Appalachian Mountains over the last few decades following several decades of decline. Extensive surveys of declining or dead red spruce trees were conducted to identify plant pathogens potentially contributing to this decline, but none reported "Nectria"-like fungi associated with red spruce (Johnson and McLaughlin, 1986, Bruck, 1989). Given that this Corinectria species was identified at several locations in central Appalachia, this fungus may represent a newly introduced pathogen that has only recently encountered native red spruce. Otherwise, a native fungus has significantly increased in incidence from historic undetectable levels due to unique stressors that are predisposing trees to fungal invasion, similar to BBD following the introduction of beech scale although no scale insect has been observed.

To further investigate this putatively novel Corinectria sp., we sought to determine the identity of this Corinectria sp. using phylogenetic and morphological comparisons with previously described species. This was important as the lack of previous reports of this fungus despite extensive red spruce surveys might indicate the introduction of a new phytopathogen. We next sought to conduct surveys to determine the ecology of this fungus by screening red spruce stands throughout the central 
Appalachian Mountains, and by doing so, determine the threat this new species might pose to the red spruce forests. Finally, we sought to test the pathogenic and saprophytic capacity of this fungus using live- and excised-stem assays. Together these provide the baseline for characterizing the biology and ecology of this novel Corinectria sp. across red spruce forests of central Appalachia. Such investigations are vital to identify potential threats to management and restoration efforts of red spruce.

\section{Materials and Methods}

\section{Survey approach}

Sampling areas with large populations of red spruce were selected in West Virginia and Virginia. These sites were part of a broader survey of Nectraiaceae across the central Appalachian Mountains (Chapter 1). These sites included sampling areas near Mount Rogers (MR) and Whitetop Mountain (WT) of the Jefferson National Forest in VA and areas near Cranberry Wilderness (CR) and Gaudineer Knob (GK) of the Monongahela National Forest in WV (Figure 1; Supplemental Table 1). The United States Forest Service provided a permit for sampling on the Mon. National Forest (FS 2400-8) and written permission were given to collect samples without a special use permit on the Jefferson National Forest. At each location, declining red spruce stands were identified by the occurrence of five or more outwardly stressed or dead understory or intermediate red spruce trees within a 1/10 acre plot. Within each plot at WT, MR, and $\mathrm{CR}$, tree health status, size class, canopy position, and the presence of perithecia were recorded for all red spruce trees. No data were collected at GK. Tree health statuses included alive ( $A ;<50 \%$ dieback), declining ( $V ;>50 \%$ dieback), and dead ( $D$; loss of fine branches and bark). Size classes were recorded in ten-centimeter 
increments of $0-10 \mathrm{~cm}, 10-20 \mathrm{~cm}$, and $>20 \mathrm{~cm}$ diameter at breast height (DBH). Canopy positions included dominant, co-dominant, intermediate, and suppressed.

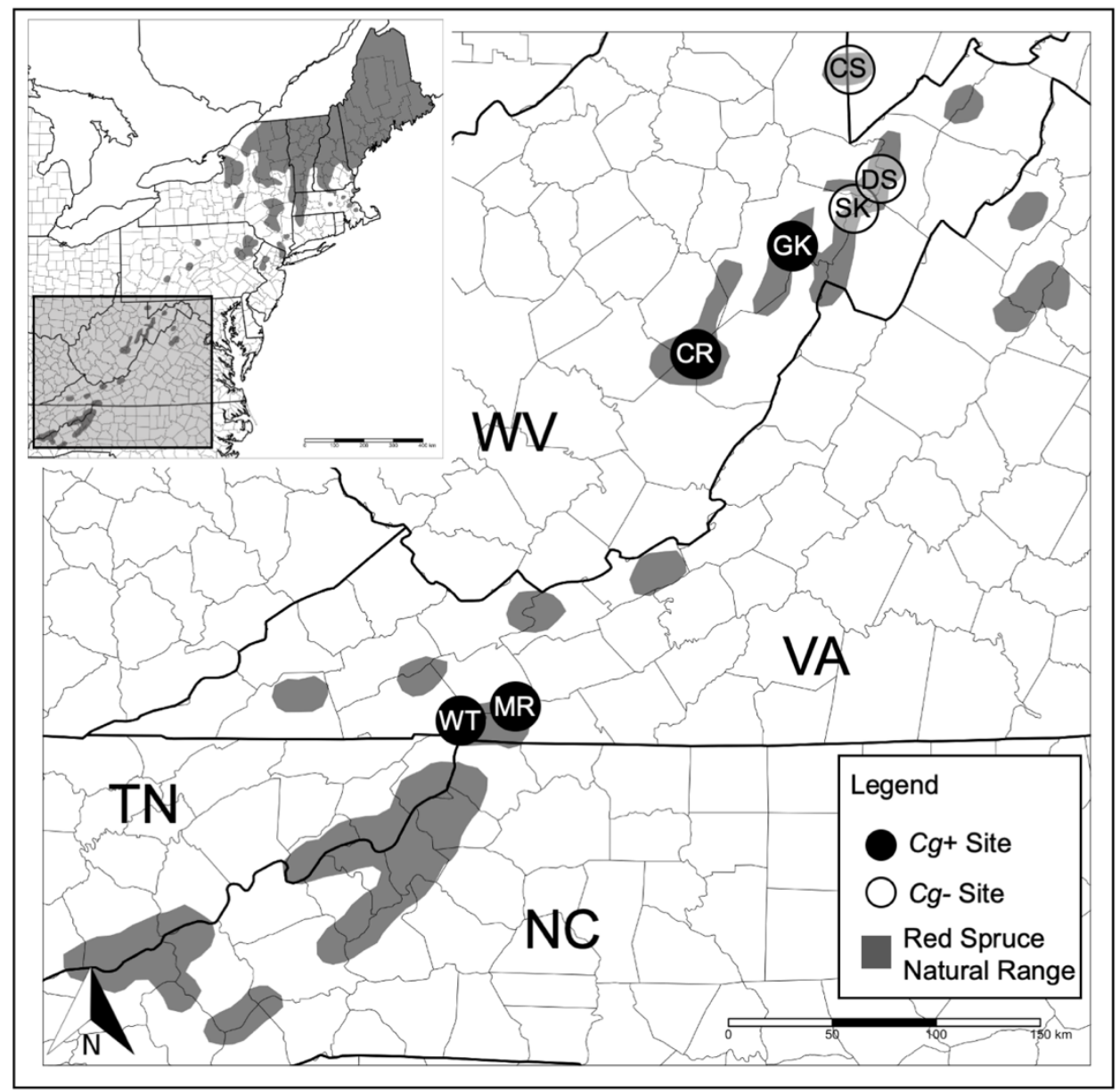

Figure 1: Sampling locations for members of Nectriaceae on red spruce in WV and VA. These include Mount Rogers (MR) and Whitetop Mountain (WT) of the Jefferson National Forest in VA and areas near Cranberry Wilderness (CR) and Gaudineer Knob (GK) of the Monongahela National Forest in WV. Shaded areas represent the natural range of red spruce (Prasad and Iverson 2003).

\section{Sampling method and processing}

A maximum of four bark disks harboring perithecia were excised from red spruce trees with a 1-cm steel punch and stored in 96-well microtiter dishes. All samples were 
stored on ice until they were stored at $-20^{\circ} \mathrm{C}$ in the laboratory until being processed. Sample processing was completed as previously described in Chapter 2. In summary, five perithecia were removed from each bark disk, macerated in $1 \mathrm{ml}$ of sterile $\mathrm{H}_{2} \mathrm{O}$ within a microcentrifuge tube, vortexed for 15 seconds, then $300 \mu \mathrm{l}$ of the spore suspension was spread on glucose-yeast extract agar plates (GYE/A) amended with streptomycin sulfate $(10 \mathrm{mg} / 1000 \mathrm{ml})$ and tetracycline hydrochloride $(100 \mathrm{mg} / 1000 \mathrm{ml})$

antibiotics. Five single ascospore colonies were transferred to a new GYE/A plate within 48 hours. Selected isolates were stored at approximately $-20^{\circ} \mathrm{C}$ on glass filter paper.

\section{DNA Extraction and PCR Protocol}

Suspect Corinectria isolates recovered from field-collected perithecia were grouped and tentatively identified based on colony morphology. Genomic DNA extractions were performed for selected isolates using a modified Wizard® kit (Promega, Madison, WI, USA) and suspended in $75 \mu \mathrm{l} \mathrm{Tris-EDTA} \mathrm{(TE)} \mathrm{buffer} \mathrm{(Amresco,}$ Solon, OH, USA) (Short et al. 2015). The fungal bar-coding genes internal transcribed spacer region (ITS), translation elongation factor 1-alpha (EF1), 28S rDNA (LSU), $\beta$ tublin (BTUB), and actin (ACT) were PCR amplified in $25 \mu$ reactions containing $12.5 \mu \mathrm{l}$ Bioline PCR Master Mix (Bioline USA Inc, Taunton, MA), $10.0 \mu \mathrm{H}_{2} \mathrm{O}, 1.5 \mu$ purified DNA, and $1.0 \mu \mathrm{l}$ each of forward and reverse primers (Integrated DNA Technologies, Coralville, IA, USA). All primers, protocols, and their sources are outlined in Supplemental Table 2. PCR products were visualized via gel electrophoresis, and positive reactions were purified using ExoSAP-IT (Affymetrix, Santa Clara, CA, USA) according to the manufacturer's recommendations. Purified PCR products were Sanger 
sequenced with both forward and reverse PCR primers (Eurofins, Huntsville, AL, USA).

\section{Phylogenetic analyses}

Phylogenetic analyses were conducted as described in Chapter 2 and are summarized below. Assessment and clipping of sequences were completed with CodonCode Aligner v. 5.1.5, then manual corrections for nucleotide misreads were performed. To resolve the identity of recovered Corinectria isolates, single-gene and concatenated five-gene phylogenetic trees were constructed using additional reference sequences for all described members of Corinectria along with selected Neonectria members to serve as outgroup taxa (Table 1). Alignments were completed via MAFFT (Katoh and Stanley, 2013) on the Guidance 2.0 server (Landan and Graur, 2008, Sela et al., 2015), and all resides with Guidance scores $<0.5$ were masked (Macias et al., 2020). Aligned single gene sequences were concatenated using the web tool FaBox (Villesen 2007).

Maximum-likelihood analyses were performed in MEGA v10.1.7 (Stecher et al., 2020), and Bayesian inference (BI) analyses were performed using MrBayes v. 3.2.7 (Ronquist et al., 2012). Model Test AICc scores in MEGA were used to select the best fit substitution model for ML analyses. All ML analyses included 1000 bootstrap replicates. For $\mathrm{BI}$ analyses, MrBayes was allowed to select the best fit nucleotide selection model, but Model test AICc scores were used to select the rate of substitution. All BI analyses ran until the standard deviation of split frequencies fell below 0.01 and then checked for convergence in Tracer v. 1.7.1 (Rambaut et al., 2018). Trees were edited using FigTree v. 1.4.4 (Rambaut, 2017) and Adobe Illustrator v. 24.1 
Table 1: Accession table for sequences used in this study. Sequences were either generated here or acquired from NCBI. Host species and location are also provided where available.

\begin{tabular}{|c|c|c|c|c|c|c|c|c|}
\hline Species & Strain ID & Host & Locality & BT & LSU & EF1 & ITS & ACT \\
\hline $\begin{array}{l}\text { Neonectria } \\
\text { ditissima }\end{array}$ & NdFg001 & Fagus grandifolia & $\begin{array}{c}\text { Randolph County, WV } \\
\text { USA }\end{array}$ & TBD & TBD & TBD & TBD & TBD \\
\hline $\begin{array}{l}\text { Neonectria } \\
\text { faginata }\end{array}$ & $\mathrm{NfFg007}$ & Fagus grandifolia & Swain County, NC, USA & TBD & TBD & TBD & TBD & TBD \\
\hline $\begin{array}{l}\text { Neonectria } \\
\text { magnoliae }\end{array}$ & NmMf001 & Magnolia fraseri & $\begin{array}{c}\text { Randolph County, WV, } \\
\text { USA }\end{array}$ & TBD & TBD & TBD & TBD & TBD \\
\hline $\begin{array}{l}\text { Corinectria } \\
\text { gaudneerii }\end{array}$ & CgPr004 & Picea rubens & $\begin{array}{c}\text { Randolph County, WV, } \\
\text { USA }\end{array}$ & TBD & TBD & TBD & TBD & - \\
\hline $\begin{array}{l}\text { Corinectria } \\
\text { gaudneerii }\end{array}$ & CgPr007 & Picea rubens & $\begin{array}{c}\text { Randolph County, WV, } \\
\text { USA }\end{array}$ & TBD & TBD & TBD & TBD & TBD \\
\hline $\begin{array}{l}\text { Corinectria } \\
\text { gaudneerii }\end{array}$ & CgPr009 & Picea rubens & $\begin{array}{c}\text { Randolph County, WV } \\
\text { USA }\end{array}$ & TBD & TBD & TBD & TBD & TBD \\
\hline $\begin{array}{l}\text { Corinectria } \\
\text { gaudneerii }\end{array}$ & CgPr002 & Picea rubens & $\begin{array}{c}\text { Grayson County, Virginia, } \\
\text { USA }\end{array}$ & TBD & TBD & TBD & TBD & TBD \\
\hline $\begin{array}{l}\text { Corinectria } \\
\text { gaudneerii }\end{array}$ & CgPr010 & Picea rubens & $\begin{array}{c}\text { Pocahontas County, WV } \\
\text { USA }\end{array}$ & TBD & TBD & TBD & TBD & TBD \\
\hline $\begin{array}{l}\text { Corinectria } \\
\text { gaudneerii }\end{array}$ & CgPr011 & Picea rubens & $\begin{array}{c}\text { Pocahontas County, WV } \\
\text { USA }\end{array}$ & TBD & TBD & TBD & TBD & TBD \\
\hline $\begin{array}{l}\text { Corinectria } \\
\text { gaudneerii }\end{array}$ & CgPr008 & Picea rubens & $\begin{array}{c}\text { Grayson County, Virginia, } \\
\text { USA }\end{array}$ & - & TBD & TBD & TBD & - \\
\hline $\begin{array}{l}\text { Corinectria } \\
\text { tsugae }\end{array}$ & CBS 788.69 & Tsuga heterophylla & Canada & KM232020.1 & MH871201.1 & - & KM231763.1 & KM231147.1 \\
\hline $\begin{array}{l}\text { Corinectria } \\
\text { tsugae }\end{array}$ & CBS 290.70 & Tsuga heterophylla & Canada & - & MH871386.1 & - & MH859632.1 & - \\
\hline $\begin{array}{l}\text { Corinectria } \\
\text { fuckeliana }\end{array}$ & IMI 342668 & Picea sp. & Switzerland & KJ022340.1 & KJ022070.1 & KJ022404.1 & KJ022021.1 & KJ022285.1 \\
\hline $\begin{array}{l}\text { Corinectria } \\
\text { fuckeliana }\end{array}$ & CBS 239.29 & Picea sitchensis & Scotland & DQ789871.1 & HQ840377.1 & JF268748.1 & HQ840386.1 & - \\
\hline $\begin{array}{l}\text { Corinectria } \\
\text { constricta }\end{array}$ & LASBE 266 & Pinus radiata & Chile & KY636.417.1 & - & KY636410.1 & - & KY636427.1 \\
\hline $\begin{array}{l}\text { Corinectria } \\
\text { constricta }\end{array}$ & LASBE 340 & Pinus radiata & Chile & KY636423.1 & - & KY636414.1 & - & KY636433.1 \\
\hline
\end{tabular}




\section{Morphological characterization of Corinectria gaudineerii}

Ascospore and conidia measurements of $C$. gaudineerii were conducted and compared to previously described Corinectria members (González and Chaverri, 2017). Ascospore measurements were completed for three perithecia from each of five bark disks collected from two geographically separated sites (WT = 3 disks; GK $=2$ disks). Spore measurements were completed by squash-mounting a single perithecium on a slide with lactophenol plus cotton blue mountant. Length and width measurements measurements were taken with a Nikon Eclipse E600 compound microscope (Nikon Instruments, Melville, NY, USA) equipped with a Nikon Digital Sight DS-Ri1 microscope camera and Nikon NIS-Elements BR3.2 imaging software for 25 ascospores per perithecium.

Length and width measurements were similarly conducted for microconidia harvested from sporodochial masses on four-to-six week old pure-cultures grown on GYE. Measurements were taken for 50 microconidia harvested from four isolates from WT and two isolates from GK. Macroconidia were not found associated with any included isolates.

\section{Pathogenicity of C. gaudineerii on Picea rubens}

To test the pathogenicity of $C$. gaudineerii, a pathogenicity assay was conducted on 2.5-3.5 year old red spruce seedlings grown from seed collected near Spruce Knob, WV in 2011 and 2015. Corinectria sp. isolates included one recovered from WT in VA (CspPr002) and a second from GK in WV (CspPr004). Isolates were grown in pureculture on GYE media for 10 days at $20^{\circ} \mathrm{C}$ under a $16-8 \mathrm{~h}$ light-dark cycle. Inoculum plugs were cut along the growing edge of the fungal colonies using a $5 \mathrm{~mm}$ diameter 
steel punch. Negative control plugs were similarly cut from a sterile GYE plate. A 1-cm incision was made in the bark tissue with a sterile scalpel to expose the vascular cambium as an inoculation site upon which an inoculum plug was placed. Parafilm was used to cover the inoculation site to minimize desiccation of the inoculum prior to infection. In total, five seedlings were inoculated with isolate CspPr002, five with isolate CspPr004, and five with a sterile agar plug to serve as a negative control. Seedlings were placed on a window seal providing ambient light. Every other day, the seedlings were watered, and their positions were rotated.

At the end of eight weeks, a scalpel was used to remove the bark tissue surrounding the inoculation site. Length and width measurements were taken for all necrotic lesions associated with the inoculation sites. Canker growth was calculated as $=($ length + width $) / 2$. Four bark samples were excised from each canker margin for reisolation of inoculum. All bark samples were surface disinfested by soaking for 14 minutes in a 1:10 commercial bleach-water solution then placed onto GYE/A media. Recovery of inoculation isolates was confirmed morphologically.

\section{Saprophytic capacity of C. gaudineerii on Picea rubens}

Over the course of the survey, perithecia of $C$. gaudineerii were found exclusively on dead red spruce. While this does not exclude the possible pathogenicity of $C$. gaudineerii, the saprophytic state of this fungus was the only state in which the fungus was observed. As such, an excised-stem assay was completed using freshly cut red spruce stems. The ends of each stem were coated in parafilm to limit stem desiccation. Prior to inoculation, each stem was disinfested in a 1:10 commercial bleach solution for 
14 minutes. Inoculum was prepared as previously described, but a 1-cm steel punch was used to cut inoculation plugs.

The assay included four treatments: 1) C. gaudineerii; 2) Neonectria faginata; 3) N. ditissima; and 4) negative control. The two Neonectria treatments served as comparison fungi capable of saprotrophy, although exclusively on hardwood tree species. Inoculations proceeded as a 1-cm diameter steel punch was used to create two inoculum reservoirs on each stem. An inoculum plug was then placed in the reservoir and tape was applied to limit desiccation of the inoculum. Each stem had a single treatment for a total of 8 inoculations for $C$. gaudineerii and 6 inoculations for all other treatments. Once inoculated, stems were placed into separate loosely tied autoclave bags with three tri-fold paper towels soaked with approximately $100 \mathrm{ml}$ of $\mathrm{H}_{2} \mathrm{O}$. All stems were stored in a dark closet. After approximately 4 weeks, stems were removed and processed as described above for measurements and inoculum recovery.

\section{Statistical analyses}

One-way ANOVAs were completed to check for differences in canker size measurements taken in pathogenicity assays with a Tukey-HSD post-hoc test to identify significant pairwise differences. All analyses were completed using the stats v3.6.2 package within $R \vee 3.6 .3$ statistical software ( $R$ Core Team, 2020). P-values $<0.05$ were considered statistically significant. 


\section{Results}

\section{Survey of C. gaudineerii on Picea rubens in central Appalachia}

Across all sites, a total of 312 red spruce trees were surveyed, with $6.7 \%$ (21 of 312) of these trees harboring perithecia that yielded C. gaudineerii, hereafter referred to as $\mathrm{Cg}$. No other perithecia-producing fungi were recovered from collected samples. Specifically, these positive samples represented $11.5 \%$ (17 of 148 ), $4.2 \%$ ( 2 of 48 ), and $1.7 \%$ (2 of 116) of surveyed trees at WT, MR, and CR, respectively. Additionally, three trees were sampled at GK, but no formal plots were established at this site.

Site data were collected at WT, MR, and CR and included size class, tree health, and crown class. All red spruce tree harboring perithecia were dead, but a majority of these trees $(85.7 \%$; 18 of 21$)$ were not significantly decayed as the bark and fine branches of the crown were still intact. Additionally, a majority of $\mathrm{Cg}$-positive trees were found to be suppressed underneath a closed canopy (95.2\%; 20 of 21$)$, and all were of the smallest size class.

\section{Phylogenetic resolution of C. gaudineerii}

Phylogenetic analyses of single genes (ACT, BT, EF1, ITS, LSU) and a five-gene concatenated sequence containing 2,526 positions were conducted to infer the relationship of $\mathrm{Cg}$ with other members of Corinectria. As previously mentioned, these analyses were bolstered by the inclusion of the following three previously described Corinectria species: C. constricta, C. fuckeliana, and C. tsugae (Table 1). Sequence data for all five genes were not available for every included strain, and this is reflected in the single gene phylogenies. All available sequence data were used for concatenated sequences, and all strains were represented by $\geq 3$ single gene sequences with one 
exception, C. tsugae strain CBS 290.70 was only represented by LSU and ITS sequences.

The following results are for relationships with $>70 \%$ bootstrap support within the concatenated phylogeny, and both $\mathrm{ML}$ and $\mathrm{BI}$ values are reported for those relationships (ML/BI) (Figure 2). Disagreements between single-gene and concatenated phylogenies are also noted (Figure 3). All Corinectria spp. were monophyletic $(100 \% / 1.0)$ and sister to included Neonectria spp. C. gaudineerii isolates recovered in this survey formed a monophyletic clade sister to all other previously described Corinectria spp. $(98 \% / 1.0)$, and this was strongly supported $(>77 \% />0.77)$ in all single gene trees aside from the BT phylogeny in which no Corinectria spp. were genealogically exclusive (Figure 3). The only other genealogically exclusive Corinectria spp. in the concatenated tree was C. constricta $(97 \% / 0.98)$, but this was not strongly supported in any of the five single gene trees. 


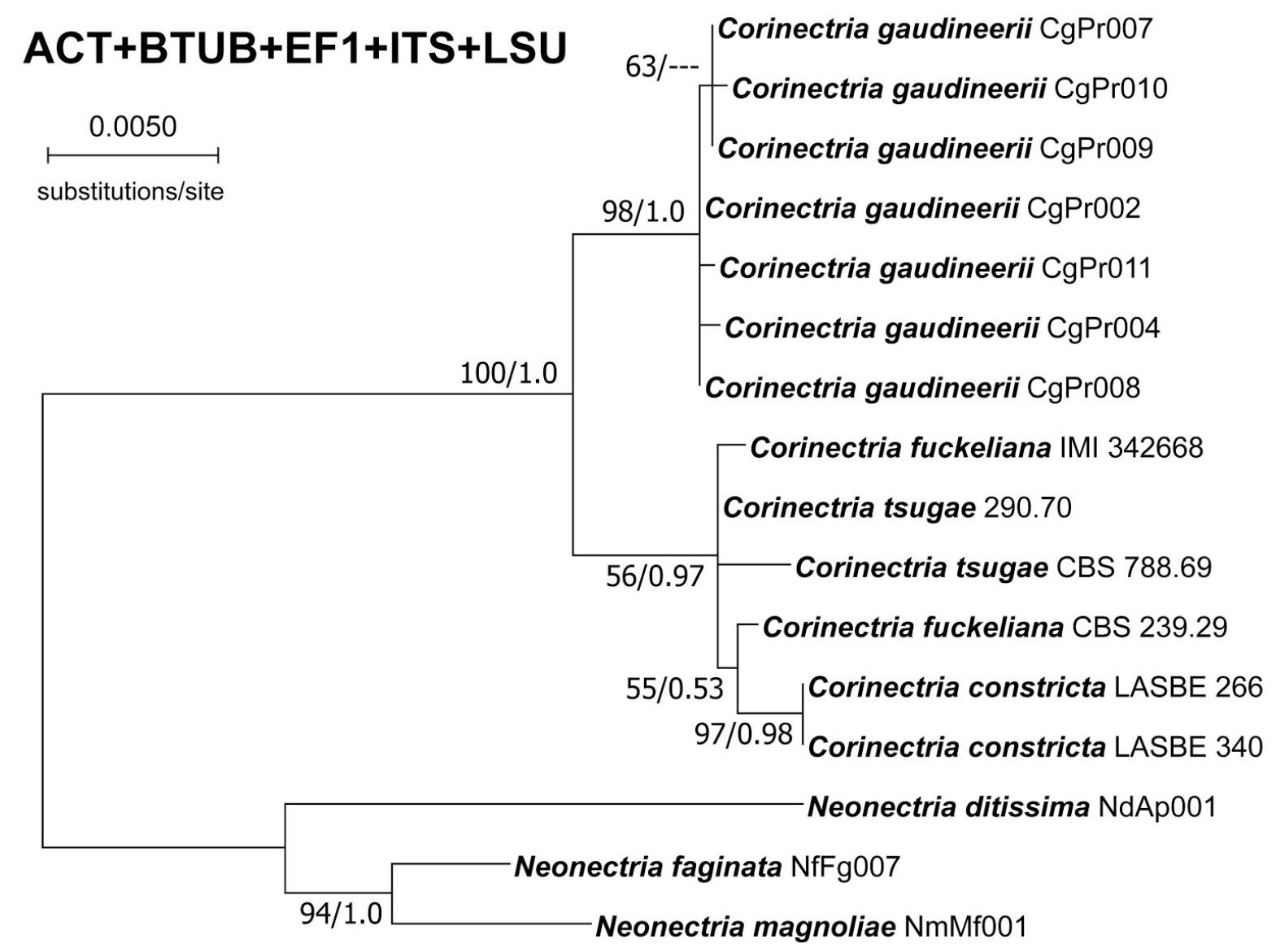

Figure 2: Concatenated sequence (ACT, BTUB, EF1, ITS, LSU) phylogenetic tree of Corinectria spp. and Neonectria spp. outgroups. Topology and branch lengths are from the $M L$ analysis. For each node supported in the ML analysis, bootstrap support and posterior probabilities are indicated (ML/BI). 


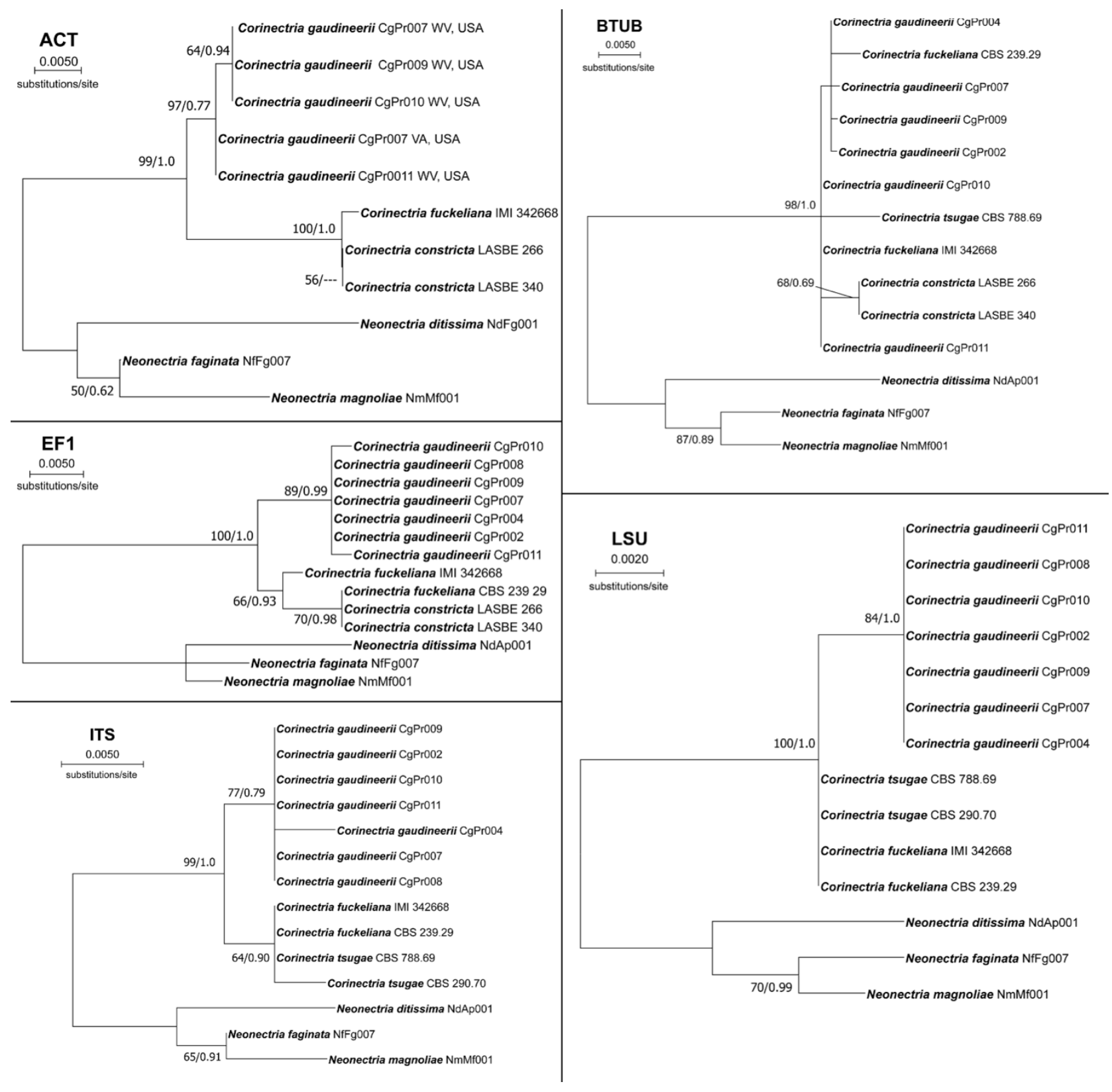

Figure 3: Single-gene (ACT, BTUB, EF1, ITS, LSU) phylogenetic trees of Corinectria spp. and Neonectria spp. outgroups. Topology and branch lengths are from the ML analysis. For each node supported in the ML analysis, bootstrap support and posterior probabilities are indicated (ML/BI). 


\section{Pathogenicity and saprotrophy of C. gaudineerii on Picea rubens}

In the pathogenicity assay on red spruce seedlings, all $\mathrm{Cg}$ inoculations produced a necrotic lesion from which the inoculation isolate was recovered (Figure $4 \mathrm{~A})$. Both $\mathrm{Cg}$ treatments produced similar canker sizes $(p=0.208)$ that were significantly greater than the negative control $(p<0.00001)$. In this case, the negative control had no measurable necrosis, and therefore, was set at $0 \mathrm{~cm}$. While not an included metric in this study, no obvious dieback was observed, and all trees survived to the end of the study.

In the excised stem assay, two additional treatments using isolates of Neonectria faginata and $N$. ditissima were included in addition to a single $\mathrm{Cg}$ and a negative control (Figure 4B). In this case, both $\mathrm{Cg}$ and $\mathrm{N}$. ditissima produced necrotic lesions larger than $N$. faginata and the negative control $(p<0.001)$, which both similarly produced little to no necrosis larger than the inoculation wound $(\sim 1 \mathrm{~cm})(p=0.988)$. Each treatment's inoculation isolate was recovered from all respective areas of necrosis. 


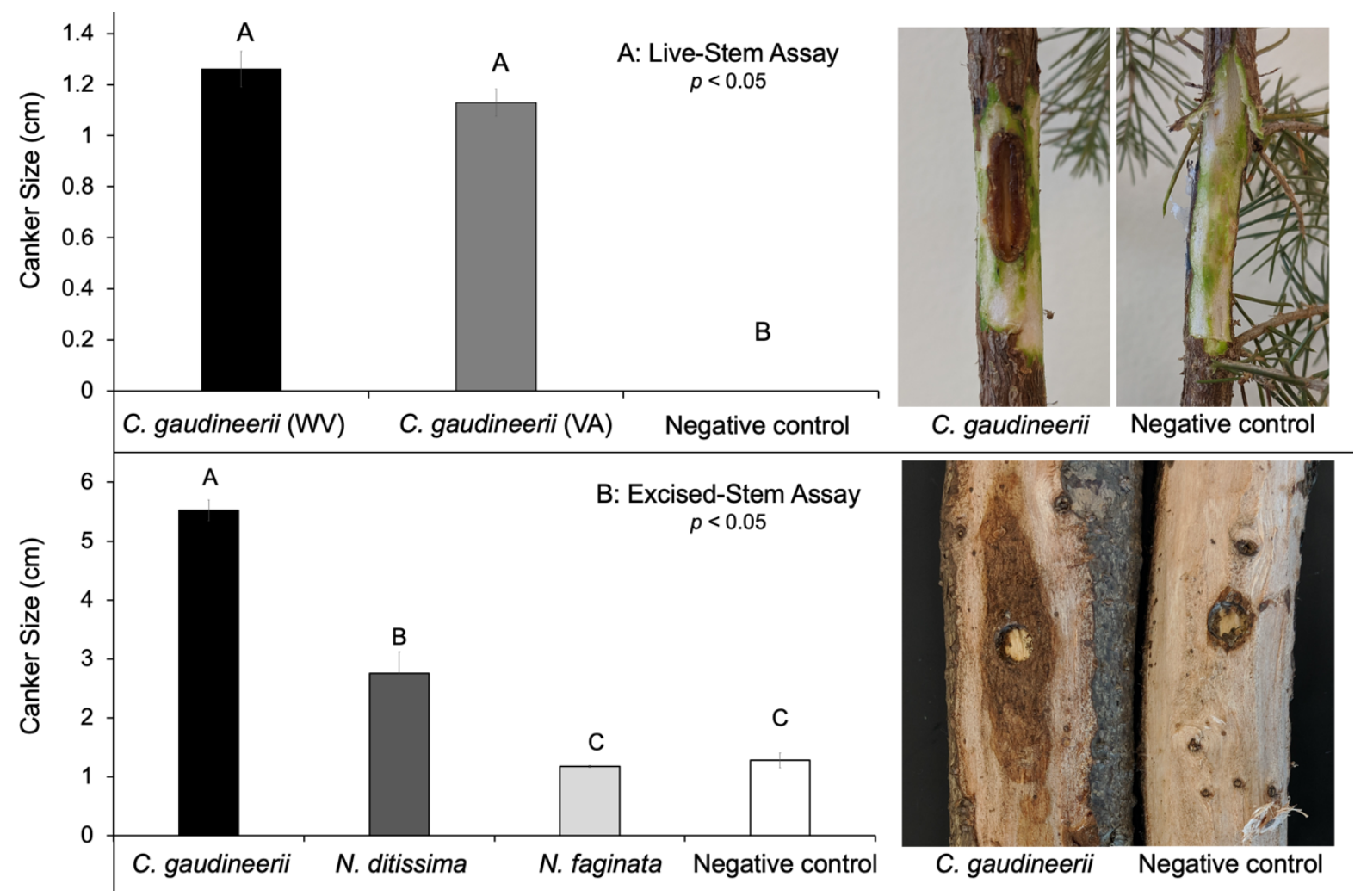

Figure 4: A: Live red spruce stem assay results for inoculations of Corinectria gaudineerii from two separate locations ( $\mathrm{WV}$ and $\mathrm{VA})$ and a negative control. $\mathrm{B}$ : Excised red spruce stem assay for inoculations with C. gaudineerii, Neonectria ditissima, $N$. faginata, and negative control. Letters designate significant differences at $p<0.05$.

\section{Morphological characteristics of C. gaudineerii}

Morphological characteristics of Corinectria gaudineerii sp. nov. are summarized here and illustrated in Figure 5. Perithecia protrude singly or in aggregates (up to >25) through the thin, intact bark tissue of dead red spruce trees and are typically found dispersed along the trunk or possibly larger branches of the tree. Distinct color transitions occur over the life of a perithecium as they typically emerge from thin stroma tissues as smooth, shiny yellowish-orange globose bodies, become bright red with a 
distinct dark-red to black papillate ostiole as they mature, then fade to a dark reddishpurple (Figure 5B). Asci are cylindrical and bear eight ascospores (Figure 5C). Hyaline ascospores $(12.0$ - $18.8 \times 4.6-9.8 \mu \mathrm{m}(\mathrm{avg} .15 .2 \times 7.5 \mu \mathrm{m}))$ are uniseptate with tapered-ends, constricted at the septum, and warty on the surface (Figure D-E). C. gaudineerii cultures have cream-to-buff colored surface with a yellow-to-orange subsurface after $10 \mathrm{~d}$ on PDA (Figure F-G). Microconidial sporodochia are abundantly produced in culture, typically underneath aerial condiophores (Figure $5 \mathrm{H}$ ). Sporodochia are slimy masses with a cream-to-white color. Microconidia (6.4 - 15.7 x $3.3-7.2 \mu \mathrm{m}$ (avg. $9.3 \times 4.9 \mu \mathrm{m}$ ) are hyaline, fusiform, and aseptate (Figure I-K). As with previously described Corinectria spp., C. gaudineerii does not appear to produce macroconidia in culture, although only GYE and PDA media were tested here. 

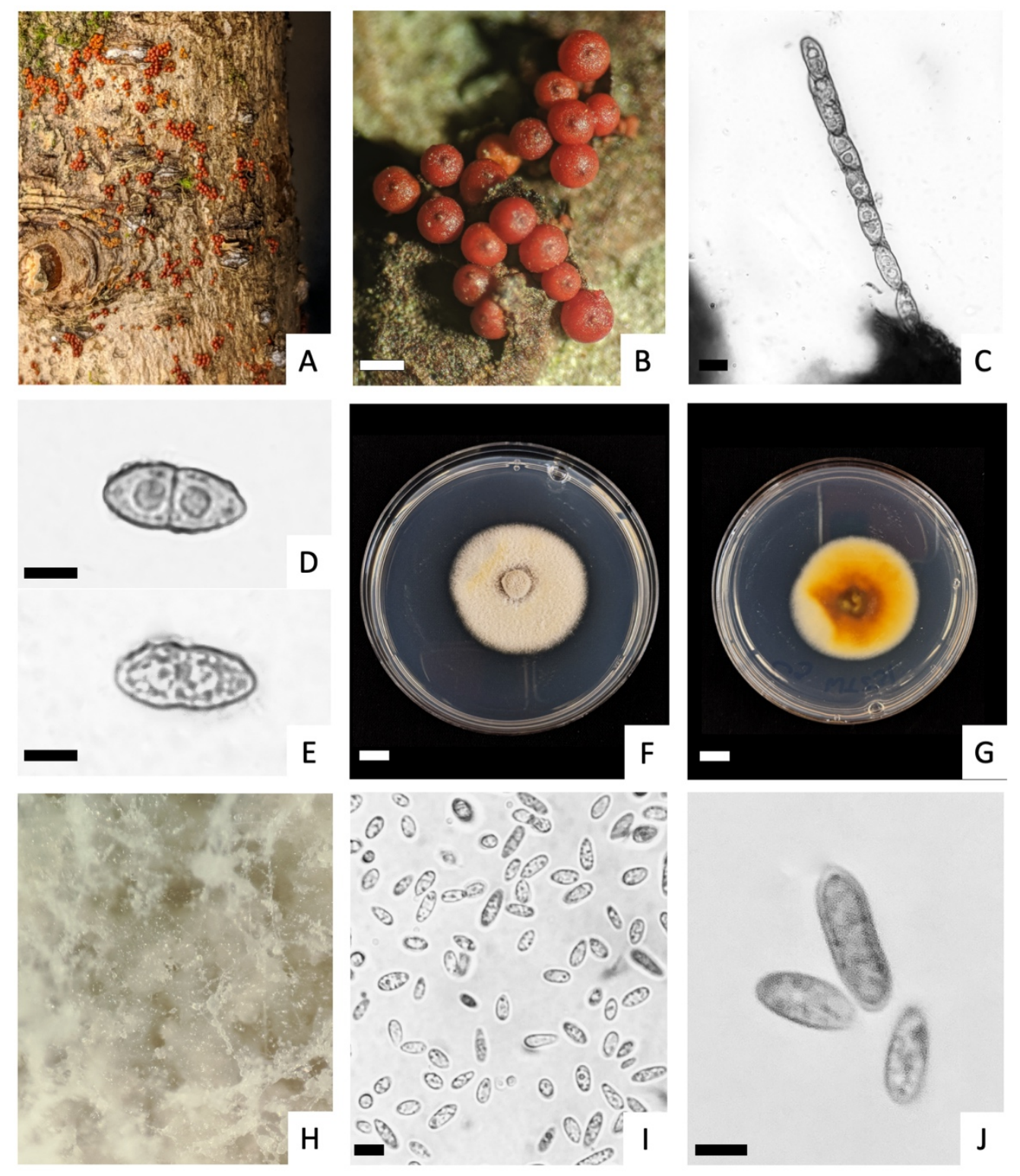

Figure 5: Corinectria gaudineerii: A) Example of abundant fruiting on Picea rubens; B)

C. gaudineerii perithecia erupting from P. rubens bark tissues; C) Ascus containing eight ascospores; D) Ascospore; E) Ornamented surface of ascospore; F) Top of 10day old culture photos on PDA; G) Bottom of culture; H) Microconidial sporodochia underneath aerial conidiophores; I) Microconidia of varying lengths; J) Close-up of microconidia; Scale bars $=250 \mu \mathrm{m}$ for panel $\mathrm{D} ; 20 \mu \mathrm{m}$ for panels $\mathrm{E}, \mathrm{K} ; 10 \mu \mathrm{m}$ for panels F, G, J; $20 \mathrm{~mm}$ for panel $\mathrm{H}$. 


\section{Taxonomy}

Corinectria gaudineerii C. Stauder \& M. Kasson sp. nov.

Etymology: Inspired by the first location C. gaudineerii was observed: Gaudineer Mountain, Monongahela National Forest, Randolph County, West Virginia, USA.

Diagnosis: Differs from C. tsugae by forming perithecia; from C. fuckeliana by forming ascospores with a constricted septum; from C. constricta by producing overlapping ascospores in asci.

Sexual morph: Perithecia formed singly or in clusters of up to 25 or more on thin stromata erumpent through bark tissue of dead red spruce, typically found dispersed along the trunk thus potentially on larger branches too, first yellowish-orange, then bright red, dark reddish-purple when past maturity, globose, forming a papillate, dark red-to-black ostiole, not collapsing with dry. Asci cylindrical bearing eight overlapping ascospores. Ascospores (12.0 - $18.8 \times 4.6$ - $9.8 \mu \mathrm{m}$ (avg. $15.2 \times 7.5 \mu \mathrm{m}))$ uniseptate with tapered-ends, slightly constricted at the septum, hyaline, and warty on the surface.

Asexual morph: Colonies on PDA or GYE cream-to-buff with a yellow-to-orange subsurface after $10 \mathrm{~d}$ at room temperature $\left(\sim 20^{\circ} \mathrm{C}\right)$. Microcondial sporodochia and aerial conidiophores abundant in culture on PDA and GYE media. Microconidia (6.4 $15.7 \times 3.3-7.2 \mu \mathrm{m}$ (avg. $9.3 \times 4.9 \mu \mathrm{m}$ ) hyaline, fusiform, and aseptate. Macroconidia not observed in culture or nature.

Geographic distribution: UNITED STATES: central Appalachian Mountains. 
Habitat: On bark of dead Picea rubens (red spruce) as saprophyte or possibly, hemibiotroph

Type: United States, West Virginia, Gaudineer Knob (38.62928, -79.8429), on dead bark tissue of Picea rubens (red spruce), 28 Dec 2019. M. T. Kasson.

Additional specimens examined: All on bark on dead Picea rubens. United States, Randolph County, Gaudineer Knob, Monongahela National Forest (38.60741, 79.84431), 29 Dec 2018, M. T. Kasson, (GK1, strain CgPr004, strain CgPr007, strain CgPr009). Virginia, Grayson County, Whitetop Mountain, Jefferson National Forest (36.633437, -81.60132), 18 Apr 2019, C. M. Stauder, (WTPIRU4, strain CgPr002). Virginia, Grayson County, Mount Rogers, Jefferson National Forest (36.657625, 81.520676), 19 Apr 2019, C. M. Stauder (MR12, strain CgPr008). West Virginia, Pocahontas County, Cranberry Wilderness, Monongahela National Forest (38.301702, 80.230349), 20 Apr 2019, C. M. Stauder, (CRAN14, strain CgPr010; CRAN32, strain CgPr011).

Notes: C. gaudineerii has morphological features similar to those previously described for C. fuckeliana, C. constricta, and C. tsugae. Cg asci contain overlapping, constricted ascospores which can serve to distinguish it from C. fuckeliana and C. tsugae. Geographic distribution and host range also may be informative for rudimentary identification. Additionally, internal transcribed spacer region (ITS), translation elongation factor 1-alpha (EF1), 28S rDNA (LSU), and actin (ACT) have informative nucleotide substitutions capable of distinguishing this species from C. fuckeliana, C. constricta, and C. tsugae. 


\section{Discussion}

Red spruce experienced several decades of decline, since at least the 1960s (Cook and Zedaker, 1992). Although this decline was eventually associated with anthropogenic activities resulting in acid rain deposition, plant pests and pathogens were initially considered as a major contributor if not the sole cause of this decline. Extensive surveys of declining red spruce stands provided evidence supporting the contribution of these associated pests and pathogens, but not as main causal agents of the decline. In Chapter 2, a novel Corinectria sp. was reported on dead red spruce in central Appalachia, and motivated the investigations included in this study. In the extensive surveys previously conducted in declining red spruce stands a number of fungi associated with dead red spruce were included. Interestingly, none reported the occurrence of any nectriaceous fungi. Therefore, we believe this is the first report of a nectriaceous fungus associated with red spruce in the central Appalachian Mountains.

In this study, we described Corinectria gaudineerii sp. nov. C. Stauder \& M. Kasson through the use of phylogenetic and morphological comparisons among previously described Corinectria spp. Among these data, phylogenetic analyses using concatenated sequence data of the conserved loci BTUB, EF1, ITS, ACT, and LSU support the description of $\mathrm{Cg}$ as a new species (Figure 2A). Additionally, this was supported by nucleotide substitutions in ACT, EF1, ITS, and LSU sequences (Figures 2B,D-F), but BTUB sequences were uninformative as these data did not support the monophyly of any of the previously described Corinectria species (Figure $2 \mathrm{C}$ ). Together, these analyses provide clear evidence that isolates recovered from red spruce in central Appalachia represent a novel species considerably divergent from previously described 
Corinectria sp. As such, $\mathrm{Cg}$ represents the first Corinectria species to be described associated with red spruce in eastern North American forests.

The Corinectria genus was circumscribed as a distinct genus sister to Neonectria (González and Chaverri, 2017). A number of characters can be found to distinguish members of Corinectria from Neonectria (González and Chaverri, 2017). The most obvious of these involves the ecology of these fungi as Corinectria members have only been described from coniferous tree species while nearly all Neonectria host species are solely associated with hardwood tree species. Generally, members of both genera are considered weak pathogens or saprophytes associated with woody tree species. Here, we provide evidence using a live-stem inoculation trial that supports $C g$ to be weakly pathogenic on red spruce (Figure 3 ). This conclusion is made based on the presence of expanded necrosis associated with inoculation sites when compared to the negative control in which no necrotic tissue was observed. The excised stem assay demonstrated the saprophytic capacity of $C g$ on red spruce (Figure 4). Additionally, $\mathrm{Cg}$ was only found on dead red spruce trees with no evidence of symptomology characteristic of canker diseases (e.g. swollen canker margins due to compartmentalization attempts). As such, $\mathrm{Cg}$ would be best described as a hemibiotrophic fungus of red spruce.

In a publication by González and Chaverri (2017), the morphological characters capable of distinguishing among C. constricta, C. fuckeliana, and C. tsugae are provided. The morphology of these species was not directly compared to $C g$ isolates as part of this study, but a few diagnostic differences are highlighted here. First, perithecia of C. tsugae have not been observed (Williams, 1987, González and Chaverri, 2017) 
while the sexual state of $C g$ is dominant on red spruce. In asci of $C$. fuckeliana, ascospores overlap and are unconstricted at the septum, while ascospores in asci of $C$. constricta are constricted and maintain an end-to-end arrangement. Here, $\mathrm{Cg}$ asci appear to contain overlapping, constricted ascospores. Therefore, ascospore arrangement and morphology may serve as a diagnostically informative feature in addition to its apparent ecology and geographic distribution. It is worth noting that the latter represents findings from a limited survey in central Appalachia, and while $\mathrm{Cg}$ was not observed in more northern red spruce forests, the true geographic distribution and host range remains unclear.

Together these results provide evidence supporting Corinectria gaudineerii as a novel hemibiotrophic species on red spruce of central Appalachia. Phylogenetic, morphological, and ecological characteristics capable of discerning this species from previously described Corinectria spp. are also provided. While its pathogenicity on red spruce was not apparent, the fact that this species was not observed in historic surveys of declining red spruce stands is interesting. These surveyed areas may or may not overlap with those included in this study, and as such, expanded surveys are needed to determine the geographic extent of $\mathrm{Cg}$. This is vital to determine the geographic distribution of $\mathrm{Cg}$ within and outside of central Appalachia. Otherwise, the geographic distribution of this species may be greater than that observed in this study. As such, further investigations are warranted to further describe the biology and ecology of $C$. gaudineerii in red spruce forests of eastern North America. 


\section{Literature Cited}

Bruck, R. I. 1989. Survey of diseases and insects of Fraser fir and red spruce in the southern Appalachian Mountains. Eur. J. For. Path. 19: 389-398.

Carbone I., Kohn L.M., 1999. A method for designing primer sets for speciation studies in filamentous fungi. Mycologia 91, 553-556.

Cook, E.R., Zedaker, S.M., 1992. The dendroecology of red spruce decline. In Ecology and decline of red spruce in the eastern United States, Springer, New York, NY.

Crane, P.E., Hopkins, A.J., Dick, M.A. and Bulman, L.S., 2009. Behaviour of Neonectria fuckeliana causing a pine canker disease in New Zealand. Canadian Journal of Forest Research, 39, 2119-2128.

Dick, M.A. and Crane, P.E., 2009. Neonectria fuckeliana is pathogenic to Pinus radiata in New Zealand. Australasian Plant Disease Notes, 4, 12-14.

Gadgil, P.D., Dick, M.A. and Dobbie, K., 2003. Fungi silvicolae novazelandiae: 4. New Zealand Journal of Forestry Science 33, 265-272.

González, C.D. and Chaverri, P., 2017. Corinectria, a new genus to accommodate Neonectria fuckeliana and C. constricta sp. nov. from Pinus radiata in Chile. Mycological Progress, 16(11-12), pp.1015-1027.

Huse, K.J., 1981. The distribution of fungi in sound-looking stems of Picea abies in Norway. European Journal of Forest Pathology, 11, 1-6.

Johnson, A. H., S. B. McLaughlin. 1986. The nature and timing of the deterioration of red spruce in the northern Appalachians. Acid Deposition Long-Term Trends, National Academy of Sciences Press, p. 200. 
Katoh K., Standley D.M., 2013. MAFFT multiple sequence alignment software version 7: improvements in performance and usability. Mol. Biol. Evol. 30, 772-780.

Landan G., Graur D., 2008. Local reliability measures from sets of co-optimal multiple sequence alignments. Pacific Symposium on Biocomputing 13, 15-24.

Macias, A.M., Geiser, D.M., Stajich, J.E., Lukasik, P., Veloso, C., Bublitz, D.C., Berger, M.C., Boyce, G.R., Hodge, K. and Kasson, M.T., 2019. Evolutionary relationships among Massospora spp.(Entomophthorales), obligate pathogens of cicadas. Mycologia.

O'Donnell, K., Cigelnik, E., 1997. Two divergent intragenomic rDNA ITS2 types within a monophyletic lineage of the fungus Fusarium are nonorthologous. Mol. Phylogenet. Evol. 7, 103-116.

Prasad, A. M., Iverson, L.R., 2003. Little's range and FIA importance value database for 135 eastern US tree species. Northeastern Research Station, USDA Forest Service, Delaware, Ohio.

R Core Team., 2020. R: a language and environment for statistical computing. $R$ Foundation for Statistical Computing, Vienna, Austria.

Rambaut, A., 2017. FigTree-version 1.4.3, a graphical viewer of phylogenetic trees.

Rambaut, A., Drummond, A.J., Xie, D., Baele, G., Suchard, M.A., 2018. Posterior summarization in Bayesian phylogenetics using Tracer 1.7. Syst. Biol. 67, 901904.

Rehner, S., 2001. Primers for Elongation Factor 1- $\alpha$ (EF1- $\alpha)$. Insect Biocontrol Laboratory: USDA, ARS, PSI.

Rehner, S.A., Samuels, G.J., 1994. Taxonomy and phylogeny of Gliocladium analyzed 
from nuclear large subunit ribosomal DNA sequences. Mycol. Res. 98, 625-634.

Roll-Hansen, F. and Roll-Hansen, H., 1979. Microflora of sound-looking wood in Picea abies stems. European Journal of Forest Pathology 9, 308-316.

Ronquist, F., Teslenko, M., Van Der Mark, P., Ayres, D.L., Darling, A., Höhna, S., Larget, B., Liu, L., Suchard, M.A., Huelsenbeck, J.P., 2012. MrBayes 3.2: efficient Bayesian phylogenetic inference and model choice across a large model space. Syst. Biol. 61, 539-542.

Sela, I., Ashkenazy, H., Katoh, K., Pupko, T., 2015. GUIDANCE2: accurate detection of unreliable alignment regions accounting for the uncertainty of multiple parameters. Nucleic Acids Res., 43, W7-W14.

Stecher, G., Tamura, K., Kumar, S., 2020. Molecular Evolutionary Genetics Analysis (MEGA) for macOS. Mol. Biol. Evol.

Vasiliauskas, R. and Stenlid, J., 1998. Fungi inhabiting stems of Picea abies in a managed stand in Lithuania. Forest ecology and management 109, 119-126.

Vilgalys, R., Hester, M., 1990. Rapid genetic identification and mapping of enzymatically amplified ribosomal DNA from several Cryptococcus species. J. Bacteriol. 172, 4238-4246.

Villesen, P., 2007. FaBox: an online toolbox for fasta sequences. Mol. Ecol. Notes, 7, 965-968.

White, T.J., Bruns, T., Lee, S., Taylor, J. 1990. Amplification and direct sequencing of fungal ribosomal RNA genes for phylogenetics. PCR Protocol. Guide Methods. Appl. 18, 315-322.

Williams, M.A.J., 1987. Acremonium tsugae. Mycopathologia 100, 177-178. 


\section{Supplemental Tables}

Supplemental Table 1: Locations of survey sites. The presence of Corinectria gaudineerii is noted for each site. Additionally, the general coordinates of the sampling locations are provided. Samples were only collected within permitted areas.

\begin{tabular}{lccc}
\hline Site & Location & BBD Present & Coordinates \\
\hline MR & Grayson County, VA & Y & $(36.657625,-81.520676)$ \\
WT & Grayson County, VA & Y & $(36.633437,-81.60132)$ \\
GK & Randolph County, WV & Y & $(38.60741,-79.84431)$ \\
CR & Pocahontas County, WV & Y & $(38.301702,-80.230349)$ \\
DS & Tucker County, WV & N & $(39.015311,-79.343767)$ \\
CS & Preston County WV & N & $(39.537414,-79.482550)$ \\
SK & Pendleton County, WV & N & $(38.699077,-79.533215)$ \\
\hline
\end{tabular}


Supplemental Table 2: Primer sequences, PCR protocols, and sources for each primer pair used to generate sequence data within this study.

\begin{tabular}{|c|c|c|c|c|c|c|c|c|c|c|c|c|c|c|}
\hline $\begin{array}{l}\text { Target } \\
\text { Locus }\end{array}$ & Primer & Primer Sequence & \multicolumn{2}{|c|}{$\begin{array}{c}\text { Initial } \\
\text { Denature }\end{array}$} & $\begin{array}{l}\text { Cycle } \\
\text { s }\end{array}$ & \multicolumn{2}{|c|}{ Denature } & \multicolumn{2}{|c|}{ Anneal } & \multicolumn{2}{|c|}{ Extension } & \multicolumn{2}{|c|}{$\begin{array}{c}\text { Final } \\
\text { Extension }\end{array}$} & Source \\
\hline ITS & ITS5 & $\begin{array}{c}\text { GGAAGTAAAAGTCGTAACAAG } \\
\text { G } \\
\text { TCCTCCGCTTATTGATATGC }\end{array}$ & $95 \mathrm{C}$ & $\begin{array}{c}2: 0 \\
0\end{array}$ & 35 & $\begin{array}{c}95 \\
\mathrm{C}\end{array}$ & $\begin{array}{c}0: 3 \\
0\end{array}$ & $56 C$ & $\begin{array}{c}0: 3 \\
0\end{array}$ & $\begin{array}{l}72 \\
C\end{array}$ & $\begin{array}{c}1: 0 \\
0\end{array}$ & $72 \mathrm{C}$ & $\begin{array}{c}7: 0 \\
0\end{array}$ & $\begin{array}{l}\text { White et al., } \\
1990\end{array}$ \\
\hline \multirow{2}{*}{ tef-1 $1 \alpha$} & $\begin{array}{l}\text { EF1- } \\
728\end{array}$ & CATCGAGAAGTTCGAGAAGG & \multirow{2}{*}{$95 \mathrm{C}$} & \multirow{2}{*}{$\begin{array}{c}2: 0 \\
0\end{array}$} & \multirow{2}{*}{35} & \multirow{2}{*}{$\begin{array}{l}95 \\
\mathrm{C}\end{array}$} & \multirow{2}{*}{$\begin{array}{c}0: 3 \\
0\end{array}$} & \multirow{2}{*}{$56 \mathrm{C}$} & \multirow{2}{*}{$\begin{array}{c}0: 3 \\
0\end{array}$} & \multirow{2}{*}{$\begin{array}{l}72 \\
C\end{array}$} & \multirow{2}{*}{$\begin{array}{c}1: 0 \\
0\end{array}$} & \multirow{2}{*}{$72 \mathrm{C}$} & \multirow{2}{*}{$\begin{array}{c}7: 0 \\
0\end{array}$} & $\begin{array}{l}\text { Carbone and } \\
\text { Kohn, } 1999\end{array}$ \\
\hline & $\begin{array}{l}\text { EF1- } \\
1576\end{array}$ & $\begin{array}{c}\text { ACHGTRCCRATACCACCRATC } \\
\text { TT }\end{array}$ & & & & & & & & & & & & Rehner 2001 \\
\hline \multirow{2}{*}{ nrLSU } & LR5 & ATCCTGAGGGAAACTTC & \multirow{2}{*}{$95 \mathrm{C}$} & \multirow{2}{*}{$\begin{array}{c}2: 0 \\
0\end{array}$} & \multirow{2}{*}{35} & \multirow{2}{*}{$\begin{array}{l}95 \\
\mathrm{C}\end{array}$} & \multirow{2}{*}{$\begin{array}{c}0: 3 \\
0\end{array}$} & \multirow{2}{*}{$\begin{array}{c}51.1 \\
C\end{array}$} & $0: 4$ & 72 & $1: 3$ & $72 \mathrm{C}$ & 7:0 & $\begin{array}{l}\text { Vilgalys and } \\
\text { Hester, } 1990 \\
\end{array}$ \\
\hline & LROR & GTACCCGCTGAACTTAAGC & & & & & & & 5 & $C$ & 0 & $1<0$ & 0 & $\begin{array}{c}\text { Rehner and } \\
\text { Samuels, } 1994\end{array}$ \\
\hline & Tact1 & $\begin{array}{c}\text { TGGCACCACACCTTCTACAAT } \\
\text { GA }\end{array}$ & & $2: 0$ & & & $0: 3$ & & & & 1:0 & & $7: 0$ & Samuels et al., \\
\hline ACIIN & $\begin{array}{c}\text { Tact2 } \\
1 \\
\end{array}$ & $\begin{array}{c}\text { TCTCCTTCTGCATACGGTCGG } \\
\text { A }\end{array}$ & $95 \mathrm{C}$ & 0 & 35 & $\mathrm{C}$ & 0 & $55 \mathrm{C}$ & 5 & $\mathrm{C}$ & 0 & $12 \mathrm{C}$ & 0 & 2006 \\
\hline Beta- & T1 & AACATGCGTGAGATTGTAAGT & & $2: 0$ & & 95 & $0: 3$ & & $0: 3$ & 72 & $1: 0$ & & $7: 0$ & O'Donnell and \\
\hline tublin & $\mathrm{T} 2$ & $\begin{array}{c}\text { TAGTGACCCTTGGCCCAGTT } \\
\text { G- }\end{array}$ & $95 \mathrm{C}$ & 0 & 35 & $\mathrm{C}$ & 0 & $64 C$ & 0 & $\mathrm{C}$ & 0 & $72 \mathrm{C}$ & 0 & Cigelnik, 1997 \\
\hline
\end{tabular}

